\title{
Estudio del desplazamiento lateral de un decelerador de alas rotatorias de pequeño alargamiento a través de las variaciones cíclicas del paso de las palas
}

Tesis doctoral presentada por

\section{Juan Francisco Martiarena}

\author{
ante la \\ Facultad de Ingeniería de la \\ Universidad Nacional de La Plata \\ para acceder al grado académico de
}

DOCTOR EN INGENIERÍA

Dirección de la tesis:

Vicente Nadal Mora, Joaquín Piechocki, Álvaro Cuerva Tejero.

La Plata, 27 de Marzo de 2018 


\section{Índice}

1. Resumen 1

2. Introducción 3

2.1. Introducción al concepto de pararrotor . . . . . . . . . . . . . . 3

2.2. Antecedentes bibliográficos . . . . . . . . . . . . . . . 3

2.2.1. Antecedentes bibliográficos en aerodinámica de bajo número de Reynolds y pequeño alargamiento . . . . . . . . . . . . 3

2.2.2. Antecedentes bibliográficos sobre casos naturales . . . . . . . 5

2.2.3. Antecedentes bibliográficos sobre casos de aplicación tecnológica 7

2.3. Aplicaciones . . . . . . . . . . . . . . . . . . . . . 12

3. Estudio teórico del desplazamiento lateral de un decelerador aerodinámico a través de las variaciones cíclicas del paso de las palas 13

3.1. Introducción . . . . . . . . . . . . . . . . . . . . . . 13

3.2. Autorrotación . . . . . . . . . . . . . . . . . . . 14

3.3. Definiciones generales del modelo teórico . . . . . . . . . . . . . . 15

3.4. Modelo dinámico del pararrotor en vuelo libre . . . . . . . . . . . . . 19

3.5. Fuerzas sobre el cilindro . . . . . . . . . . . . . . . . . . . . 20

3.6. Ligadura cinemática . . . . . . . . . . . . . . . . . . . 23

3.7. Ecuaciones de la trayectoria del centro de masas del cuerpo del pararrotor ........................... 24

3.8. Fuerzas sobre las palas . . . . . . . . . . . . . . . . . . . 25

3.8.1. Leyes de variación de paso . . . . . . . . . . . . . . . 28

3.9. Forma canónica de la ecuación de estado del pararrotor . . . . . . . . 28

3.9.1. Forma canónica de la ecuación de Newton . . . . . . . . . . . 30

3.9.2. Forma canónica de la ecuación de Euler . . . . . . . . . . . . . 31

3.9.3. Forma canónica de la ligadura cinemática y la ecuación de trayectoria . . . . . . . . . . . . . . . 31

3.9.4. Ecuación de estado en forma canónica . . . . . . . . . . . . 32

3.10. Solución de la ecuación de estado del pararrotor . . . . . . . . . . . 33

3.10.1. Cálculo de fuerzas y momentos de origen inercial . . . . . . . 33

3.10.2. Cálculo de fuerzas y momentos de origen aerodinámico . . . . 36 
3.10.3. Resolución del sistema de ecuaciones . . . . . . . . . . . . . . 40

3.11. Modelo del pararrotor en túnel de viento . . . . . . . . . . . . . . . . 41

3.11.1. Desarrollo en serie de Taylor del modelo en túnel de viento . . 45

3.11.2. Solución del modelo en series de Taylor del pararrotor en túnel de viento . . . . . . . . . . . . . . . . . . . 49

3.11.3. Otros resultados derivados de la resolución del modelo teórico 55 3.12. Conclusiones del capítulo . . . . . . . . . . . . . . . . 58

4. Estudio experimental del desplazamiento lateral de decelerador aerodinámico a través de las variaciones cíclicas del paso de las palas 61

4.1. Introducción . . . . . . . . . . . . . . . . . . . . . . 61

4.2. Descripción del modelo físico . . . . . . . . . . . . . . . . . . 61

4.3. Configuración experimental . . . . . . . . . . . . . 63

4.3.1. Análisis estático de la bancada . . . . . . . . . . . . . 65

4.4. Parámetros medidos e instrumentación . . . . . . . . . . . . . . . 67

4.4.1. Medición de la fuerza en la balanza . . . . . . . . . . . . . . 68

4.5. Control del paso cíclico de las palas . . . . . . . . . . . . . . . . . . 69

4.6. Ensayos . . . . . . . . . . . . . . . . . . . 71

4.6.1. Medición de la fuerza en la balanza . . . . . . . . . . . . . 71

4.6.2. Resultados . . . . . . . . . . . . . . . . . . 72

4.7. Incertidumbre de las mediciones . . . . . . . . . . . . . . . . . . 77

4.8. Conclusiones del capítulo . . . . . . . . . . . . . . . . . . . . 82

5. Simulaciones de trayectorias del desplazamiento de un decelerador aerodinámico a través de las variaciones cíclicas del paso de las palas $\quad 83$

5.1. Introducción . . . . . . . . . . . . . . . . . . . . . . . 83

5.2. Modelo matemático . . . . . . . . . . . . . . . . . . . . . 83

5.3. Vuelo equilibrado del pararrotor . . . . . . . . . . . . . . . . 84

5.3.1. Modelado de las acciones transmitidas al pararrotor . . . . . . 85

5.3.2. Forma canónica de la ecuación de equilibrio . . . . . . . . . 86

5.3.3. Características de la solución del equilibrado . . . . . . . . . . 88

5.3.4. Solución del problema dinámico completo a partir de leyes de control obtenidas en la solución del equilibrio . . . . . . . . . 92

5.4. Conclusiones del capítulo . . . . . . . . . . . . . . . . . . . . . 101

6. Conclusiones 102

6.1. Introducción . . . . . . . . . . . . . . . . . . . . 102

6.1.1. Modelo teórico . . . . . . . . . . . . . . . 102

6.1.2. Modelo experimental . . . . . . . . . . . . . . . 103

6.1.3. Simulación de trayectorias . . . . . . . . . . . . . . . . 104

6.1.4. Futuras líneas de investigación . . . . . . . . . . . . . . . 104

6.2. Aportes originales de la tesis . . . . . . . . . . . . . . . . 106 
7. Anexos

7.1. Caracterización aerodinámica de las palas . . . . . . . . . . . . 107

7.2. Obtención de expresiones matemáticas para el desarrollo en serie de

Taylor del modelo en túnel de viento . . . . . . . . . . . . . . . . . 110

7.2.1. Obtención de términos inerciales . . . . . . . . . . . . . . . 110

7.2.2. Obtención de términos aerodinámicos . . . . . . . . . . . . . 114

7.2.3. Obtención de términos gravitatorios . . . . . . . . . . 116 


\section{Lista de Figuras}

1. Semilla y modelo artificial . . . . . . . . . . . . . . . . . 7

2. Modelo de pararrotor en túnel vertical . . . . . . . . . . . . . . . . . 9

3. Recuperadores de sondas . . . . . . . . . . . . . . . . . . . 11

4. Ángulos de Euler . . . . . . . . . . . . . . . . . . . . . . . 15

5. Ángulos de Euler . . . . . . . . . . . . . . . . . . . . . . 17

6. Ángulos de Euler . . . . . . . . . . . . . . . . . . . . . . . 17

7. Referencias geométricas . . . . . . . . . . . . . . . . 18

8. Fuerzas de la pala sobre el cilindro . . . . . . . . . . . . . . . 21

9. Fuerzas y momentos sobre el cilindro . . . . . . . . . . . . . . . 21

10. Fuerzas sobre la pala . . . . . . . . . . . . . . . . 25

11. Fuerzas sobre la pala . . . . . . . . . . . . . . . . . . . . 26

12. Momento inercial . . . . . . . . . . . . . . . . . . 34

13. Acciones inerciales . . . . . . . . . . . . . . . . 34

14. Diagrama de fuerzas sobre la pala . . . . . . . . . . . . . . . 36

15. Diagrama de fuerzas sobre la pala vista en planta . . . . . . . . . 37

16. Esquema de montaje . . . . . . . . . . . . . . . . . . . 41

17. Esquema de transmisión de fuerzas y momentos . . . . . . . . . . . . . . 43

18. Velocidad angular adimensional . . . . . . . . . . . . . . . 52

19. Velocidad angular . . . . . . . . . . . . . . . . . . 53

20. Velocidad angular dimensional . . . . . . . . . . . . . . . 53

21. Velocidad angular . . . . . . . . . . . . . . . . . . . 54

22. Velocidad angular dimensional . . . . . . . . . . . . . . . . 54

23. Momento aerodinámico para distintos valores del paso cíclico longitudinal . . . . . . . . . . . . . . . . 56

24. Momento inercial para distintos valores del paso cíclico longitudinal . 56

25. Momento respecto al eje de la pala . . . . . . . . . . . . . . . . . 58

26. Ángulo de ataque de la pala . . . . . . . . . . . . . . . . . 58

27. Dimensiones del modelo . . . . . . . . . . . . . . . . . . . 62

28. Esquema del soporte . . . . . . . . . . . . . . . . . . . . . . 64

29. Modelo en túnel . . . . . . . . . . . . . . . . . . . . . . . . . 64

30. Puntos de referencia sobre el pararrotor . . . . . . . . . . . . . . 65

31. Esquema de modelo experimental . . . . . . . . . . . . . . . . . . 66

32. Esquema de la celda . . . . . . . . . . . . . . . . . . . . . . . 69 
33. Interfaz del sistema de adquisición . . . . . . . . . . . . . . . 70

34. Puntos de calibración . . . . . . . . . . . . . . . . . . 71

35. Medida de la balanza . . . . . . . . . . . . . . . . . . . . . 75

36. Fuerza en la balanza . . . . . . . . . . . . . . . . . 76

37. Fuerza en la balanza . . . . . . . . . . . . . . . . . . . 77

38. Comparación teórico-experimental . . . . . . . . . . . . . 78

39. Comparación teórico-experimental . . . . . . . . . . . . . . . 78

40. Comparación teórico-experimental . . . . . . . . . . . . . . . . 79

41. Comparación teórico-experimental . . . . . . . . . . . . . . . . . 79

42. Comparación teórico-experimental . . . . . . . . . . . . . . . . . . . . . . . . . . 80

43. Comparación teórico-experimental . . . . . . . . . . . . . . 80

44. Fuerzas en vuelo . . . . . . . . . . . . . . . . . . . . . 85

45. Diagrama de velocidades y ángulos auxiliares . . . . . . . . . . . . . . . . . 88

46. Soluciones del sistema equilibrado . . . . . . . . . . . . . . . . . . . . . 90

47. Ángulo de paso equilibrado . . . . . . . . . . . . . . . . . . . . . . . . . . . . . . 92

48. Soluciones del sistema canónico . . . . . . . . . . . . . . . . . 95

49. Soluciones del sistema equilibrado . . . . . . . . . . . . . . 96

50. Soluciones del sistema equilibrado . . . . . . . . . . . . . . . . . . . . . 97

51. Variables cinemáticas según trayectoria . . . . . . . . . . . . . . . . 100

52. Coeficiente de presiones . . . . . . . . . . . . . . . 108

53. Coeficiente de resistencia . . . . . . . . . . . . . . . 109

\section{Lista de Cuadros}

1. Parámetros generales para la resolución del sistema (84) . . . . . . . 51

2. Parámetros generales para la resolución del sistema (84) . . . . . . . 52

3. Dimensiones de las palas . . . . . . . . . . . . . . . . . . . . 62

4. Instrumentos utilizados . . . . . . . . . . . . . . . . . . . 68

5. Datos técnicos celda de carga . . . . . . . . . . . . . . 68

6. Datos técnicos de los servomecanismos . . . . . . . . . . . . . . 69

7. Ángulos de paso cíclico para la calibración . . . . . . . . . . . . . . 71

8. Ángulos de ensayo . . . . . . . . . . . . . . . . . . . 72

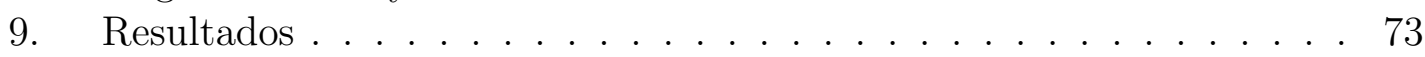

10. Comparación de resultados . . . . . . . . . . . . . . . . . 73 
11. Resultados . . . . . . . . . . . . . . . . . . . 74

12. Incertidumbre combinada $\ldots \ldots \ldots \ldots$. . . . . . . . 81

13. Elementos prefijados del vector de estado . . . . . . . . . . . . . . 89

14. Fuerzas y momentos de equilibrio para $\beta_{T}=0, w_{T}=8 \mathrm{~m} / \mathrm{s}$ y $V_{H}=3 \mathrm{~m} / \mathrm{s} 93$

15. Elementos prefijados del vector de estado . . . . . . . . . . . 93

16. Elementos prefijados del vector de estado . . . . . . . . . . . . . . 98

17. Órdenes de magnitud . . . . . . . . . . . . . . . . . . . . 114

18. Órdenes de magnitud . . . . . . . . . . . . . . . . . 116 


\section{Lista de Símbolos}

\section{Símbolos Latinos}

$A(\psi)$ Coeficiente del término cuadrático de la ecuación diferencial del modelo en túnel de viento

A Punto de intersección entre el eje de las palas y el eje del cilindro del pararrotor

$\mathbf{A E}_{b} \quad$ Vector que une el punto A con el punto $\mathrm{E}_{b}$

$\mathbf{a}^{G} \quad$ Vector aceleración del centro de masas del pararrotor

$\mathbf{a}_{b}^{G B} \quad$ Vector aceleración del centro de masas de la pala $b$

$A_{b} \quad$ Área barrida por el pararrotor

$a_{r} \quad$ coeficiente de ajuste de datos experimentales

$A B S$ Acrilonitrilo Butadieno Estireno

AR Alargamiento de la pala, $\left(\frac{b}{c}\right)$

$B(\psi)$ Coeficiente del término lineal de la ecuación diferencial del modelo en túnel de viento

b $\quad$ Envergadura de la pala

$b \quad$ número de palas del pararrotor

$C(\psi)$ Coeficiente del término independiente de la ecuación diferencial del modelo en túnel de viento 
c Cuerda de la pala

$C_{1} \quad$ Punto de acoplamiento entre la barra y la varilla de la celda de carga

$C_{D_{H}} \quad$ Coeficiente de resistencia del cilindro

$C_{D} \quad$ Coeficiente de resistencia de la pala

$C_{L \alpha}$ pendiente del coeficiente de sustentación de la pala

$C_{l \alpha} \quad$ pendiente del coeficiente de sustentación de la sección

$C_{L} \quad$ Coeficiente de sustentación de la pala

$C_{p}(x)$ Posición del centro de presiones

d parámetro adimensional de momentos de inercia

$D_{b} \quad$ resistencia aerodinámica de la pala $b$

$e \quad$ excentricidad del punto de acople $E_{b}$ respecto al centro de masas del pararrotor

$\mathbf{E}_{b} \mathbf{G B}_{b}$ Vector que une el punto $E_{b}$ con el punto $G B_{b}$

$\mathbf{E}_{b} \mathbf{P}_{b}$ Vector que une el punto $E_{b}$ con el punto $P_{b}$

$E_{b} \quad$ Punto de acoplamiento de la pala al pararrotor

F $\quad$ Fuerza teórica medida en la celda de carga

F Punto de aplicación de fuerzas y momentos aerodinámicos del cilindro del pararrotor

f Vector de funciones del sistema diferencial

$\mathbf{F}_{b}^{a} \quad$ Fuerza aerodinámica de la pala $b$

$\mathbf{F}_{H}^{g} \quad$ Fuerza gravitatoria del cilindro del pararrotor

$\mathbf{F}_{H}^{i} \quad$ Fuerza inercial del cilindro del pararrotor 
$\mathbf{F}_{b}^{r} \quad$ Fuerza de reacción sobre la pala $b$

$\mathbf{F}^{t} \quad$ Fuerza transmitida por las palas al cilindro

$\mathbf{F}_{b}^{t} \quad$ Fuerza transmitida por la pala $b$ al cilindro

$\mathbf{F}^{r, O} \quad$ Fuerza de reacción sobre el pararrotor en el punto $\mathrm{O}$

$\mathbf{F}_{H}^{a} \quad$ Fuerza aerodinámica sobre el cilindro del pararrotor

$F_{x_{A}}^{\exp } \quad$ Fuerza experimental medida en la celda de carga

$F_{i_{j}}^{a} \quad$ Componente $i_{j}$ de la fuerza aerodinámica de las palas, $i=\mathrm{x}, \mathrm{y}, \mathrm{z} ; j=\mathrm{A}, \mathrm{A} 1, \mathrm{~B}$

G Centro de masas del cilindro del pararrotor

$g \quad$ aceleración de la gravedad

$G S \quad$ Centro de masas de la barra del sistema experimental

g Vector aceleración de la gravedad

GA Vector que une el punto $\mathrm{G}$ con el punto A

$\mathbf{G E}_{b} \quad$ Vector que une el punto $\mathrm{G}$ con el punto $\mathrm{E}_{b}$

GF Vector que une el punto $\mathrm{G}$ con el punto $\mathrm{F}$

$G B_{b} \quad$ Centro de masas de la pala $b$

H Operador hessiano

$h \quad$ relación entre $I_{H}$ y $I_{z_{B}}$

$\mathbf{h}_{B_{b}} \quad$ Momento cinético de la pala $b$ en ejes B

$\mathbf{h}_{H} \quad$ Momento cinético del cuerpo del pararrotor

$\mathbf{I}_{B} \quad$ Tensor de inercia de la pala en ejes B

$I \mathbf{I}_{H} \quad$ Tensor de inercia del cuerpo del pararrotor 
$I_{3} \quad$ Momento de inercia del cuerpo del pararrotor en la dirección $\mathrm{z}_{A}$

$K \quad$ factor de resistencia inducida

$K_{p} \quad$ parámetro variable de ajuste del coeficiente de sustentación

$K_{v} \quad$ parámetro constante de ajuste del coeficiente de sustentación

L Distancia entre los puntos G y A

LC Matriz de ligadura cinemática

$L_{b} \quad$ sustentación aerodinámica de la pala $b$

$l_{0} \quad$ Distancia entre los puntos GS y O

$l_{C_{1}} \quad$ Distancia entre los puntos $\mathrm{C}$ y $\mathrm{C}_{1}$

$l_{i_{b}} \quad$ dimensión genérica de una pala, con $i=\mathrm{x}, \mathrm{y}, \mathrm{z}$.

$l_{O A} \quad$ Distancia entre los puntos $\mathrm{O}$ y $\mathrm{A}$

M parámetro de adimensionalización

M Matriz de masas del sistema diferencial

$\mathbf{M}^{r, O}$ Momento de reacción sobre el pararrotor en el punto $\mathrm{O}$

$\mathbf{M}^{t, A}$ Momento transmitido por las palas al cilindro en el punto A

$\mathbf{M}^{t, E_{b}}$ Momento transmitido por la pala $b$ al cilindro en el punto $\mathrm{E}_{b}$

$\mathbf{M}^{t, G}$ Momento transmitido por las palas al cilindro en el punto $\mathrm{G}$

$\mathbf{M}_{H}^{a, F}$ Momento aerodinámico sobre el cilindro del pararrotor en el punto $\mathrm{F}$

$\mathbf{M}_{H}^{a, G}$ Momento aerodinámico sobre el cilindro del pararrotor en el punto $\mathrm{G}$

$\mathbf{M}_{H}^{i, G}$ Momento inercial sobre el cilindro del pararrotor en el punto $\mathrm{G}$

$M_{S} \quad$ Masa de la barra del sistema experimental 
$M_{b, i_{j}}^{r, E_{b}}$ Componente $i_{j}$ del momento de reacción sobre una pala en el punto $E_{b}$, $i=\mathrm{x}, \mathrm{y}, \mathrm{z} ; j=\mathrm{A}, \mathrm{A} 1, \mathrm{~B}$

$M_{b, i_{j}}^{t, E_{b}}$ Componente $i_{j}$ del momento transmitido de una pala en el punto $E_{b}, i=\mathrm{x}, \mathrm{y}, \mathrm{z}$; $j=\mathrm{A}, \mathrm{A} 1, \mathrm{~B}$

$M_{b} \quad$ masa de la pala

$M_{H} \quad$ masa del cuerpo del pararrotor

$M_{i_{j}}^{a, A}$ Componente $i_{j}$ del momento aerodinámico de las palas en el punto A, $i=\mathrm{x}, \mathrm{y}, \mathrm{z}$; $j=\mathrm{A}, \mathrm{A} 1, \mathrm{~B}$

$M_{i_{j}}^{a, E_{b}}$ Componente $i_{j}$ del momento aerodinámico de una pala en el punto $E_{b}$, $i=\mathrm{x}, \mathrm{y}, \mathrm{z} ; j=\mathrm{A}, \mathrm{A} 1, \mathrm{~B}$

n Parámetro dependiente del alargamiento

NASA National Aeronautics and Space Administration

$P_{b} \quad$ Punto de aplicación de la fuerza aerodinámica de la pala $b$

$P_{a t m}$ Presión atmosférica medida en el ensayo

Q Presión dinámica

$\mathbf{r}^{G} \quad$ Vector posición del centro de masas del pararrotor

$R_{b} \quad$ radio de referencia para la adimensionalización de coeficientes

$r_{G_{i}} \quad$ coordenada $G_{i}$ del centro de masas del pararrotor en ejes tierra, con $i=1,2,3$.

$S \quad$ Superficie de la pala

$S_{H} \quad$ Superficie de referencia del cilindro

$\mathbf{T}_{A 1 b B_{b}}$ Matriz de transformación del sistema B al sistema A1 de la pala $b$

$\mathbf{T}_{A T 1}$ Matriz de transformación del sistema T1 al sistema A

$\mathbf{T}_{B_{b} A 1 b}$ Matriz de transformación del sistema $\mathrm{A} 1 \mathrm{~b}$ al sistema $\mathrm{Bb}$ 
$\mathbf{T}_{B_{b} W 1 b}$ Matriz de transformación del sistema W1b al sistema Bb

$\mathbf{T}_{T 1 T}$ Matriz de transformación del sistema $\mathrm{T}$ al sistema T1

$\mathbf{T}_{W 1 b W b}$ Matriz de transformación del sistema Wb al sistema W1b

$T_{b h} \quad$ Temperatura de bulbo húmedo medida en el ensayo

$T_{b s} \quad$ Temperatura de bulbo seco medida en el ensayo

$T_{i} \quad$ Punto de articulación de la barra del sistema experimental, donde $i$ indica superior o inferior

$t_{s} \quad$ Tiempo de simulación para el sistema dinámico completo

$u \quad$ Velocidad del centro de masas del pararrotor en la dirección $x_{A}$

$\mathbf{u}_{C}(t)$ Vector de control

$\mathbf{U}_{W} \quad$ Velocidad del viento respecto al pararrotor

$U_{0} \quad$ Velocidad de referencia para la adimensionalización de coeficientes

$U_{F_{x}^{e x p}}^{e x}$ Incertidumbre combinada del momento experimental

$u_{T} \quad$ Velocidad del centro de masas del pararrotor en la dirección $x_{T}$

$v \quad$ Velocidad del centro de masas del pararrotor en la dirección $y_{A}$

$\mathbf{V}^{G} \quad$ Vector velocidad del centro de masas del pararrotor

$\mathbf{V}^{a, P_{b}}$ Velocidad relativa del aire respecto al punto $P_{b}$ de la pala $b$

$\mathbf{V}^{E_{b}} \quad$ Velocidad absoluta del punto $\mathrm{E}_{b}$

$\mathbf{V}^{G B_{b}}$ Vector velocidad del centro de masas de la pala $b$

$\mathbf{v}_{i} \quad$ Velocidad inducida

$V_{H} \quad$ Valor absoluto de la velocidad $\mathbf{V}^{G}$

$v_{T} \quad$ Velocidad del centro de masas del pararrotor en la dirección $y_{T}$ 
$V_{Z} \quad$ Velocidad de la corriente en túnel de viento

$W \quad$ peso del pararrotor

$w \quad$ Velocidad del centro de masas del pararrotor en la dirección $z_{A}$

$w_{T} \quad$ Velocidad del centro de masas del pararrotor en la dirección $z_{T}$

$x \quad$ Posición a lo largo de la cuerda

$\mathbf{x}(\mathrm{t}) \quad$ Vector de estado

$\mathbf{x}_{0} \quad$ Condiciones iniciales del vector de estado

$\mathbf{x}_{i} \quad$ Condición inicial del vector de estado

$\mathbf{x}_{r, e}(t)$ Vector de estado reducido en equilibrio

$x_{c p} \quad$ posición del centro de presiones ( $\%$ de la cuerda)

$x_{G B}$ Componente de la posición del centro de masas de la pala en la dirección $\mathrm{x}_{B}$

$x_{P_{b}} \quad$ coordenada $x_{B b}$ del centro de presión de la pala

$X_{P} \quad$ posición adimensional del centro de presión de la pala

$\mathbf{y}_{e} \quad$ Vector de estado extendido en equilibrio

$y_{i} \quad$ distribución de datos experimentales

$y_{P_{b}} \quad$ coordenada $y_{B b}$ del centro de presión de la pala

\section{Símbolos Griegos}

$\alpha_{b} \quad$ ángulo de ataque de la pala $b$

$\beta_{b} \quad$ Ángulo de deslizamiento del viento respecto a la cuerda de la pala $b$

$\beta \quad$ Ángulo de batimiento de la pala

$\chi \quad$ Error cuadrático medio del ajuste de datos experimental 
$\lambda \quad$ Parámetro de velocidad inducida

$\Phi \quad$ Ángulo de balanceo

$\phi \quad$ Ángulo de incidencia de flujo

$\psi \quad$ ángulo de azimut

$\rho \quad$ densidad del aire

$\sigma_{i} \quad$ error experimental

$\tau \quad$ coeficiente de eficiencia de Glauert

$\tau_{r} \quad$ familia de funciones base para el ajuste de datos experimentales

$\Theta \quad$ Ángulo de cabeceo

$\theta_{b} \quad$ ángulo de paso de la pala $b$

$\theta_{1 C} \quad$ ángulo de paso cíclico longitudinal

$\theta_{1 S} \quad$ ángulo de paso cíclico lateral

$\mu_{z_{A}} \quad$ Parámetro de relación de velocidades

$u x_{i} \quad$ Incertidumbre de la variable $x_{i}$

$\boldsymbol{\omega}_{b} \quad$ velocidad angular de la pala $b$

$\boldsymbol{\omega} \quad$ velocidad angular del pararrotor

$\left.\boldsymbol{\omega}_{b}\right|_{B} \quad$ velocidad angular de la pala $b$ en ejes B

$\omega(\psi)$ Velocidad angular adimensional

$\Omega_{0} \quad$ Valor medio de la velocidad angular experimental

$\omega_{0} \quad$ Velocidad angular de referencia

$\omega_{i} \quad$ Componente $i$ de la velocidad angular del pararrotor. $i=\mathrm{x}, \mathrm{y}, \mathrm{z}$. 
$\Omega_{0 i} \quad$ Condición de velocidad angular inicial

$\omega_{0 i} \quad$ Condición de velocidad angular adimensional inicial

$\zeta \quad$ Ángulo de arrastre de la pala

\section{Subíndices}

A Sistema de referencia A

A1 Sistema de referencia A1

B Palas

B Sistema de referencia B

b Pala

e Equilibrio

H Pararrotor

i Condición inicial

S relativo a la barra del sistema experimental

$\mathrm{T} \quad$ Sistema de referencia tierra

W Sistema de referencia viento

$\mathrm{W}_{1} \quad$ Sistema de referencia intermedio entre el sistema viento y el sistema ligado a las palas

\section{Superíndices}

A Punto de intersección entre el eje de la pala y el eje del pararrotor

a Aerodinámico

$\mathrm{C}_{1} \quad$ Punto de acoplamiento de la celda de carga con la barra del sistema experimental 
$\mathrm{E}_{b} \quad$ Punto de acoplamiento de la pala $b$ al pararrotor

F Punto de aplicación de fuerzas y momentos aerodinámicos sobre el cuerpo del pararrotor

G Centro de masas del pararrotor

i Inercial

O Punto de acoplamiento entre el pararrotor y el sistema de medición

r Reacción

t Acciones transmitidas

$\mathrm{T}_{i} \quad$ Punto de acoplamiento de la barra del sistema de medida

\section{Sistemas de referencia}

$\left[x_{A}, y_{A}, z_{A}\right]$ Sistema de referencia ligado al centro de masas del pararrotor

$\left[x_{A 1 b}, y_{A 1 b}, z_{A 1 b}\right]$ Sistema de referencia rotatorio con la pala $b$ del pararrotor

$\left[x_{B_{b}}, y_{B_{b}}, z_{B_{b}}\right]$ Sistema de referencia solidario a la pala $b$ del pararrotor

$\left[x_{T}, y_{T}, z_{T}\right]$ Sistema de referencia tierra

$\left[x_{W 1_{b}}, y_{W 1_{b}}, z_{W 1_{b}}\right]$ Sistema de referencia intermedio entre el sistema ligado a las pala $b$ y el sistema viento

$\left[x_{W_{b}}, y_{W_{b}}, z_{W_{b}}\right]$ Sistema de referencia viento de la pala $b$ 


\section{Resumen}

El objetivo de la presente tesis es el estudio del comportamiento de un decelerador de alas rotatorias, denominado pararrotor, ante las variaciones cíclicas del ángulo de paso de las palas. En particular, se investiga la posibilidad de controlar el desplazamiento lateral de un pararrotor mediante las variaciones cíclicas del ángulo de paso de las palas, y, extendiendo el análisis, controlar la trayectoria del dispositivo en régimen de descenso, de modo de satisfacer los requerimientos operacionales.

El pararrotor es un decelerador aerodinámico que vuela en régimen de autorrotación, es decir, que no extrae ni comunica energía del medio. La descripción aerodinámica formal de su comportamiento se basa en una adaptación de la teoría de elemento de pala, ya que no se realiza la integral a lo largo de la envergadura si no que se supone la fuerza aerodinámica resultante concentrada en un punto de la pala, en virtud del pequeño alargamiento de las mismas.

El modelo teórico desarrollado comienza con la formulación de las ecuaciones que describen los efectos dinámicos de las variaciones cíclicas del ángulo de paso. Las fuerzas y torques aerodinámicos son estimados mediante un modelo completo de la pala, obteniéndose una expresión matemática que permite conocer la fuerza y el momento a lo largo de una vuelta. Del análisis de las magnitudes modeladas se observa que los efectos dinámicos son sensiblemente menores que los aerodinámicos, por lo que se puede suponer que los últimos son los dominantes en el cálculo de las acciones dinámicas que alteran el comportamiento del pararrotor.

En cuanto al desarrollo experimental, se construyó un modelo a escala con control del ángulo de paso de las palas para ser montado en el túnel de viento, junto a un arreglo adecuado de elementos de medición y adquisición de datos. Con estos ensayos se buscó calcular experimentalmente parámetros característicos que permitieron validar el modelo teórico.

Se demostró que las variaciones cíclicas del ángulo de paso de las palas permiten modificar el torque que se genera sobre el pararrotor y, por lo tanto, es factible utilizar este efecto como elemento de control sobre el dispositivo. 
También se realizaron una serie de simulaciones numéricas, a partir de un modelo promediado de las fuerzas y los momentos sobre el pararrotor a lo largo de una revolución que fue utilizado en el modelo completo. Estas simulaciones permitieron establecer estados de operación cercanos al equilibrio a partir de soluciones obtenidas para un modelo simplificado de la dinámica del pararrotor. 


\section{Introducción}

\subsection{Introducción al concepto de pararrotor}

Los pararrotores son deceleradores aerodinámicos conformados por alas giratorias en régimen de autorrotación. Existen distintos tipos de deceleradores, que se podrían clasificar en aerodinámicos (donde se ubican los pararrotores) y no aerodinámicos (donde se ubican los globos u otros sistemas autopropulsados). La presente tesis hace foco en los primeros, orientándose hacia el control de este tipo de dispositivos para la realización de una variedad de tareas en las que se requiera la utilización de vehículos aéreos no tripulados.

\subsection{Antecedentes bibliográficos}

\subsubsection{Antecedentes bibliográficos en aerodinámica de bajo número de Reynolds y pequeño alargamiento}

Las palas que utiliza un pararrotor son, en general, de pequeño alargamiento. Esto se debe a los requerimientos de almacenamiento que deben disponer estos dispositivos, ya sea para facilitar su traslado o reducir las dimensiones del mismo. Por lo tanto, el régimen aerodinámico en el cual se inscribe contempla esta singularidad: la del pequeño alargamiento de sus alas.

Se destaca este aspecto porque hay factores que hay que incorporar al estudio aerodinámico de las palas, como ser la fuerte componente tridimensional del flujo y el bajo número de Reynolds al que operan las palas.

Entre los primeros trabajos publicados, se destaca el de Bollay [1], quien desarrolló una teoría no lineal para alas rectangulares de pequeño alargamiento. Para ello reemplaza el ala por un sistema de vórtices constantes a lo largo de la envergadura, asumiendo que el downwash es constante. Partiendo de la ley de BiotSavart se estima la velocidad inducida por los vórtices del ala y los de punta de ala obteniendo una expresión para la velocidad inducida por unidad de longitud, y una 
distribución de presiones. Esta teoría demostró tener un buen comportamiento para alargamientos menores a 1, a partir de los cuales comienza a haber discrepancias considerables con los resultados experimentales.

Kuchemann [2] desarrolló una teoría para calcular la sustentación de un ala, cualquiera sea su planta y su alargamiento, modelando la etapa de transición entre alas de pequeño y gran alargamiento. Basa sus fundamentos en la teoría de línea sustentadora de Prandtl y obtiene una expresión para la variación de la posición del centro de presión en función de la cuerda, el alargamiento y el ángulo de torsión de la pala. Analiza los efectos no lineales en la sustentación, mostrando que para pequeño alargamiento la sustentación crece de manera pronunciada con el ángulo de ataque. Incorpora un factor de corrección para el coeficiente de sustentación por efecto de los vórtices de punta de pala.

Carmichael [3] realizó un relevamiento de los trabajos referidos a perfiles de bajo número Reynolds, caracterizando el tipo de flujo según el número de Reynolds, estudiando la generación de la burbuja de separación de la capa límite. Clasifica la información según ensayos en túnel aerodinámico y ensayos en vuelo, para dar lugar a los trabajos de diseño de perfiles de bajo número de Reynolds.

Motorizados por el novedoso interés despertado por el desarrollo de micro vehículos aéreos (MAVs, según las siglas en inglés), Mueller et al. [4] realizaron una caracterización del flujo según el número de Reynolds en perfiles Eppler 61 y Pfenninger 048, logrando identificar distintas configuraciones de estructuras de flujo que se detallan a continuación: para número de Reynolds entre 1.000 y 10.000 observaron una capa límite laminar, con dificultades para convertirse en turbulenta. Para el rango entre 10.000 y 30.000 observaron la formación de capa límite laminar con desprendimientos. Para números de Reynolds 30.000 y 70.000 identificaron la formación de una capa limite laminar con transición a turbulenta. Y para números de Reynolds mayores a 70.000 y menores a 200.000 obtuvieron un flujo laminar con la presencia de una burbuja de separación.

En esta misma línea investigativa, Pelletier y Mueller [5] ensayaron distintas configuraciones alares de pequeño alargamiento a bajo número de Reynolds. Estudiaron el efecto del alargamiento (con una relación entre envergadura y cuerda de 0,5 a 3 ) y el efecto de la curvatura (para placas planas y de $4 \%$ de curvatura). Se destacan como resultados de este documento de referencia el hecho de que a menores alargamientos se tienen ángulos de ataque de pérdida mayores, por lo que se concluye que este tipo de palas tienen un rango de sustentación mayor que las de gran alargamiento. Los autores sostienen que este hecho se debe a que el flujo permanece adherido para mayores ángulos de ataque, y la capa límite laminar deviene en turbulenta, por lo que el desprendimiento de la misma se produce mas cerca al borde de fuga. Desde esta perspectiva, los autores puntualizan que la contribución fundamental para el sostenimiento de este fenómeno la realizan los vórtices de punta 
de pala.

Otro comportamiento en el que se predice la diferencia entre las alas de alargamiento mayor a 4 y aquellas de pequeño alargamiento, es la pendiente del coeficiente de momento del ala, que en el primer caso se muestra negativa y en el segundo positiva.

Torres y Mueller [6] analizaron distintas configuraciones de plantas alares de pequeño alargamiento. Se analizaron las componentes lineales y no lineales de sustentación, generadas por la circulación de flujo y los vórtices de punta de ala respectivamente. Entre las conclusiones de los experimentos se puede citar que el parámetro que tiene principal incidencia en la caracterización de los perfiles es el alargamiento, seguido por la forma de la planta y el número de Reynolds. Se encontró una fuerte dependencia de la posición del centro de presiones con el alargamiento y el ángulo de ataque. Para pequeño alargamiento y pequeño ángulo de ataque, el comportamiento aerodinámico del perfil se asemeja a uno de gran alargamiento, dominando la sustentación el término lineal de la misma. El centro de presiones se encuentra alrededor del $25 \%$ de la cuerda. Para pequeño alargamiento y alto ángulo de ataque los vórtices de punta de ala tienen mayor incidencia respecto a la componente lineal de sustentación y desplazan el centro de presiones hacia el borde de fuga.

\subsubsection{Antecedentes bibliográficos sobre casos naturales}

Los pararrotores son vehículos aéreos inspirados en la biología, que intentan imitar el comportamiento de formas de vuelo que muestra la naturaleza. Es posible incluir entre estos a pájaros, insectos y semillas, incluso algunas de una sola ala como las semillas de algunas frutas. Estas semillas planeadoras, llamadas sámaras, son tal vez los elementos voladores mas simples, estables y eficientes que la naturaleza haya creado. Son concebidas para dispersarse ellas mismas a distancias suficientemente apartadas de su punto de lanzamiento (árbol madre), de manera que los nuevos especímenes no compitan con sus padres en la obtención de sus fuentes de subsistencia (agua, luz, nutrientes, etc.).

Cabe destacar que entre las principales dificultades para adaptar el método de cálculo de helicópteros a la modelización de las sámaras y otros vehículos aéreos se encuentra el bajo número de Reynolds al cuál operan estos aparatos y a la compleja rotación tridimensional a la que están sometidos.

Rosen y Seter [7] desarrollaron un modelo con el objeto de predecir el equilibrio dinámico de un pararrotor tipo Sámara. El modelo se basó en la teoría del Elemento de Pala. Los resultados obtenidos fueron comparados con observaciones publicadas que convalidan el modelo a pesar de las fuentes importantes de incerti- 
dumbre en las mediciones. Posteriormente los mismos autores presentan un modelo numérico para investigar la estabilidad de la autorrotación vertical de la sámara [8], obtenido a partir del método de pequeñas perturbaciones aplicado a las ecuaciones del movimiento de la sámara. Estudiaron la influencia de diferentes parámetros en la estabilidad. Compararon los resultados del modelo con resultados experimentales cualitativos realizados sobre sámaras artificiales diversas.

Crimi [9] realizó un modelo numérico con 11 grados de libertad para describir el movimiento de un dispositivo de una sola ala en estado estacionario. El modelo permitió alcanzar buena efectividad en la caída de la carga útil debido al régimen de descenso constante y al movimiento de rotación periódico. El autor, además mencionó que el cuerpo se mueve con un movimiento donde el ángulo entre el eje vertical del sistema inercial y el eje de rotación propia del cuerpo es constante, y la velocidad de precesión es constante e igual a la velocidad de rotación del cuerpo.

También en el campo numérico, Roccia et. al. [10], desarrolló una herramienta para la simulación numérica de la aerodinámica no lineal e inestacionaria de semillas autorrotantes. Estos casos presentan un mejor desempeño en la mecánica del vuelo, sobre todo en lo que se refiere a la estabilidad y robustez en la iniciación de la deceleración aerodinámica. La utilización del programa elaborado permite obtener resultados numéricos respecto a la sustentación y el momento que genera la pala, y también de cómo se desarrolla el ángulo de ataque a lo largo de la envergadura.

Inspirados en el concepto de la sámara, también se han desarrollado modelos a escala autopropulsados. Se destaca el trabajo de Fregene et. al. [11], donde modeló el comportamiento dinámico y aerodinámico de la sámara y fabricaron un modelo propulsado, denominado samarai. El modelo tiene una envergadura de 0,3 $\mathrm{m}$, pesa $0,2 \mathrm{~kg}$ y gira a $600 \mathrm{RPM}$. Tiene una superficie de control (flap) que varía cíclicamente el ángulo de ataque de la pala, y es utilizado para el control y el guiado. El modelo matemático se realizó a partir de las ecuaciones de la dinámica, y utiliza un vector de estado de 13 elementos (3 posiciones, 3 velocidades, 3 velocidades angulares y 4 cuaterniones). Para la estimación de las variables de estado se filtran las señales adquiridas por los sensores y se utiliza un filtro de Kalman extendido. La figura siguiente presenta una semilla de arce y un modelo de samarai: 


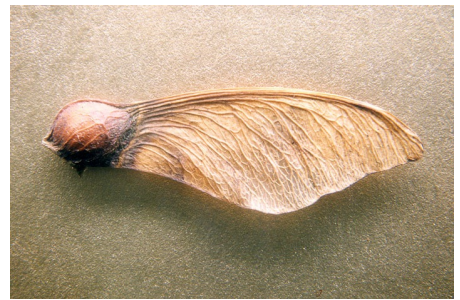

(a) Semilla de arce

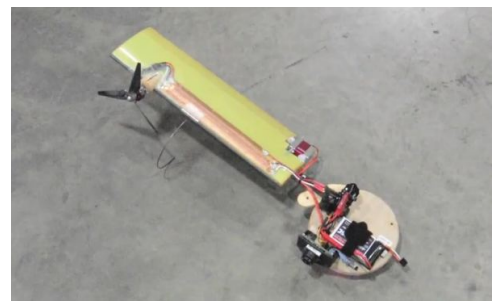

(b) Samarai

Figura 1: Modelo de monocóptero inspirado en la semilla de arce

Kang, en su artículo de 2016 [12], estudió aspectos dinámicos y aerodinámicos de un monocóptero. Utilizó la teoría de elemento de pala para el cálculo de las fuerzas aerodinámicas. Una vez obtenida la expresión diferencial para la sustentación y la resistencia, descompuso las mismas en una dirección paralela y perpendicular al plano de las palas. Estas expresiones se linealizaron y se integraron numéricamente, ya que debido a la complejidad que presentron no era factible su tratamiento analítico. El modelo dinámico se obtuvo a partir de las ecuaciones de la dinámica, incorporando el término aerodinámico desarrollado antes. Para el análisis de la estabilidad consideró que que las perturbaciones son pequeñas y formuló la ecuación de momentos, desacoplando la ecuación que corresponde al eje vertical de las del plano horizontal. De este modo planteó un sistema dinámico no homogéneo, cuya solución depende de sus autovalores. El análisis de estabilidad lo realizó definiendo tres factores: inercial, aerodinámico y de estabilidad marginal. Del análisis se desprende que el momento aerodinámico tiene un efecto estabilizante en el sistema; de otro modo no sería estable.

\subsubsection{Antecedentes bibliográficos sobre casos de aplicación tecnológica}

El desarrollo de la tecnología asociada a los dispositivos autorrotantes tuvo un impulso importante a partir de su introducción en los sistemas de artillería, por un lado, y en aplicaciones espaciales por otro. Numerosos trabajos han sido elaborados para obtener mecanismos de control en deceleradores del tipo paracaídas, que han brindado aportes al modelado y al control de los dispositivos no propulsados.

Algunos trabajos pioneros en el área fueron los desarrollados por Karlsen, Borgström, y Paulsson [13], donde describen la aerodinámica de un cuerpo giratorio provisto de aletas, para ser utilizado como submunición de artillería, y se destacan las ventajas de utilizar alas rígidas en lugar de flexibles.

También Doherr y Schilling [14] han estudiado sistemas de paracaídas giratorios para decelerar municiones. En la referencia mencionada realizan una simulación numérica de la trayectoria y la dinámica del sistema de paracaídas rotato- 
rio. Comentan que los paracaídas rotatorios son muy sensibles a las perturbaciones atmosféricas.

Shpund y Levin han enfocado su estudio a las propiedades aerodinámicas de paracaídas giratorios. Orientaron sus investigaciones a la aplicación de los pararrotores a sistemas de munición que buscan blancos mientras descienden controladamente. El paracaídas se utilizó como mecanismo de control del vuelo. El objetivo fue poder controlar el régimen de descenso, la velocidad de giro y la posición espacial instantánea de la carga. El estudio de la referencia [15] se orientó a entender los efectos de las variables geométricas del paracaídas (relación entre el ancho y el largo de las bandas del paracaídas -relación de aspecto, porosidad geométrica-, y relación entre la longitud de las bandas y la longitud de las cuerdas de suspensión) en la estabilidad dinámica del sistema. Estudiaron paracaídas tipo "cruz". Los resultados presentaron movimientos estables e inestables, dependiendo de las variables mencionadas. Obtuvieron una configuración estable donde el sistema presenta movimiento de precesión, describiendo un movimiento cónico estable y donde la frecuencia dominante es el giro sobre el propio eje. Los autores destacaron la presencia de tres frecuencias dominantes: las dos más bajas relacionadas con las propiedades de inercia y aerodinámicas de la carga y del paracaídas; la tercera relacionada con la velocidad de giro propia, que depende estrechamente de las variables geométricas (las dos primeras varían poco con los cambios en la configuración). Se expusieron también las diferencias que existen entre los ensayos en túnel aerodinámico, donde el movimiento del modelo está restringido, y el vuelo libre. Afirman que, sin embargo, las principales propiedades aerodinámicas, estáticas y dinámicas, y las propiedades de inercia se manifiestan, en ambos casos, de forma cualitativamente igual, aunque no cuantitativamente, y que los resultados de los ensayos en túnel aerodinámico podrían ser empleados para el diseño y análisis de las configuraciones reales. En la referencia [16], los mismos autores investigaron la interferencia aerodinámica entre la carga útil y el paracaídas, para sistemas giratorios y no giratorios. También se estudió el efecto de la geometría del paracaídas. Mencionaron el aumento de la resistencia aerodinámica que se genera en los paracaídas por el movimiento de rotación y la intención de que el paracaídas sirva como medio de control de la trayectoria del sistema paracaídas más carga. Peyada [17] modeló la trayectoria de descenso de paracaídas a partir de la estimación teórica de las derivativas de estabilidad. El modelo que desarrolló cuenta con 9 grados de libertad, y supone al paracaídas y la carga útil como un cuerpo rígido, vinculados por una articulación. Las derivativas, obtenidas a partir de las ecuaciones de la dinámica, fueron utilizadas en la determinación de la trayectoria del sistema bajo distintas configuraciones de frenado. Calise [18] presentó un sistema de control adaptable al amplio rango de condiciones operativas que se requieren de un paracaídas, evaluando distintas estrategias de aproximación al objetivo. El control fue realizado en base a la tasa de cambio del ángulo de nutación, y los resultados obtenidos en los ensayos fueron considerados por los autores como aceptables. 
También en lo referente a la recuperación de sondas en reingreso a la atmósfera, Pepper Jr. [19], presentó un diseño de paracaídas giratorio de altas prestaciones. Se destaca en el trabajo la ventaja del vuelo en autorrotación, en particular, con referencia a la mayor estabilidad giroscópica y resistencia aerodinámica que se obtienen.

Nadal Mora [20], estudió en forma teórica y experimental el descenso en autorrotación axial, y, teóricamente, la dinámica de vuelo de un cilindro provisto de aletas, como el que se muestra en la figura 2. Respecto a la primera línea de investigación, obtuvo una herramienta matemática para predecir el comportamiento de un pararrotor que funciona en un régimen de autorrotación axial, y determinó cuáles son los parámetros que afectan al comportamiento del pararrotor y de qué manera se manifiesta su influencia, entre otros. Respecto al análisis de la dinámica de vuelo, halló un modelo que describe regiones estables e inestables, límites de estabilidad y condiciones de estabilidad para el giro alrededor de un eje próximo a uno de los ejes principales de mayor, menor e intermedio valor de momento de inercia.

Continuando con esta línea, Piechocki [21] desarrolló un modelo teórico de la acción de las fuerzas aerodinámicas sobre el movimiento del cuerpo en rotación que permite identificar los factores de los que dependen los parámetros de funcionamiento del pararrotor y su influencia. También se desarrolló una rutina de simulación numérica a partir del modelado analítico de la dinámica de pararrotores, analizando los efectos del desplazamiento del plano que contiene a las palas respecto al centro de masas.

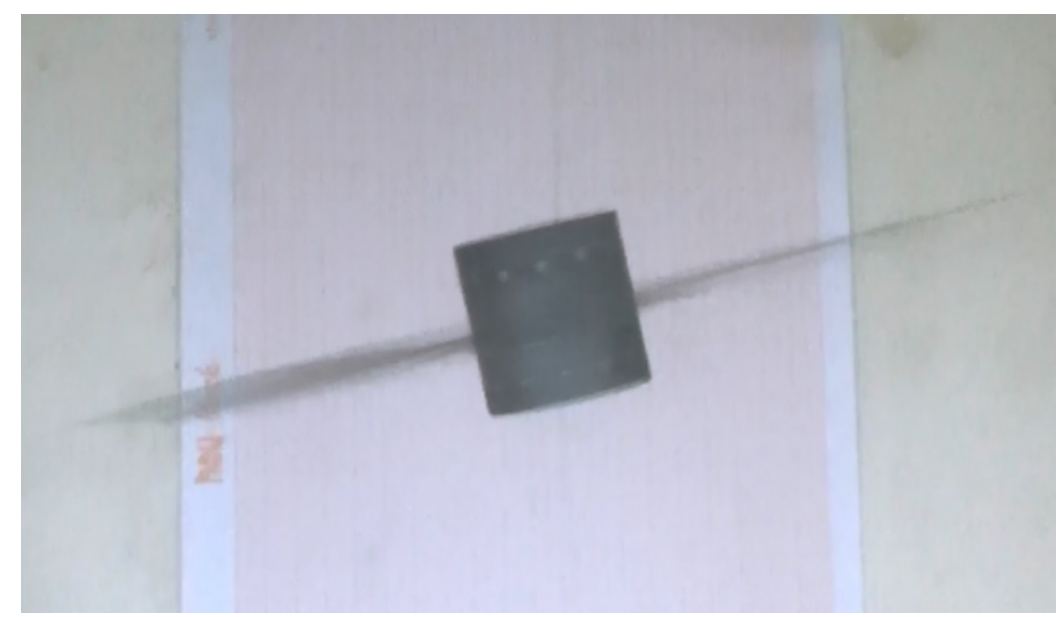

Figura 2: Modelo de pararrotor en túnel vertical

Otro mecanismo que altera el comportamiento de los pararrotores se presenta en [22], en donde se presentan los efectos de modificar la curvatura y el alargamiento de las palas para modificar parámetros operacionales del pararrotor, 
como ser el coeficiente de velocidades y el coeficiente de resistencia del modelo. Se observa experimentalmente que la relación entre la velocidad de descenso y la de rotación se incrementa con la curvatura, mientras que el coeficiente de resistencia del modelo no presenta variaciones significativas. Este punto permite desprender una inmediata aplicación práctica, en la que el pararrotor disminuya la velocidad de giro manteniendo constante la velocidad de descenso. En cambio, se verificó que el incremento en el ángulo de paso de las palas reduce la relación de velocidades.

Taamallah [23] presentó un sistema de control y guiado para helicópteros de pequeña escala en régimen de autorrotación. En comparación con los helicópteros de gran escala, los de tamaño pequeño tienen alta relación potencia/peso, ensamble muy rígido del rotor y alta relación torque/inercia, lo que deriva en regímenes de inestabilidad y de acoplamiento dinámico de mayor relevancia que en los aparatos de gran tamaño. En el planteo del controlador propone un diseño de dos etapas: para el guiado utiliza un modelo de movimiento horizontal, en donde independiza la caída vertical del desplazamiento en el plano perpendicular a la dirección de caída, y reduce el modelo de estados a un vector de 12 componentes (cuerpo rígido). Cada variable del vector de estado, en el caso de movimiento horizontal, es representada por un polinomio, sobre el que se plantea el problema de encontrar el valor de los coeficientes de dicho polinomio de modo de obtener la trayectoria óptima. La optimización se realiza definiendo una función de costo que se busca minimizar. El módulo de control se basa en un método de control robusto: el sistema tiene 4 entradas de control y 6 grados de libertad, por lo que hay 2 grados de libertad sub actuados. Las referencias de seguimiento son posición, velocidad y ángulo de cabeceo (7 en total). La dinámica de las variables posicionales es mucho más lenta que las de velocidad, por lo que plantea un sistema de realimentación del tipo master-slave, donde el ancho de banda del lazo interno es más grande que el externo. Diseña un sistema de control para cada lazo (interno y externo). Para las simulaciones define condiciones iniciales y finales de las variables de referencia, alcanzando condiciones satisfactorias según las condiciones de modelo definidas.

En cuanto a la operación de las palas ante un régimen cíclico de cambio de paso, Li et al. ([24] y [25]) presentaron una serie de trabajos experimentales en base a ensayos sobre una turbina eólica. El ángulo de paso de la turbina es controlado por un plato oscilante, que permite definir tanto el ángulo de paso cíclico como colectivo. Para la medición de las fuerzas y los momentos sobre la turbina se utiliza una celda de carga de 6 ejes. El trabajo se dividió en tres partes: la primera es la caracterización de los parámetros de desempeño de la turbina, para investigar la relación entre los parámetros de desempeño (coeficiente de potencia y de empuje) y el cociente entre la velocidad de rotación y la velocidad de la corriente, para distintos ángulos de paso. La segunda parte es la caracterización de los parámetros según la variación colectiva del ángulo de paso, y por último la caracterización de dichos coeficientes según variaciones cíclicas del ángulo de paso de las palas. 
Respecto al primer eje del trabajo, concluyeron que existe un valor óptimo para el cociente entre la velocidad de rotación y la velocidad de la corriente, asociado a la variación del ángulo de ataque de la pala. En cuanto al desempeño de los coeficientes de potencia y empuje respecto al ángulo de paso colectivo, se observó que existe un ángulo de paso óptimo en el que estos coeficientes se maximizan. Los autores indican que esta optimización tiene relación con la fluctuación del ángulo de ataque y el desprendimiento del flujo alrededor del perfil. Respecto a la tercera parte del trabajo, los coeficientes de empuje y de potencia no presentaron variaciones significativas a medida que se modifica el ángulo de paso cíclico. Sí se observaron modificaciones sustanciales en los coeficientes de momentos de cabeceo y guiñada, por lo que se demuestra posible controlar el momento sobre la turbina mediante acciones cíclicas en el ángulo de paso. La modificación del ángulo de incidencia del flujo respecto al eje de la turbina altera principalmente el ángulo de ataque de las palas. Los coeficientes de empuje y de potencia no se ven alterados por el cambio en el ángulo de incidencia del flujo, y la utilización del ángulo de paso cíclico permite compensar el efecto del viento cruzado en la turbina.

El desarrollo de la tecnología aeroespacial y la necesidad de reutilizar los vehículos espaciales (lanzadores, satélites, módulos) impulsaron la creación de distintas alternativas de recuperación de dichos vehículos. Por el lado de los sistemas de alas rotatorias, se destacan trabajos de la NASA de las décadas de 1960 y 1970, donde se investigó un concepto de vehículo de entrada planetaria basado en el concepto de pararrotor, que daba a los astronautas la habilidad de maniobrar y aterrizar el vehículo de manera precisa y segura; y trabajos privados [26] que dotaron de palas a un cohete para su recuperación. Las siguientes figuras muestran algunos de los modelos diseñados:

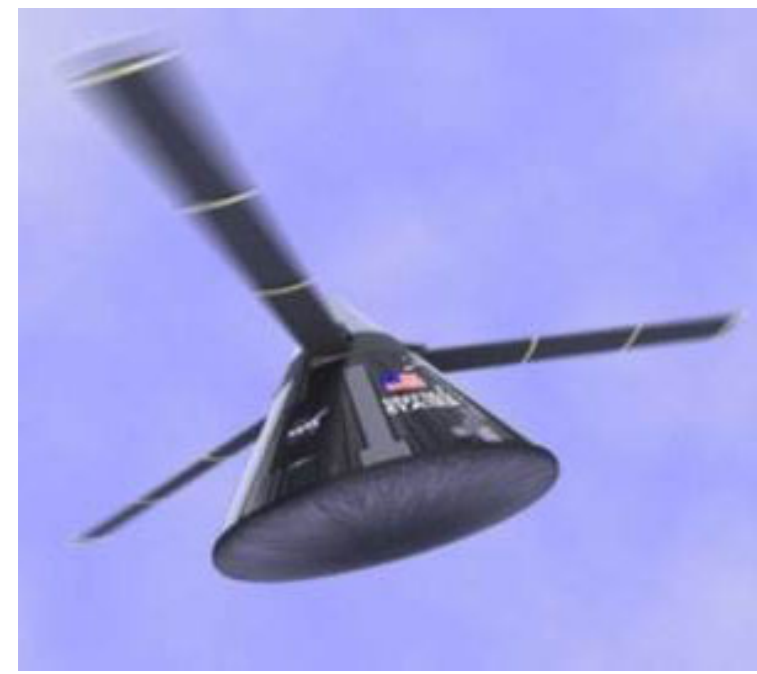

(a) Modelo NASA

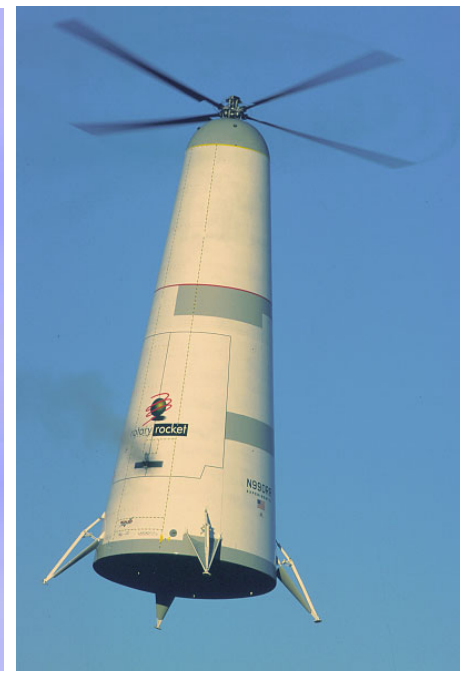

(b) Rotary Rocket

Figura 3: Variantes autorrotantes para recuperación de sondas 


\subsection{Aplicaciones}

El desarrollo de las investigaciones sobre pararrotores se sustenta en la necesidad de frenar de manera controlada el descenso de un cuerpo bajo ciertas condiciones atomosféricas. Entre las aplicaciones para las que es necesario frenar la caída de manera controlada podemos listar las siguientes:

- recuperación de elementos provenientes del espacio exterior,

- control de trayectorias de proyectiles,

- control de sondas meteorológicas,

- ingreso de vehículos en atmósferas extraterrestres.

Las tareas de caracterización atmosférica (relevamiento de presión, humedad, viento, contaminantes, etc) presentan un creciente interés medioambiental, que va de la mano del desarrollo de actividades productivas y económicas sustentables. Si bien existen diversos métodos como la utilización de globos, sistemas electromagnéticos o sistemas embarcados, la utilización de pararrotores para estos fines es una alternativa viable debido a las ventajas prácticas y económicas que presentan.

En lo referido al sistema de ingreso de dispositivos en atmósferas, se debe tener en cuenta la robustez, simplicidad y economía en la utilización.

También la industria armamentista presenta constantes avances en este tipo de tecnologías, ya que estos dispositivos permiten el control remoto, con lo que las operaciones son no tripuladas. Empresas como Lockheed Martin y Textron presentan desarrollos al respecto en los que se destacan el bajo peso, la facilidad en el ensamble y la sencillez de los mecanismos. 


\section{Estudio teórico del desplazamiento lateral de un decelerador aerodinámico a través de las variaciones cíclicas del paso de las palas}

\subsection{Introducción}

El presente capítulo tiene como objetivo estudiar teóricamente el comportamiento dinámico de un pararrotor en vuelo libre ante las variaciones cíclicas del ángulo de paso de las palas. Para esto, se describe la geometría de un pararrotor, junto a las magnitudes cinemáticas necesarias para la determinación de su posición y velocidad.

A partir del planteo de las ecuaciones de Newton-Euler sobre el cuerpo cilíndrico del pararrotor, se busca llegar a la expresión de las mismas en su forma canónica. Para esto, se desglosa el análisis, considerando las fuerzas que actúan sobre el cuerpo cilíndrico, las fuerzas que actúan sobre las palas, las condiciones de ligadura cinemática entre las variables de estado y la ecuación de trayectoria del centro de masas del pararrotor.

Una vez obtenida la ecuación de estado del sistema, se analizan y asignan modelos a cada uno de los elementos que la constituyen. Los efectos inerciales y aerodinámicos son estudiados a efectos de asignarles expresiones matemáticas que permitan su inclusión para el cálculo. Distintos modelos simplificativos son estudiados, además de la evaluación del desarrollo en series de Taylor de las expresiones obtenidas.

Con las expresiones obtenidas se resuelve la ecuación de estado del pararrotor en vuelo libre, y se particulariza su solución para el caso de ensayo de un pararrotor en túnel de viento. 


\subsection{Autorrotación}

A modo introductorio se describe el fenómeno de autorrotación, que es el principio sobre el cual se basa el desempeño como desacelerador del pararrotor.

La autorrotación es la rotación continua de un cuerpo inmerso en un flujo sin otras fuentes externas de energía más que la propia acción de la corriente de fluido. Bajo esta definición, los molinos de viento, turbinas hidráulicas, anemómetros de cazoletas, frutos de especies vegetales tipo sámaras, algunos tipos de extractores de aire, funcionan bajo el principio de autorrotación. Todos estos dispositivos giran puramente por efecto de fuerzas fluidodinámicas, sin fuentes externas de energía; están conformados geométricamente de tal manera que, cuando se sitúan en el seno de una corriente de fluido, se crea un par que inicia la rotación cuando se libera el cuerpo permitiéndole girar libremente. En algunos casos, se requiere un impulso que inicie el movimiento. En los ejemplos mencionados también se observa que el eje de rotación del cuerpo puede ser paralelo o perpendicular a la dirección de la corriente, generando distinto tipo de movimiento (periódico o constante). También puede ocurrir que un cuerpo se mueva libremente en un fluido, sin que su eje de rotación tenga impedido ningún movimiento e incluyendo efectos giroscópicos.

El principio de la autorrotación es ampliamente conocido por su aplicación al caso de helicópteros. Representa un caso especial de operación en el descenso del rotor, en el que los helicópteros descienden con velocidad constante. En este caso, realizando un balance de energía se puede concluir que la potencia que el rotor necesita para generar la tracción que equilibra al peso se obtiene de la disminución de la energía potencial del helicóptero. Desde el punto de vista de la operación la autorrotación se traduce en una maniobra que en caso de falla del motor del vehículo, permite descenderlo con velocidad controlada. La maniobra de aterrizaje en régimen de autorrotación se divide en tres fases: una fase inicial no estacionaria en la que la disminución del ángulo de paso colectivo incrementa la velocidad de rotación del rotor y la energía cinética del mismo; una segunda fase estacionaria en la que el descenso se produce a velocidad constante; y una tercera y última fase no estacionaria en la que, mediante el incremento del ángulo de paso colectivo se produce un par sobre el rotor que conlleva la deceleración angular que produce la desaceleración brusca en el descenso del vehículo.

En el caso de los pararrotores, la diferencia fundamental se encuentra en el alargamiento de las palas. Sin embargo, el establecimiento del régimen de autorrotación se da de la misma manera: la corriente de aire acelera el rotor hasta alcanzar el régimen estacionario, momento en el que las fuerzas aerodinámicas tienen resultante en la dirección del flujo incidente.

Una primera aproximación para la descripción de este fenómeno es la que puede obtenerse en base a la teoría de cantidad de movimiento [27]. Si se modelan 
las palas como un disco resistente que se desplaza con velocidad de descenso $V_{Z}$, en el caso de la autorrotación la tracción es igual a la resistencia aerodinámica. A partir de plantear esta igualdad, es posible obtener la siguiente expresión para la velocidad de descenso en régimen de autorrotación ideal:

$$
V_{z}=-1,77 \sqrt{\frac{W}{2 \rho S}}
$$

Donde $W$ es el peso del helicóptero, $\rho$ la densidad del aire y $S$ la superficie barrida por las palas. La evaluación de esta expresión para los valores promedio de los parámetros que se utilizan en los pararrotores estudiados tiene como resultado valores de velocidad de descenso del orden de los $-5 \mathrm{~m} / \mathrm{s}$, que son consistentes con los obtenidos mediante modelos desarrollados a partir de teorías más complejas, como las que se desarrollan en el anexo de modelos aerodinámicos y que incluyen parámetros de control, aerodinámicos u operacionales.

\subsection{Definiciones generales del modelo teórico}

El pararrotor es un sistema multicuerpo, constituido por diferentes elementos que, en principio, presentan movimientos relativos entre si. Por este motivo es conveniente definir adecuadamente cada uno de los elementos que lo componen, como así también sistemas de referencia solidarios a cada uno de ellos, para poder expresar las fuerzas y momentos, como también para desarrollar las ecuaciones de Newton-Euler según sea conveniente.

A los fines de modelar geométricamente el pararrotor, se denomina $H$ al cuerpo cilíndrico del pararrotor, y $b=1,2$ a cada una de las palas del mismo (ver figura 4). Cada una de las palas se mueven solidarias al cilindro acopladas en el punto $E_{b}$, alrededor del cual pueden girar un ángulo $\theta_{b}$ (ángulo de paso de cada pala) en la dirección del eje de la pala. El punto de acoplamiento $E_{b}$ se encuentra a una distancia denominada $e$ respecto al eje del cilindro.

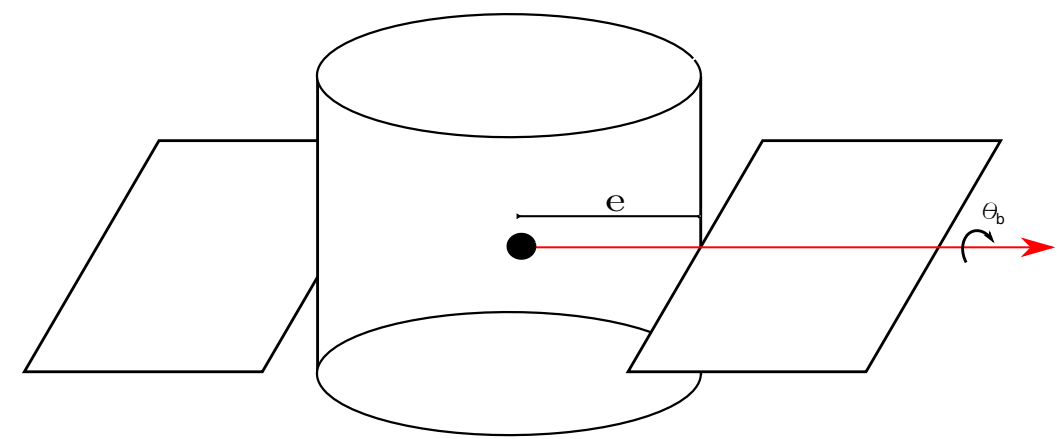

Figura 4: Esquema del pararrotor y definición del ángulo de paso. 
Se define un sistema de referencia inercial fijo compuesto por la terna $\left[x_{T}, y_{T}, z_{T}\right]$, denominado sistema tierra e indicado con la letra $\mathrm{T}$, y un sistema ligado al cuerpo del pararrotor denominado A1b, cuyo eje $x_{A 1 b}$ coincide con el eje de una de las palas. El eje $z_{A 1 b}$ está alineado con el eje de simetría axial del cuerpo cilíndrico, y el eje $y_{A 1 b}$ forma un triedro derecho con los ejes $x_{A 1 b}$ y $z_{A 1 b}$. Esta terna móvil se indica como $\left[x_{A 1 b}, y_{A 1 b}, z_{A 1 b}\right]$. El ángulo de paso de las palas $\theta_{b}$, determina un sistema solidario a la pala $b$ indicado como $\left[x_{B b}, y_{B b}, z_{B b}\right]$. El sistema $B_{b}$ resulta de girar un ángulo $\theta_{b}$ el sistema A1 según el eje $x_{A 1 b}$.

La transformación de un sistema a otro se realiza mediante matrices que permiten transformar magnitudes de un sistema de ejes a otro sistema, además de posicionar el cuerpo del pararrotor en el espacio. La posición del dispositivo queda definida mediante el vector de posición del centro de masas del cuerpo del pararrotor $\mathbf{r}^{G}(t)$, mientras que la velocidad del centro de masas del cuerpo del pararrotor es $\mathbf{V}^{G}(t)$ y la velocidad angular del cuerpo del pararrotor es $\boldsymbol{\omega}^{G}(t)$.

Según sus componentes, la posición, velocidad y velocidad angular resultan:

$$
\begin{gathered}
\mathbf{r}^{G}(t)=r_{G_{1}}(t) \mathbf{i}_{T}+r_{G_{2}}(t) \mathbf{j}_{T}+r_{G_{3}}(t) \mathbf{k}_{T} \\
\mathbf{V}^{G}(t)=u(t) \mathbf{i}_{A}+v(t) \mathbf{j}_{A}+w(t) \mathbf{k}_{A} \\
\boldsymbol{\omega}^{G}(t)=\omega_{x}(t) \mathbf{i}_{A}+\omega_{y}(t) \mathbf{j}_{A}+\omega_{z}(t) \mathbf{k}_{A}
\end{gathered}
$$

Donde $\mathbf{r}^{G}, \mathbf{V}^{G}$ y $\boldsymbol{\omega}^{G}$ representan la posición del centro de masas, la velocidad del centro de masas y la velocidad angular del cuerpo del pararrotor. La transformación del sistema $\mathrm{T}$ al A1b se define mediante rotaciones sucesivas, que se describen a continuación. En primer lugar, se define un sistema de referencia en ejes cuerpo, denominado sistema A, que es producto de la rotación de los ángulos de Euler: un primer giro, indicado mediante $\Phi$ y un segundo giro, indicado mediante $\Theta$. Por último, un tercer giro indicado mediante $\psi$, determina el sistema de referencia A1b. Definidos de esta manera, la primera rotación es respecto al eje $y_{T}$, la segunda respecto al eje $x_{T 1}$ y la tercera respecto al $z_{A}$. En la bibliografía de referencia [28] esta rotación se reconoce como 2-1-3. 

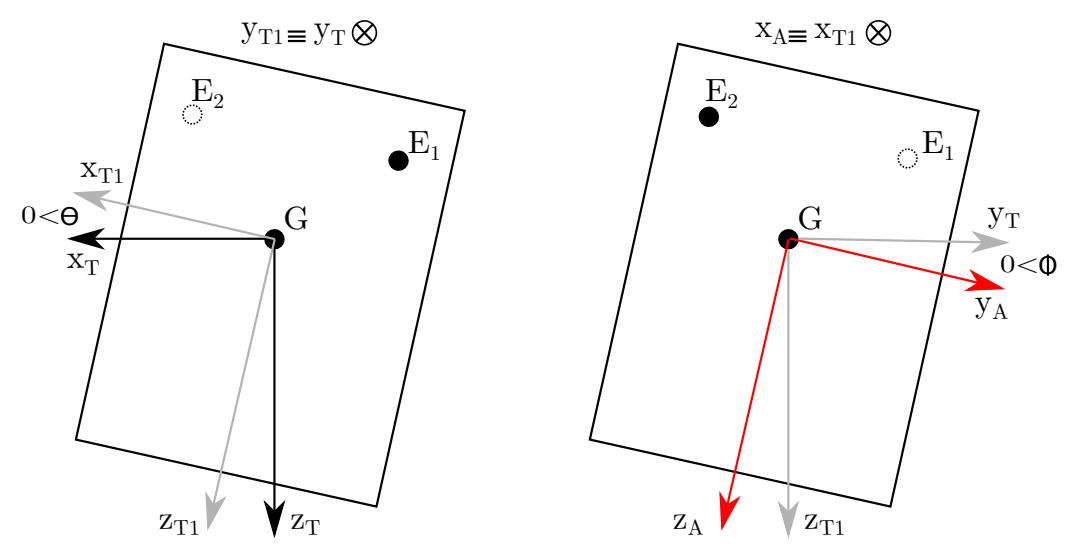

Figura 5: Definición de los sistemas T1 y A para una posición azimutal $\psi$ arbitraria

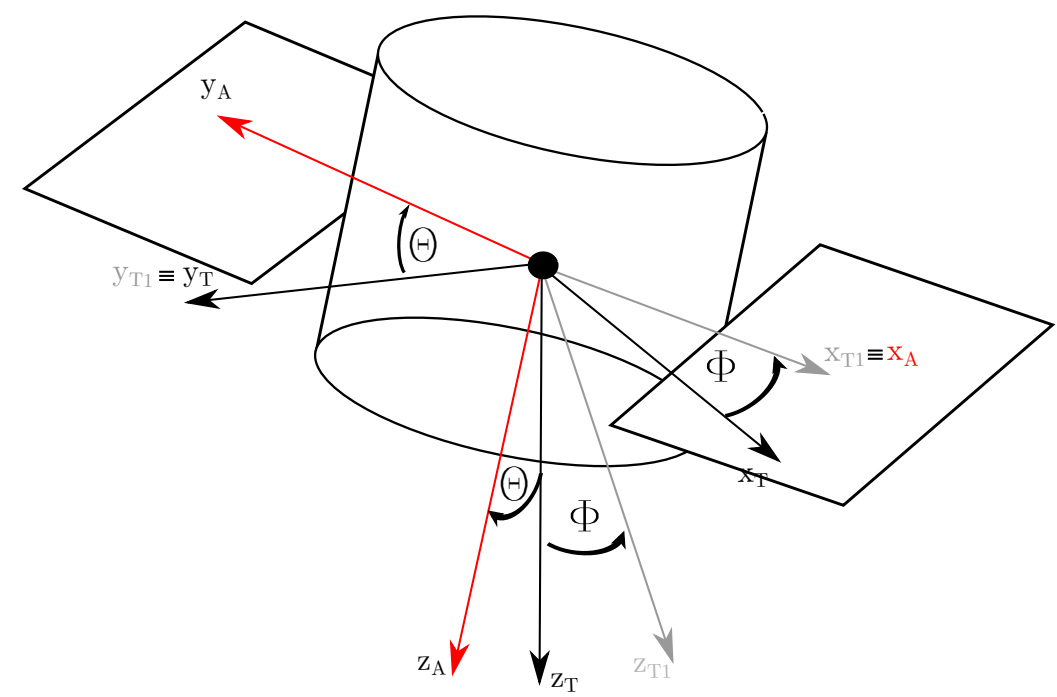

Figura 6: Definición tridimensional de los sistemas T1 y A para una posición azimutal $\psi$ arbitraria .

La transformación del sistema en ejes tierra al sistema A1b viene dada por el producto $\mathbf{T}=\left[\mathbf{T}_{A 1 b A}\right]\left[\mathbf{T}_{A T 1}\right]\left[\mathbf{T}_{T 1 T}\right]$, siendo las matrices de transformación para cada giro las siguientes:

$$
\left[\mathbf{T}_{T 1 T}\right]=\left[\begin{array}{ccc}
\cos \Theta(t) & 0 & -\sin \Theta(t) \\
0 & 1 & 0 \\
\sin \Theta(t) & 0 & \cos \Theta(t)
\end{array}\right],\left[\mathbf{T}_{A T 1}\right]=\left[\begin{array}{ccc}
1 & 0 & 0 \\
0 & \cos \Phi(t) & \sin \Phi(t) \\
0 & -\sin \Phi(t) & \cos \Phi(t)
\end{array}\right]
$$




$$
\begin{gathered}
{\left[\mathbf{T}_{A 11 A}\right]=\left[\begin{array}{ccc}
\cos \psi(t) & \sin \psi(t) & 0 \\
-\sin \psi(t) & \cos \psi(t) & 0 \\
0 & 0 & 1
\end{array}\right]} \\
{\left[\mathbf{T}_{A 12 A}\right]=\left[\begin{array}{ccc}
\cos [\psi(t)+\pi] & \sin [\psi(t)+\pi] & 0 \\
-\sin [\psi(t)+\pi] & \cos [\psi(t)+\pi] & 0 \\
0 & 0 & 1
\end{array}\right]=\left[\begin{array}{ccc}
-\cos \psi(t) & -\sin \psi(t) & 0 \\
\sin \psi(t) & -\cos \psi(t) & 0 \\
0 & 0 & 1
\end{array}\right]}
\end{gathered}
$$

Se definen sobre el pararrotor una serie de puntos de interés, a los efectos de disponer de referencias para expresar las fuerzas, momentos y demás magnitudes. Las principales referencias geométricas del modelo se muestran en la siguiente imagen:

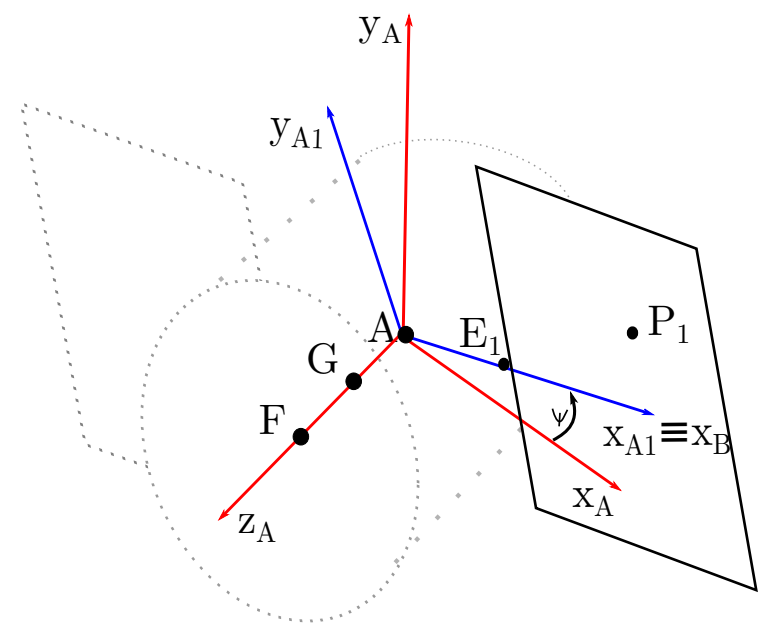

Figura 7: Puntos de referencia geométrica del modelo para la expresión de magnitudes dinámicas y cinemáticas.

donde

- $\mathrm{P}_{1}$, es el centro de presiones de la pala 1 ,

- $\mathrm{E}_{1}$, es el punto de acoplamiento de la pala 1 , 
- A, es el punto de intersección entre el eje de las palas y el eje del cilindro,

- G, es el centro de masas del cuerpo del pararrotor,

- F, es el punto de aplicación de fuerzas y momentos aerodinámicos del cilindro del pararrotor.

Con esta convención podemos definir a $\mathbf{E}_{1} \mathbf{P}_{1}=x_{P_{1}} \mathbf{i}_{B_{1}}+y_{P_{1}} \mathbf{j}_{B_{1}}+z_{P_{1}} \mathbf{k}_{B_{1}}$ como el vector posición del centro de presiones de la pala 1 respecto al punto de acoplamiento en el sistema $B_{1}$, y a $\mathbf{A E}_{1}=e \mathbf{i}_{A 11}$ el vector posición del punto de acoplamiento de la pala 1 respecto al punto $A$ en el sistema $A 11$. De la misma manera se pueden definir las referencias de la pala 2 . También se observa que el punto al cual se transmiten las acciones de las palas (punto $A$ ) se encuentra desplazado mediante el vector $\mathbf{G A}=-L \mathbf{k}_{A}$.

De manera complementaria se define la matriz de transformación del sistema de ejes pala $\left[B_{b}\right]$ al sistema de ejes $[A 1 b]$ :

$$
\left[T_{A 1 b B_{b}}\right]=\left[\begin{array}{ccc}
1 & 0 & 0 \\
0 & \cos \theta_{b} & \sin \theta_{b} \\
0 & -\sin \theta_{b} & \cos \theta_{b}
\end{array}\right]
$$

Matriz de transformación del sistema $B_{b}$ al sistema A1b

En cuanto a la nomenclatura utilizada para las fuerzas y los momentos, se utilizará a partir de aquí un esquema que contenga toda la información necesaria para su correcta interpretación en un único símbolo. En el caso de tratarse de un vector, se indicará resaltando la variable mediante el uso de negrita. Los superíndices utilizados indican la naturaleza de la acción (aerodinámica, inercial, gravitatoria, transmitida) y, si fuera necesario, el punto de aplicación de dicha acción. Los subíndices indican sobre que agente se aplica dicha acción (pala, cuerpo del pararrotor, etc.) y el sistema de referencia o la dirección en la cual está expresada. Por ejemplo, la magnitud $\mathbf{M}_{b, A}^{a, G}$ indica el momento aerodinámico respecto al punto $\mathrm{G}$ que genera la pala $b$, expresado en el sistema de referencia A.

\subsection{Modelo dinámico del pararrotor en vuelo libre}

Para la obtención de las ecuaciones que describen el movimiento del pararrotor en vuelo libre se consideran los distintos elementos que lo constituyen y 
las fuerzas que se transmiten entre ellos, ya sean de origen aerodinámico, inercial o gravitatorio. En primer lugar, se analizan las fuerzas y momentos sobre el cuerpo cilíndrico, denominado $H$, incorporando los efectos aerodinámicos, inerciales y gravitatorios sobre este cuerpo. Una vez concluido este estudio, se analizan las fuerzas y momentos que se generan en las palas, discriminándolas en aquellas que tienen origen aerodinámico, inercial o gravitatorio. La manera en la que estos fenómenos modifican el comportamiento del pararrotor también es analizada en el apartado correspondiente.

Se utilizarán de aquí en adelante, las siguientes variables de estado para caracterizar al sistema:

- $u, v$ y $w$, son las componentes del vector velocidad absoluta del centro de masas del cuerpo del pararrotor expresada en el sistema de referencia A.

- $\omega_{x}, \omega_{y} \mathrm{y} \omega_{z}$, son las velocidades angulares del cuerpo del pararrotor en el sistema A.

- $\Theta, \Phi$ y $\psi$, son los ángulos de Euler entre el sistema tierra y el sistema A.

- $r_{G_{1}}, r_{G_{2}}$ y $r_{G_{3}}$, son las coordenadas de la posición del centro de masas del pararrotor en el sistema tierra.

Se define el vector de control como $\mathbf{u}_{C}(t)$, cuyas componentes son $\theta_{0}$ (ángulo de paso colectivo), $\theta_{1 S}$ y $\theta_{1 C}$ (ángulos de paso cíclico longitudinal y lateral, respectivamente).

Las secciones subsiguientes detallan la relación entre las variables de estado y de control definidas a partir de las ecuaciones de Newton-Euler, la relación de ligadura cinemática entre ellas y las ecuaciones de trayectoria del centro de masas del pararrotor.

\subsection{Fuerzas sobre el cilindro}

Para analizar las fuerzas y momentos aplicadas sobre el cilindro se explicitan las fuerzas y momentos que las palas transmiten al cuerpo cilíndrico. A tal fin, se utiliza la figura 8, donde se manifiestan las acciones (fuerzas y momentos) que realizan las palas sobre el cuerpo del pararrotor.

Sobre los puntos $E_{1}$ y $E_{2}$ se aplican las fuerzas que generan las palas (identificadas como $\mathbf{F}_{1}^{t}$ y $\mathbf{F}_{2}^{t}$ ) y los momentos que las mencionadas fuerzas generan sobre estos puntos (identificados como $\mathbf{M}_{1}^{t, E_{1}}$ y $\mathbf{M}_{1}^{t, E_{2}}$ ). Estas acciones están aplicadas sobre el punto $E_{b}$, que, en principio, no coincide con el punto A. 


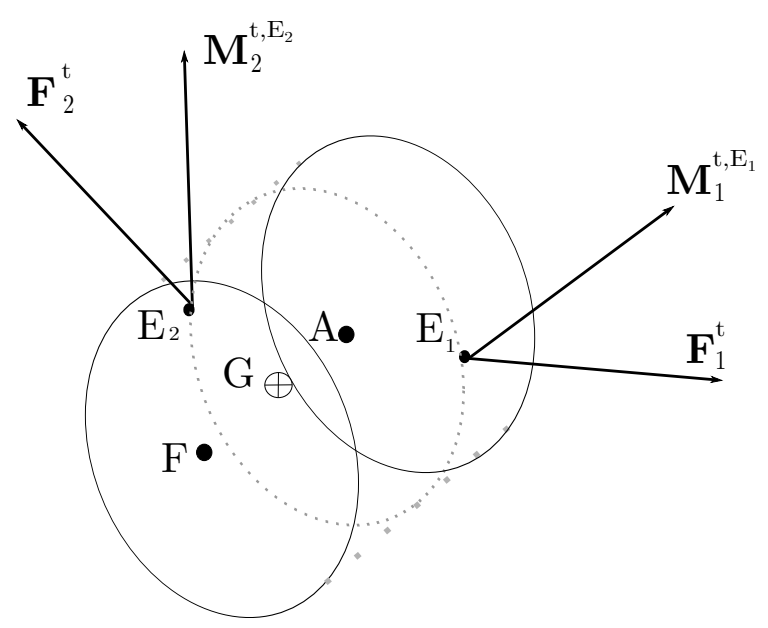

Figura 8: Fuerzas de la pala transmitidas al cilindro del pararrotor

Estas componentes pueden expresarse como:

$$
\begin{gathered}
\mathbf{F}^{t}=\mathbf{F}_{1}^{t}+\mathbf{F}_{2}^{t} \\
\mathbf{M}^{t, A}=\mathbf{M}_{1}^{t, E_{1}}+\mathbf{M}_{2}^{t, E_{2}}+\mathbf{A E}_{1} \wedge \mathbf{F}_{1}^{t}+\mathbf{A E}_{2} \wedge \mathbf{F}_{2}^{t}=\mathbf{M}^{a, A}+\mathbf{M}^{g, A}+\mathbf{M}^{i, A}
\end{gathered}
$$

donde los superíndices $t$ indican acciones transmitidas al cuerpo del pararrotor.

Si consideramos el cilindro como un cuerpo rígido con masa $M_{H}$, se pueden introducir sobre el cuerpo, además de las fuerzas que transmiten las palas, las fuerzas y momentos aerodinámicos y gravitatorios propios del cilindro, tal como se observa en la siguiente figura:

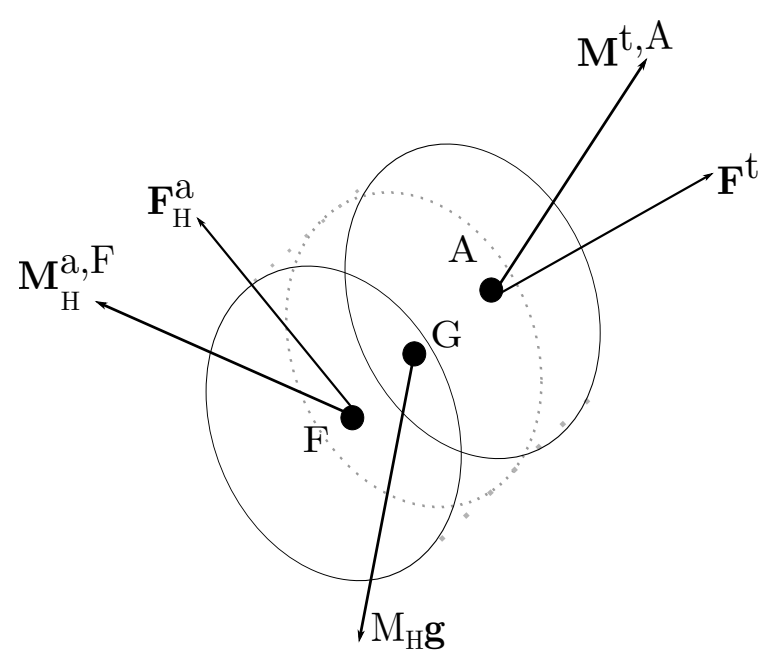

Figura 9: Diagrama de fuerzas y momentos sobre el cilindro 
En la figura 9 se identifican las fuerzas y momentos que se aplican al cilindro del pararrotor. La fuerza gravitatoria, indicada como $M_{H} \mathbf{g}$ en la figura 9, está aplicada en el centro de masas $\mathrm{G}$ del cilindro, y las fuerzas y momentos aerodinámicos que provoca el cuerpo cilindrico se indican mediante los vectores $\mathbf{F}_{H}^{a}$ y $\mathbf{M}_{H}^{a, F}$ aplicados en el punto $\mathrm{F}$. Con la ayuda de este diagrama de fuerzas es posible formular las ecuaciones de Newton-Euler correspondientes a la dinámica en vuelo libre referidas al centro de masas del cuerpo del pararrotor como sigue:

$$
\begin{gathered}
\mathbf{F}_{H}^{a}+\mathbf{F}^{t}+M_{H} \mathbf{g}=M_{H} \frac{d \mathbf{V}^{G}}{d t} \\
\mathbf{M}_{H}^{a, A}+\mathbf{M}^{t, A}+\mathbf{G A} \wedge \mathbf{F}^{t}+\mathbf{G F} \wedge \mathbf{F}_{H}^{a}=\frac{d \mathbf{h}_{H}}{d t}
\end{gathered}
$$

El miembro derecho de la ecuación (6) representa la variación temporal del momento cinético del cilindro del pararrotor. Esta magnitud se calcula como:

$$
\left.\mathbf{h}_{H}\right|_{A 11}=\left.\mathbf{I}_{H} \boldsymbol{\omega}\right|_{A 11}
$$

siendo $\left.\boldsymbol{\omega}\right|_{A 11}$ la velocidad angular del pararrotor expresada en el sistema de referencia del cuerpo e $\mathbf{I}_{H}$ el tensor de inercia del cuerpo, que es diagonal si los ejes de inercia son principales. La validez de la ecuación (7) se circunscribe al caso en el que, tanto el tensor $\mathbf{I}_{H}$ y $\boldsymbol{\omega}$ se encuentran definidos en el sistema A11.

Redefiniendo :

$$
\begin{gathered}
\mathbf{F}_{H}^{g}=M_{H} \mathbf{g} \\
\mathbf{F}_{H}^{i}=-M_{H} \frac{d \mathbf{V}^{G}}{d t} \\
\mathbf{M}^{t, G}=\mathbf{M}^{t, A}+\mathbf{G} \mathbf{A} \wedge \mathbf{F}^{t} \\
\mathbf{M}_{H}^{a, G}=\mathbf{M}_{H}^{a, F}+\mathbf{G F} \wedge \mathbf{F}_{H}^{a} \\
\mathbf{M}_{H}^{i, G}=-\frac{d \mathbf{h}_{H}}{d t}
\end{gathered}
$$

se pueden reescribir las ecuaciones (5) y (6) como sigue:

$$
\begin{aligned}
& \mathbf{F}^{t}+\mathbf{F}_{H}^{g}+\mathbf{F}_{H}^{a}+\mathbf{F}_{H}^{i}=0 \\
& \mathbf{M}^{t, G}+\mathbf{M}_{H}^{a, G}+\mathbf{M}_{H}^{i, G}=0
\end{aligned}
$$


Las expresiones (8) y (9) describen la dinámica de vuelo libre de un pararrotor. En ellas se identifica el aporte que hace cada uno de los elementos constitutivos del pararrotor a la dinámica de vuelo.

\subsection{Ligadura cinemática}

Las grupos de variables de estado definidas como velocidades angulares $\left(\omega_{x}, \omega_{y}\right.$ y $\left.\omega_{z}\right)$ y ángulos de Euler $(\Theta, \Phi$ y $\psi)$ no son independientes entre si. Considerando la definición del los ángulos de Euler en la figura 5 se puede relacionar la velocidad angular $\boldsymbol{\omega}(t)$ con la tasa de cambio temporal de los ángulos de Euler como:

$$
\begin{gathered}
\boldsymbol{\omega}=\frac{d \Theta}{d t} \mathbf{j}_{T 1}+\frac{d \Phi}{d t} \mathbf{i}_{A}+\frac{d \psi}{d t} \mathbf{k}_{A} \\
{\left[\begin{array}{c}
\omega_{x} \\
\omega_{y} \\
\omega_{z}
\end{array}\right]=\left[\mathbf{T}_{A T 1}\right]\left[\begin{array}{c}
0 \\
\frac{d \Theta}{d t} \\
0
\end{array}\right]+\left[\begin{array}{c}
\frac{d \Phi}{d t} \\
0 \\
0
\end{array}\right]+\left[\begin{array}{c}
0 \\
0 \\
\frac{d \psi}{d t}
\end{array}\right]}
\end{gathered}
$$

resultando:

$$
\left[\begin{array}{l}
\omega_{x} \\
\omega_{y} \\
\omega_{z}
\end{array}\right]=\left[\begin{array}{ccc}
0 & 1 & 0 \\
\cos \Phi & 0 & 0 \\
-\sin \Phi & 0 & 1
\end{array}\right]\left[\begin{array}{l}
\frac{d \Theta}{d t} \\
\frac{d \Phi}{d t} \\
\frac{d \psi}{d t}
\end{array}\right]
$$

En la ecuación (12) se identifica la matriz [LC] como:

$$
\mathbf{L C}=\left[\begin{array}{ccc}
0 & 1 & 0 \\
\cos \Phi & 0 & 0 \\
-\sin \Phi & 0 & 1
\end{array}\right]
$$

que se invierte como:

$$
\left[\mathbf{L C}^{-1}\right]=\left[\begin{array}{ccc}
0 & \cos \Phi^{-1} & 0 \\
1 & 0 & 0 \\
0 & -\frac{\sin \Phi}{\cos \Phi} & 1
\end{array}\right]
$$


y dando lugar a:

$$
\begin{gathered}
\frac{d \Theta}{d t}=\frac{1}{\cos \Phi} \omega_{y} \\
\frac{d \Phi}{d t}=\omega_{x} \\
\frac{d \psi}{d t}=-\frac{\sin \Phi}{\cos \Phi} \omega_{y}+\omega_{z}
\end{gathered}
$$

De la ecuación (15c) se desprende que la tasa de cambio temporal del ángulo $\psi$ no es exactamente $\omega_{z}(t)$, si no que también está compuesta por una fracción de $\omega_{y}(t)$.

\subsection{Ecuaciones de la trayectoria del centro de masas del cuerpo del pararrotor}

La trayectoria del centro de masas del pararrotor puede describirse mediante el sistema de ecuaciones diferenciales:

$$
\frac{d \mathbf{r}^{G}(t)}{d t}=\mathbf{V}^{G}(t)
$$

La integración temporal de esta ecuación diferencial permite obtener la trayectoria del centro de masas del pararrotor. Este sistema expresado en el sistema de referencia $\mathrm{T}$ toma la forma de:

$$
\frac{d}{d t}\left[\begin{array}{c}
r_{G_{1}} \\
r_{G_{2}} \\
r_{G_{3}}
\end{array}\right]=\left[\begin{array}{c}
u_{T} \\
v_{T} \\
w_{T}
\end{array}\right]=\left[\mathbf{T}_{T 1 T}\right]^{\prime}\left[\mathbf{T}_{A T 1}\right]^{\prime}\left[\begin{array}{c}
u \\
v \\
w
\end{array}\right]
$$

donde el superíndice' indica la trasposición de la matriz. Desarrollando según sus componentes resulta:

$$
\begin{gathered}
\frac{d r_{G_{1}}}{d t}=u \cos \Theta+v \sin \Theta \sin \Phi+w \sin \Theta \cos \Phi \\
\frac{d r_{G_{2}}}{d t}=v \cos \Phi-w \sin \Phi \\
\frac{d r_{G_{3}}}{d t}=-u \sin \Phi+v \cos \Theta \sin \Phi+w \cos \Theta \cos \Phi
\end{gathered}
$$




\subsection{Fuerzas sobre las palas}

En el apartado correspondiente a las fuerzas que tienen lugar sobre el cuerpo cilíndrico del pararrotor se manifiestan las que introducen las palas aplicadas sobre el punto A. En este apartado se detalla cómo están compuestas estas fuerzas, y se desarrollan las hipótesis necesarias que permiten elaborar un modelo matemático para representarlas. El desarrollo que sigue se realiza tomando como objeto de estudio a una pala genérica, que se indica mediante el subíndice $b$, siempre que sea necesario determinar algún tipo de referencia a la pala. La masa de las palas es $M_{b}$, y el tensor de inercia de las mismas, representado en el sistema $B_{b}$ respecto al punto $E_{b}$, se indica como $\mathbf{I}_{b}$, siendo la expresión correspondiente para el tensor de inercia la siguiente:

$$
\mathbf{I}_{B}=\left[\begin{array}{ccc}
I_{x_{B}} & 0 & 0 \\
0 & I_{y_{B}} & 0 \\
0 & 0 & I_{z_{B}}
\end{array}\right]
$$

La figura 10 muestra el diagrama de fuerzas y momentos sobre la pala. Las fuerzas aerodinámicas sobre las palas se originan durante la traslación y la rotación de las mismas dentro de la corriente de aire en la que se mueve el pararrotor. Estas fuerzas se suponen concentradas en el punto $P_{b}$ y se indican como $\mathbf{F}_{b}^{a}$. También se aplica, sobre el centro de masas de la pala (punto $G B_{b}$ ), el peso de la misma, indicado como $M_{b}$ g. Sobre el punto de acoplamiento $E_{b}$ aparecen fuerzas y momentos reactivos, transmitidos por el cuerpo del pararrotor, e indicados como $\mathbf{F}_{b}^{r}$ y $\mathbf{M}_{b}^{r, E_{b}}$.

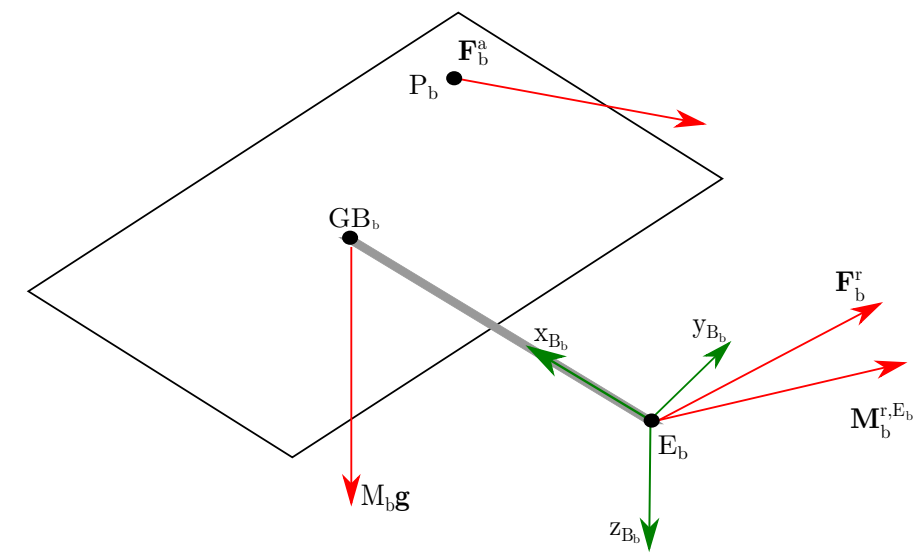

Figura 10: Diagrama de fuerzas y momentos sobre una pala del pararrotor

En la siguiente figura se muestran las fuerzas y momentos que la pala transmite al punto $E_{b}$, punto de acoplamiento entre la pala $b$ y el cuerpo del para- 
rrotor. En el diagrama se identifican las fuerzas y momentos de origen aerodinámico como así también las fuerzas transmitidas por el cuerpo del pararrotor (indicadas como reacciones mediante el superíndice r).

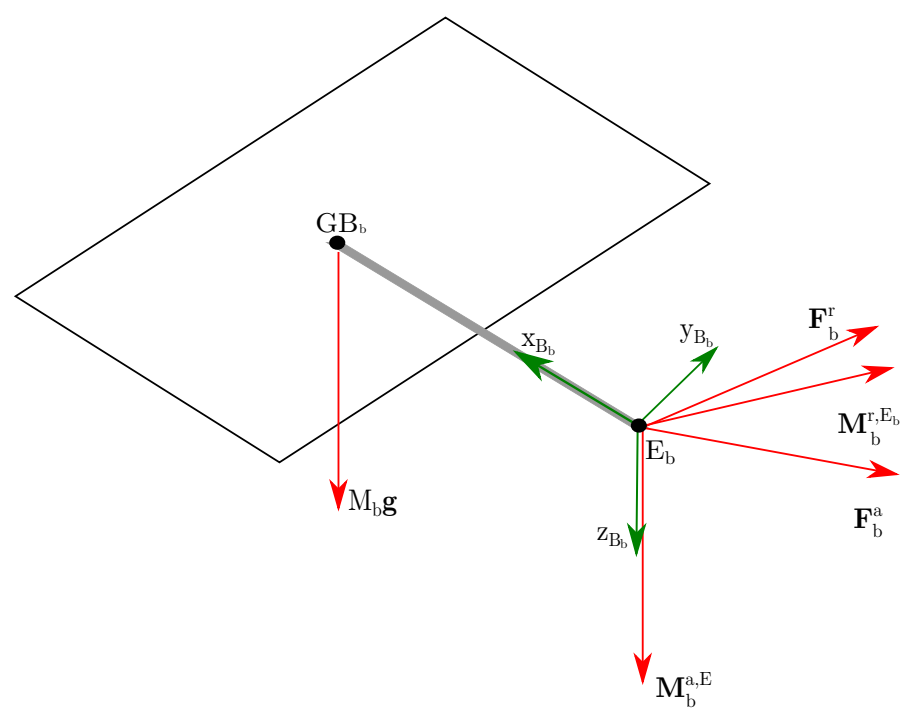

Figura 11: Diagrama de fuerzas y momentos sobre una pala del pararrotor

Para el planteo de las ecuaciones que describen la dinámica de la pala es conveniente definir diferentes magnitudes cinemáticas para una pala genérica identificada con el subíndice $b$. El acoplamiento presenta una velocidad absoluta $\mathbf{V}^{E_{b}}$. El centro de masas, indicado como $G B_{b}$, tiene una velocidad absoluta $\mathbf{V}^{G B_{b}}$. La velocidad angular de la pala en el sistema $B_{b}$ se indica como $\left.\boldsymbol{\omega}_{b}\right|_{B_{b}}$ siendo:

$$
\left.\boldsymbol{\omega}_{b}\right|_{B_{b}}=\boldsymbol{\omega}+\frac{d \theta_{b}}{d t} \mathbf{i}_{B_{b}}
$$

La velocidad del acoplamiento $E_{b}$ se expresa como sigue:

$$
\mathbf{V}^{E_{b}}=\mathbf{V}^{G}+\omega \wedge \mathbf{G E}_{b}
$$

y la velocidad del centro de masas de la pala como:

$$
\mathbf{V}^{G B_{b}}=\mathbf{V}^{E_{b}}+\left.\boldsymbol{\omega}_{b}\right|_{B_{b}} \wedge \mathbf{E}_{b} \mathbf{G B}_{b}
$$

Con la ayuda de la figura 11, se plantean las ecuaciones de Newton-Euler de manera vectorial:

$$
\mathbf{F}_{b}^{a}+\mathbf{F}_{b}^{r}+M_{b} \mathbf{g}=M_{b} \frac{d \mathbf{V}^{G B_{b}}}{d t}
$$




$$
\mathbf{M}_{b}^{a, E_{b}}+\mathbf{M}_{b}^{r, E_{b}}+\mathbf{E}_{b} \mathbf{G B}_{b} \wedge M_{b} \mathbf{g}=\frac{d \mathbf{h}_{b}}{d t}+\mathbf{E}_{b} \mathbf{G B}_{b} \wedge M_{b} \frac{d \mathbf{V}^{E_{b}}}{d t}
$$

Donde $\mathbf{M}_{b}^{a, E_{b}}=\mathbf{E}_{b} \mathbf{P}_{b} \wedge \mathbf{F}_{b}^{a}$ es el momento que realiza la fuerza aerodinámica aplicada en el centro de presiones de la pala e $\mathbf{I}_{B}$ es el tensor de inercia de la pala respecto al punto $E_{b}$ en el sistema de referencia B.

La pala transmite al punto de acoplamiento una fuerza igual en dirección y módulo pero contraria en sentido a aquella que el acoplamiento le transmite, por lo tanto se puede decir que:

$$
\mathbf{F}_{b}^{t}=-\mathbf{F}_{b}^{r}=\mathbf{F}_{b}^{a}+M_{b} \mathbf{g}-M_{b} \frac{d \mathbf{V}^{G B_{b}}}{d t}
$$

El mismo razonamiento vale para los momentos, por lo que:

$$
\mathbf{M}_{b}^{t, E_{b}}=-\mathbf{M}_{b}^{r, E_{b}}=\mathbf{M}_{b}^{a, E_{b}}+\mathbf{E}_{b} \mathbf{G B}_{b} \wedge M_{b} \mathbf{g}-\frac{d \mathbf{h}_{b}}{d t}-\mathbf{E}_{b} \mathbf{G B}_{b} \wedge M_{b} \frac{d \mathbf{V}^{E_{b}}}{d t}
$$

Se puede redefinir a la fuerza gravitatoria de la pala como:

$$
\mathbf{F}_{b}^{g}=M_{b} \mathbf{g}
$$

y el término inercial como:

$$
\mathbf{F}_{b}^{i}=-M_{p} \frac{d \mathbf{V}^{G B_{b}}}{d t}
$$

En los momentos involucrados en la expresión (26) se puede identificar el que genera la fuerza gravitatoria y redefinirlo como:

$$
\mathbf{M}^{g, E_{b}}=\mathbf{E}_{b} \mathbf{G B}_{b} \wedge F_{b}^{g}
$$

y el momento inercial como:

$$
\mathbf{M}^{i, E_{b}}=-\frac{d \mathbf{h}_{b}}{d t}-M_{b} \mathbf{E}_{b} \mathbf{G B}_{b} \wedge \frac{d \mathbf{V}^{G B_{b}}}{d t}
$$

En base a estas redefiniciones, las expresiones (25) y (26) pueden reformularse de manera compacta como:

$$
\mathbf{F}_{b}^{r}+\mathbf{F}_{b}^{a}+\mathbf{F}_{b}^{g}+\mathbf{F}_{b}^{i}=0
$$




$$
\mathbf{M}_{b}^{r, E_{b}}+\mathbf{M}_{b}^{a, E_{b}}+\mathbf{M}_{b}^{g, E_{b}}+\mathbf{M}_{b}^{i, E_{b}}=0
$$

El sistema (27) da cuenta de la magnitud de los efectos que las palas transmiten al cuerpo cilíndrico a través del punto Eb.

\subsubsection{Leyes de variación de paso}

El ángulo de paso que se le asigna a cada pala se constituye a partir de la definición de una función armónica de primer orden, del tipo:

$$
\theta_{b}(\psi)=\theta_{0}(t)+\theta_{1 C}(t) \cos [\psi(t)+(b-1) \pi]+\theta_{1 S}(t) \sin [\psi(t)+(b-1) \pi]
$$

donde $\psi_{b}(t)=\psi(t)+(b-1) \pi$. En la ecuación (28) la función $\theta_{0}(t)$ se denomina paso colectivo, que es el asignado a todas las palas de manera simultánea. Las funciones $\theta_{1 C}(t)$ y $\theta_{1 S}(t)$ son los ángulos de paso cíclico lateral y longitudinal respectivamente, y son quienes dominan la actitud en los planos correspondientes. Estos tres parámetros son los elementos del vector de control $\mathbf{u}_{C}$.

La derivada primera respecto al tiempo de la ecuación (28) es:

$$
\frac{d \theta_{b}}{d t}=\frac{d \theta_{0}}{d t}+\frac{d \theta_{1 C}}{d t} \cos \psi_{b}+\frac{d \theta_{1 S}}{d t} \sin \psi_{b}+\left(-\sin \psi_{b} \theta_{1 C}+\cos \psi_{b} \theta_{1 S}\right) \frac{d \psi}{d t}
$$

Del mismo modo se puede definir la derivada segunda de la ecuación (28) respecto al tiempo:

$$
\begin{aligned}
\frac{d^{2} \theta_{b}}{d t^{2}} & =\frac{d^{2} \theta_{0}}{d t^{2}}+\frac{d^{2} \theta_{1 C}}{d t^{2}} \cos \psi_{b}+\frac{d^{2} \theta_{1 S}}{d t^{2}} \sin \psi_{b}-2 \frac{d \theta_{1 C}}{d t} \frac{d \psi}{d t} \sin \psi_{b}+2 \frac{d \theta_{1 S}}{d t} \frac{d \psi}{d t} \cos \psi_{b}- \\
& -\theta_{1 C}\left[\frac{d^{2} \psi}{d t^{2}} \sin \psi_{b}+\left(\frac{d \psi}{d t}\right)^{2} \cos \psi_{b}\right]+\theta_{1 S}\left[\frac{d^{2} \psi}{d t^{2}} \cos \psi_{b}-\left(\frac{d \psi}{d t}\right)^{2} \sin \psi_{b}\right]
\end{aligned}
$$

\subsection{Forma canónica de la ecuación de estado del pararrotor}

En virtud del desarrollo previo, se formula la representación del sistema matemático en su forma canónica. Esto permite describir el problema matemático de manera directa para su resolución mediante la implementación de un código 
numérico. Las ecuaciones diferenciales ordinarias no lineales (8), (9), (12) y (17) se expresan en función del vector de estado y el vector de control.

En base a las definiciones previas, el vector de estado se constituye como sigue:

$$
\mathbf{x}(t)=\left[\begin{array}{c}
u \\
v \\
w \\
\omega_{x} \\
\omega_{y} \\
\omega_{z} \\
\Theta \\
\Phi \\
\psi \\
r_{G 1} \\
r_{G 2} \\
r_{G 3}
\end{array}\right]
$$

y el vector de control como:

$$
\mathbf{u}_{C}(t)=\left[\begin{array}{c}
\theta_{0} \\
\theta_{1 S} \\
\theta_{1 C}
\end{array}\right]
$$

La relación entre el vector de estado y el vector de control viene dada por:

$$
\mathbf{M} \frac{d \mathbf{x}(t)}{d t}=\mathbf{f}
$$

donde $\mathbf{M}$ es una matriz cuadrada de 12 x 12 y f un vector fila de 12 elementos. A su vez, son funciones no lineales dependientes de $\mathbf{x}(t)$ y de $\mathbf{u}_{C}(t)$ y sus derivadas. Dentro de cada una de estas matrices es conveniente identificar cada uno de los subsistemas que las conforman. $\left[\mathbf{M}^{T R}\right]$.

La matriz $\mathbf{M}$ está formada por las submatrices $\left[\mathbf{M}^{F}\right],\left[\mathbf{M}^{M}\right],\left[\mathbf{M}^{L C}\right] \mathrm{y}$

La submatriz $\left[\mathbf{M}^{F}\right]$ es aquella asociada a las fuerzas inerciales del cilindro del pararrotor y sus palas, es decir:

$$
\left[\mathbf{M}^{F}\right]=\left[\mathbf{M}_{H}^{F}\right]+\left[\mathbf{M}_{B}^{F}\right]
$$


siendo $\left[\mathbf{M}_{H}^{F}\right]$ la matriz asociada a las fuerzas inerciales del cilindro y $\left[\mathbf{M}_{B}^{F}\right]$ asociada a la fuerzas inerciales de las de las palas.

La submatriz $\left[\mathbf{M}^{M}\right]$ es la asociada a los momentos inerciales del cuerpo del pararrotor y de las palas alrededor del centro de masas del cuerpo pararrotor. Esta submatriz puede desglosarse en:

$$
\left[\mathbf{M}^{M}\right]=\left[\mathbf{M}_{H}^{M}\right]+\left[\mathbf{M}_{B}^{M}\right]
$$

La submatriz $\left[\mathbf{M}^{L C}\right]$ es la que representa a la ligadura cinemática impuesta, y la submatriz $\left[\mathbf{M}^{T R}\right]$ corresponde a la trayectoria del pararrotor expresada en ejes tierra.

En lo que sigue se desarrolla la obtención de cada uno de los elementos de estas matrices.

\subsubsection{Forma canónica de la ecuación de Newton}

En este apartado, se desarrollan las expresiones de la submatriz del sistema y del vector independiente para las fuerzas del cilindro y las palas. La dimensión de las submatrices del sistema $\mathbf{M}_{H}^{F}$ y $\mathbf{M}_{B}^{F}$ es $3 \times 12$, mientras que los vectores independientes $\mathbf{f}_{H}^{F}$ y $\mathbf{f}_{B}^{F}$ resultan de dimensión 3x1. La ecuación (8) puede transformarse a la forma canónica considerando que el término inercial puede expresarse como:

$$
\mathbf{F}_{H}^{i}=\left[\mathbf{M}_{H}^{F}\right] \frac{d \mathbf{x}}{d t}-\mathbf{f}_{H}^{F}
$$

donde $\mathbf{M}_{H}^{F}$ y $\mathbf{f}_{H}^{F}$ son funciones no lineales del vector de estado y el vector de control. Asimismo, el término inercial de las fuerzas transmitidas por las palas (ecuación (27) ) puede transformarse como sigue:

$$
\mathbf{F}^{i}=\left[\mathbf{M}_{B}^{F}\right] \frac{d \mathbf{x}}{d t}-\mathbf{f}_{B}^{F}
$$

La ecuación (8) se reescribe en su forma canónica como sigue:

$$
\left[\mathbf{M}^{F}\right] \frac{d \mathbf{x}}{d t}=\mathbf{f}^{F}
$$

donde se puede identificar que:

$$
\left[\mathbf{M}^{F}\right]=\left[\mathbf{M}_{H}^{F}\right]+\left[\mathbf{M}_{B}^{F}\right]
$$




$$
\mathbf{f}^{F}=\mathbf{f}_{B}^{F}+\mathbf{f}_{H}^{F}-\mathbf{F}_{H}^{a}-\mathbf{F}_{H}^{g}-\mathbf{F}^{a}-\mathbf{F}^{g}
$$

\subsubsection{Forma canónica de la ecuación de Euler}

A continuación, se desarrollan las submatrices y los vectores correspondientes a los momentos que generan el cilindro y las palas. Del mismo modo que para la ecuación de Newton, se puede reescribir la ecuación (9) considerando el término inercial como:

$$
\mathbf{M}_{H}^{i, G}=\left[\mathbf{M}_{H}^{M}\right] \frac{d \mathbf{x}}{d t}-\mathbf{f}_{H}^{M}
$$

Donde $\mathbf{M}_{H}^{M}$ y $\mathbf{f}_{H}^{M}$ son de dimensión 3 x 12 y 3 x 1 respectivamente. El momento transmitido por las palas en el punto $\mathrm{G}$ (centro de masas del pararrotor) tiene una componente inercial que puede escribirse como:

$$
\mathbf{M}_{B}^{i, G}+\mathbf{G A} \wedge \mathbf{F}^{i}=\left[\mathbf{M}_{B}^{M}\right] \frac{d \mathbf{x}}{d t}-\mathbf{f}_{B}^{M}
$$

Aquí también, las matrices $\mathbf{M}_{B}^{M}$ y $\mathbf{f}_{B}^{M}$ tienen dimensión 3 x 12 y 3 x 1 respectivamente. Sustituyendo en la ecuación (9) se tiene la ecuación de Euler en su forma canónica:

$$
\left[\mathbf{M}^{M}\right] \frac{d \mathbf{x}}{d t}=\mathbf{f}^{M}
$$

donde

$$
\left[\mathbf{M}^{M}\right]=\left[\mathbf{M}_{H}^{M}\right]+\left[\mathbf{M}_{B}^{M}\right]
$$

$\mathrm{y}$

$$
\mathbf{f}^{M}=\mathbf{f}_{H}^{M}+\mathbf{f}_{B}^{M}-\mathbf{M}_{H}^{a, G}-\mathbf{M}^{a, A}-\mathbf{M}^{g, A}-\mathbf{G A} \wedge\left(\mathbf{F}^{a}+\mathbf{F}^{g}\right)
$$

\subsubsection{Forma canónica de la ligadura cinemática y la ecuación de trayec- toria}

En el caso de la ligadura cinemática, la expresión en la forma canónica viene dada por la ecuación (12), que se expresa del modo siguiente: 


$$
\left[\mathbf{M}^{L C}\right] \frac{d \mathbf{x}_{7: 9 \times 1}}{d t}=\mathbf{f}^{L C}
$$

en donde $\mathbf{x}_{7: 9 \times 1}=[\Theta, \Phi, \psi]^{\prime}$ y $\mathbf{f}^{L C}=\mathbf{f}_{3 \times 1}^{L C}=\left[\omega_{x}, \omega_{y}, \omega_{x}\right]^{\prime}$. La matriz $\left[\mathbf{M}^{L C}\right]$ es una matriz cuadrada de 3 x 3 y viene dada por la ecuación (13):

$$
\mathbf{L C}=\left[\begin{array}{ccc}
0 & 1 & 0 \\
\cos \Phi & 0 & 0 \\
\sin \Phi & 0 & 1
\end{array}\right]
$$

La expresión en forma canónica de la trayectoria viene dada por:

$$
\left[\mathbf{M}^{T R}\right]=\frac{d \mathbf{x}_{10: 12 \times 1}}{d t}-\mathbf{f}^{T R}
$$

donde $\mathbf{M}^{T R}$ es una matriz cuadrada de dimensión $3 \times 3, \mathbf{x}_{10: 12 \times 1}=\left[r_{G 1}, r_{G 2}, r_{G 3}\right]^{\prime} \mathrm{y}$ $\mathbf{f}^{T R}$ es un vector de dimensión $3 \times 1$ que se obtiene del tercer miembro de la ecuación (17).

\subsubsection{Ecuación de estado en forma canónica}

Para concluir esta sección se presenta la ecuación de estado en su forma canónica, representada de modo de facilitar la resolución del sistema de ecuaciones diferenciales obtenido. Cada subsistema desarrollado previamente se integra al modelo general del pararrotor, ubicando cada una de las submatrices en el lugar correspondiente. De este modo se tiene que:

$$
\left[\mathbf{M}_{12 \times 12}\right]=\left[\begin{array}{c}
{\left[\mathbf{M}_{3 \times 12}^{F}\right]} \\
{\left[\mathbf{M}_{3 \times 12}^{M}\right]} \\
{\left[\mathbf{0}_{3 \times 6}\right]\left[\mathbf{M}_{3 \times 3}^{L C}\right]\left[\mathbf{0}_{3 \times 3}\right]} \\
{\left[\mathbf{0}_{3 \times 9}\right]\left[\mathbf{M}_{3 \times 3}^{T R}\right]}
\end{array}\right]
$$

y:

$$
\mathbf{f}_{12 \times 1}=\left[\begin{array}{c}
\mathbf{f}_{3 \times 1}^{F} \\
\mathbf{f}_{3 \times 1}^{M} \\
\mathbf{f}_{3 \times 1}^{L C} \\
\mathbf{f}_{3 \times 1}^{T R}
\end{array}\right]
$$

Esta formulación permite describir genéricamente el sistema de ecuaciones diferenciales ordinarias no lineales. Por ejemplo, el efecto de las fuerzas y momentos de origen inercial, tanto de las palas como del cuerpo cilíndrico, aparecen 
en los primeros 3x6 elementos de la matriz de masas, que son los que constituyen las submatrices $\mathbf{M}^{F}$ y $\mathbf{M}^{H}$. En el vector $\mathbf{f}$, se manifiestan a través de las primeras seis componentes. Los efectos aerodinámicos interactúan junto a los inerciales a través de las componentes del vector $\mathbf{f}$.

Cada uno de los elementos que constituyen la matriz (39) y el vector (40) se obtienen analítica y numéricamente en los apartados que siguen. Sustituyendo en el sistema (33) con condiciones iniciales $\mathbf{x}_{12 \times 1}=\mathbf{x}(0)$ y con una ley de control $\mathbf{u}_{C}(t)$ conocida se puede describir la evolución de cada una de las variables que conforman el vector de estado y la determinación de la trayectoria del pararrotor. Desde ya que, para su utilización con fines prácticos, las condiciones iniciales y el vector de control seleccionados deben ser tales que garanticen que el sistema (33) tenga una solución convergente. A tal fin, es necesario indagar alternativas en la resolución del sistema:

$$
\mathbf{M}_{12 \times 12} \frac{d \mathbf{x}(t)}{d t}=\mathbf{f}_{12 \times 1}
$$

\subsection{Solución de la ecuación de estado del pararrotor}

Para la solución general de la ecuación de estado que caracteriza el movimiento de un pararrotor en vuelo libre (ecuación (41)), se necesita simbolizar mediante expresiones matemáticas cada uno de los elementos que constituyen la matriz de masas y el término independiente del sistema citado. A tal efecto, se estudia cada uno de los elementos y se obtiene su representación matemática, para ser utilizados en la resolución del sistema. Se desarrollan en primer lugar los términos de origen inercial, e inmediatamente después los que tienen origen aerodinámico.

\subsubsection{Cálculo de fuerzas y momentos de origen inercial}

Para el desarrollo de la expresión de momentos se considera lo desarrollado en [27]. Lo que se busca en este apartado es determinar expresiones matemáticas para las fuerzas y el momento transmitido al pararrotor debido a efectos inerciales durante el cambio en el ángulo de paso $\theta_{b}$ en vacío (no se incluyen los efectos aerodinámicos). En términos elementales, este fenómeno puede explicarse de forma conceptual con la ayuda de la figura 12. Allí se observa que en el plano $x_{A 1 b}-y_{A 1 b}$ las fuerzas centrífugas que actúan sobre las masas elementales dispuestas en el borde de ataque y el borde de fuga del perfil se encuentran equilibradas, mientras que en el plano $z_{A 1 b}-y_{A 1 b}$, las rectas de acción de las fuerzas no coinciden, por lo que se genera una cupla que se transmite al eje del rotor. 

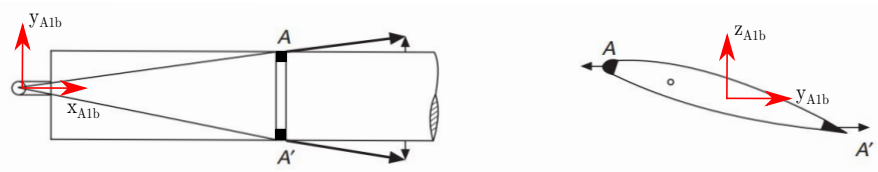

Figura 12: Generación del momento inercial debido al desbalance de masas

La determinación de las acciones inerciales transmitidas consiste en asignar a cada uno de los términos del sistema (27) una expresión en función de las variables de estado. La siguiente figura muestra las principales referencias dinámicas y cinemáticas que ayudan al planteo:

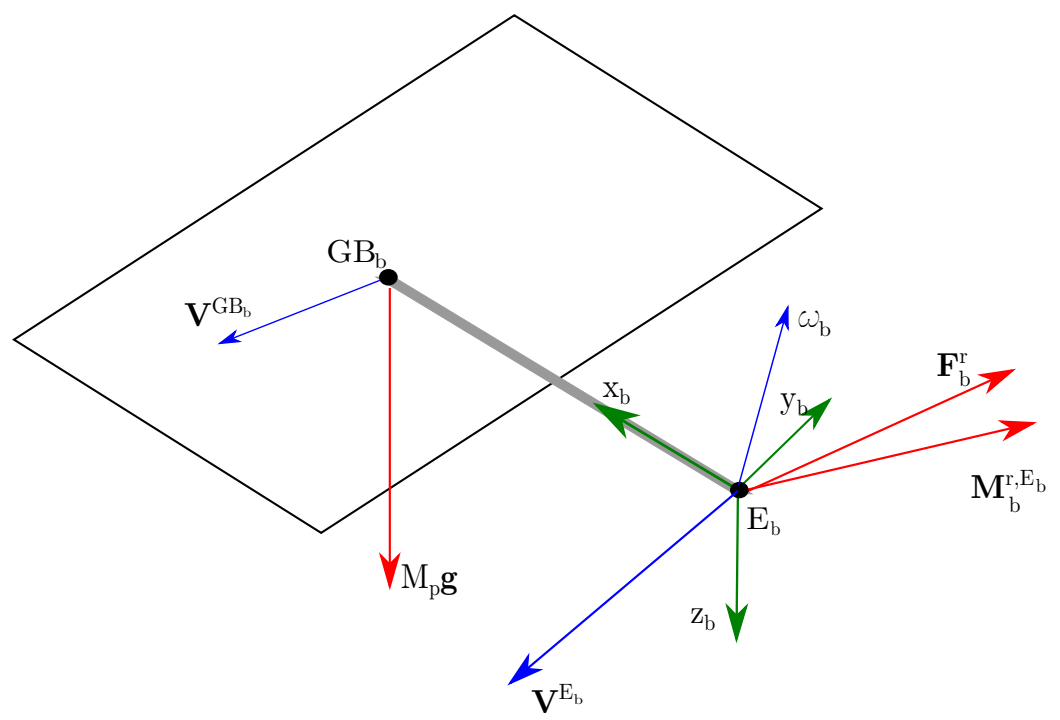

Figura 13: Referencias dinámicas y cinemáticas para el cálculo de acciones inerciales de las palas

En la figura 13 se identifican como variables cinemáticas a la velocidad del centro de masas de la pala $\left(\mathbf{V}^{G B_{b}}\right)$, la velocidad del punto de acoplamiento $E_{b}$ $\left(\mathbf{V}^{E_{b}}\right)$ y la velocidad angular de pala $\left(\boldsymbol{\omega}_{b}\right)$. Las fuerzas inerciales que se manifiestan son el peso de la pala $\left(M_{b} \mathbf{g}\right)$, y las acciones que el cuerpo del pararrotor transmite a la pala a través del punto de acoplamiento (la fuerza $\mathbf{F}_{b}^{r}$ y el momento $\mathbf{M}_{b}^{r, E_{b}}$ ). La expresión de la fuerza inercial que las palas transmiten al cuerpo del pararrotor se obtiene utilizando la formulación del término inercial utilizada en la ecuación canónica de Newton (ecuación (36)). Para esto, se debe considerar a la aceleración del centro de masas de la pala como:

$$
\mathbf{a}^{G B_{b}}=\frac{d \mathbf{V}^{G B_{b}}}{d t}
$$


y la fuerza es :

$$
\mathbf{F}_{b}^{i}=-M_{b} \cdot \mathbf{a}^{G B_{b}}
$$

La determinación de la aceleración del centro de masas se produce a partir de la derivada de la velocidad del centro de masas de la pala. La expresión que modela la velocidad del centro de masas está dada por:

$$
\mathbf{V}^{G B_{b}}=\mathbf{V}^{E_{b}}+\boldsymbol{\omega}_{b} \wedge \mathbf{E}_{b} \mathbf{G B}_{b}
$$

Se puede expresar esta velocidad en el sistema de referencia A como sigue:

$$
\left.\mathbf{V}^{G B_{b}}\right|_{A}=\left.\left[\mathbf{T}_{A A 1 b}\right]\left[\mathbf{T}_{A 1 b B b}\right] \mathbf{V}^{G B_{b}}\right|_{B_{b}}
$$

y al derivarse respecto al tiempo en el sistema A resulta la aceleración del centro de masas.

La velocidad angular de la pala es:

$$
\boldsymbol{\omega}_{b}=\frac{d \theta_{b}}{d t} \mathbf{i}_{B}+\omega_{x} \mathbf{i}_{A}+\omega_{y} \mathbf{j}_{A}+\omega_{z} \mathbf{k}_{A}
$$

El momento cinético de la pala se obiene multiplicando el tensor de inercia en el sistema $B_{b}$ por la velocidad angular $\boldsymbol{\omega}$ expresada en el sistema de referencia de la pala:

$$
\left.\mathbf{h}_{b}\right|_{B_{b}}=\left.\left[\mathbf{I}_{b}\right] \boldsymbol{\omega}_{b}\right|_{B_{b}}
$$

El momento inercial, en el sistema de referencia de las palas, es igual (en valor absoluto) a la derivada absoluta del momento cinético de las mismas:

$$
\mathbf{M}_{B_{b}}^{i, E_{b}}=-\frac{d \mathbf{h}_{b}}{d t}
$$

La derivada absoluta del momento cinético $\left.\mathbf{h}_{b}\right|_{B_{b}}$ se calcula mediante:

$$
\frac{d \mathbf{h}_{b}}{d t}=\left(\frac{\left.d \mathbf{h}_{b}\right|_{B_{b}}}{d t}\right)+\left.\boldsymbol{\omega}_{b} \wedge \mathbf{h}_{b}\right|_{B_{b}}
$$

La transformación de las expresiones anteriores al sistema de referencia A se realiza utilizando las correspondientes matrices de transformación (3.3), es decir: 


$$
\mathbf{M}_{A}^{t, E_{b}}=\left[\mathbf{T}_{A A 1 b}\right]\left[\mathbf{T}_{A 1 b B_{b}}\right] \mathbf{M}_{B}^{t, E_{b}}
$$

donde $\left[\mathbf{T}_{A A 1 b}\right]=\left[\mathbf{T}_{A 1 b A}\right]^{\prime}$. Al ejecutar la transformación (50) obtenemos el conjunto de ecuaciones que dan los momentos en el sistema A.

\subsubsection{Cálculo de fuerzas y momentos de origen aerodinámico}

Las fuerzas y momentos de origen aerodinámico son aquellos generados por el movimiento relativo del fluido sobre las palas. El fenómeno sobre el que se desarrolla el presente trabajo se da cuando el pararrotor se encuentra en régimen de rotación, de manera que se establece una corriente fluida sobre la pala que provoca la aparición de la sustentación y la resistencia. Cabe aclarar que también aparecen momentos aerodinámicos sin la necesidad de que se establezca un régimen de rotación en el pararrotor, aunque no es objeto de estudio en este trabajo. En este apartado se busca obtener expresiones simbólicas para las fuerzas y momentos aerodinámicos que generan las palas, suponiendo las fuerzas aplicadas en el centro de presiones de la pala $\left(P_{b}\right)$.

La figura siguiente muestra una vista lateral de una pala, con los sistemas de referencia utilizados, las fuerzas y velocidades que aparecen sobre las mismas.

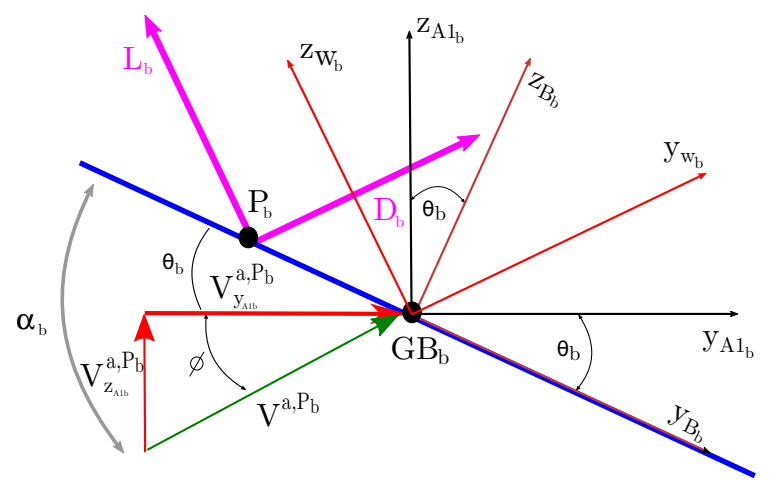

Figura 14: Diagrama de fuerzas sobre la pala.

En la figura 14, las fuerzas de resistencia y sustentación $\left(\mathbf{L}_{b}\right.$ y $\left.\mathbf{D}_{b}\right)$ están aplicadas en el centro de presiones de la pala (punto $P_{b}$ ). Las componentes de la velocidad del viento relativa a la pala se indican cómo $V_{y_{B b}}^{a, P_{b}}$ y $V_{z_{B b}}^{a, P_{b}}$, y la relación entre ambas determina el ángulo de incidencia de flujo $\phi$. El ángulo de ataque de la pala, $\alpha_{b}$, queda definido una vez establecido el ángulo de paso de la pala $\left(\theta_{b}\right)$. Con esta convención, el ángulo de paso de la pala $\left(\theta_{b}\right)$ aumenta en sentido antihorario. 


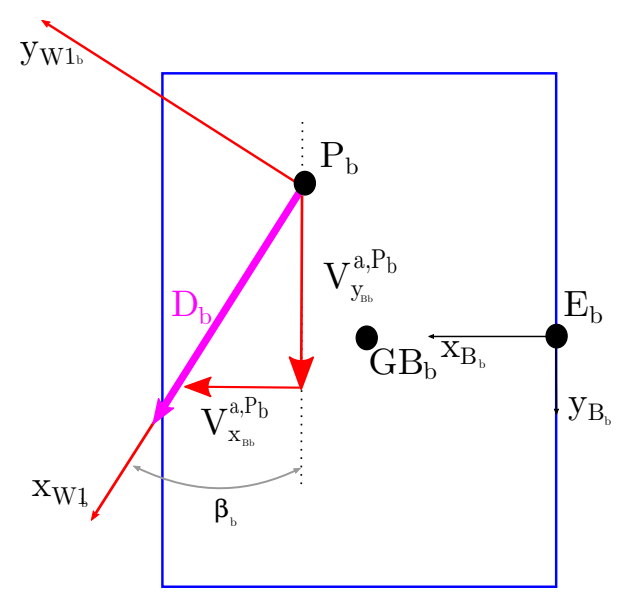

Figura 15: Vista en planta del diagrama de fuerzas sobre la pala.

En el caso bajo estudio debe considerarse que, debido a que las palas tienen un movimiento cíclico del ángulo de paso, existe una componente de velocidad angular según el eje $x_{B_{b}}$ que genera una velocidad relativa extra en el centro de presiones.

De esta manera se tiene que la velocidad del centro de presiones de la pala viene dada por la siguiente expresión:

$$
\mathbf{V}^{a, P_{b}}=-\left(\mathbf{V}^{E_{b}}+\left.\boldsymbol{\omega}_{b}\right|_{B_{b}} \wedge \mathbf{E}_{b} \mathbf{P}_{b}\right)+\mathbf{U}_{W}+\mathbf{v}_{i}
$$

donde

$$
\mathbf{V}^{E_{b}}=\mathbf{V}^{G}+\boldsymbol{\omega} \wedge \mathbf{G E}_{b}
$$

Los términos $\mathbf{U}_{W}$ y $\mathbf{v}_{i}$ representan la velocidad del viento y la velocidad inducida por el rotor respectivamente. El término entre paréntesis es el que corresponde al movimiento del centro de presiones.

La velocidad $\left.\boldsymbol{\omega}_{b}\right|_{B_{b}}$ es la velocidad angular del pararrotor expresada en ejes $B_{b}$. Dicha formulación se obtiene valiéndose de las matrices de transformación $\left[\mathbf{T}_{A 1 b A}\right]$ y $\left[\mathbf{T}_{A 1 b B_{b}}\right]$ ( matrices 3.3 y 3.3 ).

Se puede expresar la velocidad del aire respecto al punto $P_{b}$ en términos de sus componentes según el sistema $B_{b}$ :

$$
\mathbf{V}^{a, P_{b}}=V_{x_{B_{b}}}^{a, P_{b}} \mathbf{i}_{B_{b}}+V_{y_{B_{b}}}^{a, P_{b}} \mathbf{j}_{B_{b}}+V_{z_{B_{b}}}^{a, P_{b}} \mathbf{k}_{B_{b}}
$$


y a partir de esta descomposición se puede obtener la formulación de los ángulos de ataque $\left(\alpha_{b}\right)$ y deslizamiento $\left(\beta_{b}\right)$ indicados en las figuras 14 y 15 :

$$
\begin{gathered}
\alpha_{b}=\arctan \left(\frac{-V_{z_{B_{b}}}^{P_{b}}}{\sqrt{\left(V_{x_{B_{b}}}^{P_{b}}\right)^{2}+\left(V_{y_{B_{b}}}^{P_{b}}\right)^{2}}}\right) \\
\beta_{b}=\arctan \left(\frac{V_{x_{B_{b}}}^{P_{b}}}{V_{y_{B_{b}}}^{P_{b}}}\right)
\end{gathered}
$$

Con la definición de estos ángulos se pueden establecer un par de sistemas de referencia según las componentes de la velocidad del viento, que relacionan los ángulos de ataque y deslizamiento de las palas (ángulos $\alpha_{b}$ y $\beta_{b}$ ) con las direcciones definidas por el sistema $B_{b}$. Estas transformaciones son:

$$
\left[\mathbf{T}_{B_{b} W 1 b}\right]=\left[\begin{array}{ccc}
\sin \beta_{b} & -\cos \beta_{b} & 0 \\
-\cos \beta_{b} & -\sin \beta_{b} & 0 \\
0 & 0 & 1
\end{array}\right]
$$

Matriz de transformación del sistema W1b al Bb

$$
\left[\mathbf{T}_{W 1 b W b}\right]=\left[\begin{array}{ccc}
\cos \alpha_{b} & 0 & \sin \alpha_{b} \\
0 & 1 & 0 \\
\sin \alpha_{b} & 0 & \cos \alpha_{b}
\end{array}\right]
$$

Matriz de transformación del sistema Wb al W1b

Con estos sistemas de referencia se pueden plantear las fuerzas aerodinámicas de las palas como:

$$
\mathbf{F}_{b}^{a}=D_{b} \mathbf{i}_{W b}+Y_{b} \mathbf{j}_{W b}+L_{b} \mathbf{k}_{W b}
$$

La fuerza de sustentación tiene la dirección $\mathbf{k}_{W b}$, mientras que la resistencia está direccionada según $\mathbf{i}_{W b}$. La componente en la dirección $\mathbf{j}_{W b}$ se considera nula, asumiendo la hipótesis de que el flujo a través de la pala es bidimensional. Esto se debe a que, a lo largo del eje $\mathbf{j}_{W b}$, la componente aerodinámica es de menor 
orden que en las otras direcciones, y tiene un comportamiento cíclico alrededor de un valor promedio nulo [29].

Generalizando el desarrollo de la tesis de Piechocki [21], se pueden estimar las fuerzas aerodinámicas partiendo de las siguientes expresiones:

$$
\begin{aligned}
L_{b} & =\frac{1}{2} \rho S C_{L_{b}}\left(\alpha_{b}, \beta_{b}\right)\left|U_{r}\right|^{2} \\
D_{b} & =\frac{1}{2} \rho S C_{D}\left(\alpha_{b}, \beta_{b}\right)\left|U_{r}\right|^{2}
\end{aligned}
$$

donde

- $\rho$ es la densidad del aire,

- $C_{L_{b}}\left(\alpha_{b}\right)$ es el coeficiente de sustentación de la pala $b$,

- $C_{D}$ es el coeficiente de resistencia de la pala,

- $\alpha_{b}$ es el ángulo de ataque de la pala $b$,

- $\beta_{b}$ es el ángulo de deslizamiento de la pala $b$,

- $U_{r}=\left|\mathbf{V}^{P_{b}}\right|$ es el módulo de la velocidad relativa a la pala,

- $S$ es la superficie de la pala.

La fuerza aerodinámica resultante sobre cada pala en ejes [B] resulta:

$$
\left.\mathbf{F}_{b}^{a}\right|_{B}=\left[\mathbf{T}_{B_{b} W 1 b}\right]\left[\mathbf{T}_{W 1 b W b}\right]\left[\begin{array}{c}
D_{b} \\
0 \\
L_{b}
\end{array}\right]
$$

El cálculo del torque se realiza por medio del producto vectorial entre el vector posición del centro de presiones y el vector fuerza aerodinámica resultante. El momento instantáneo de las fuerzas se calcula como el producto $\mathbf{M}_{b}^{a, E_{b}}=\mathbf{E}_{b} \mathbf{P}_{b} \wedge \mathbf{F}_{b}^{a}$, donde $\mathbf{E}_{b} \mathbf{P}_{b}$ es el vector posición del centro de presiones de las palas respecto al punto de acoplamiento de la pala $b$ (punto $E_{b}$ ). 


\subsubsection{Velocidad del viento}

La variable $\mathbf{U}_{W}$, presentada en la ecuación (51), representa la velocidad del viento atmosférico. En general, su magnitud será conocida en el sistema de referencia tierra. La variable $\mathbf{U}_{W}$ se define cómo:

$$
\mathbf{U}_{W}=u_{1 T} \mathbf{i}_{T}+u_{2 T} \mathbf{j}_{T}+u_{3 T} \mathbf{k}_{T}
$$

La expresión de $\mathbf{U}_{W}$ en el sistema de referencia asociado a las palas se obtiene aplicando la transformación correspondiente entre los sistemas de referencia. Por ejemplo, para conocer la expresión en el sistema de referencia de la pala $B_{b}$ se premultiplica el vector como se muestra a continuación:

$$
\left.\mathbf{U}_{W}\right|_{B_{b}}=\left[\mathbf{T}_{B_{b} A 1 b}\right]\left[\mathbf{T}_{A 1 b A}\right]\left[\mathbf{T}_{A T 1}\right]\left[\mathbf{T}_{T 1 T}\right]\left[\begin{array}{l}
u_{1 T} \\
u_{2 T} \\
u_{3 T}
\end{array}\right]
$$

\subsubsection{Velocidad inducida}

Debido a la generación de tracción por parte de las palas del pararrotor, existe una variación en la cantidad de movimiento del flujo que lo atraviesa. Esto implica la aparición de una velocidad inducida en el flujo.

Existen distintos estados aerodinámicos en los que puede hallarse sumergido un rotor, que se encuentran desarrollados en la bibliografía de referencia [27], [30], [29]. Para el caso en estudio, puede suponerse que la relación entre la velocidad de descenso, $\mathbf{U}_{W}$, y la velocidad inducida, $\mathbf{v}_{i}$, es mayor, en valor absoluto, a 2 . Este caso se conoce como molinete frenante. En esta situación, la estela del rotor se encuentra bien definida, sobre la zona superior del mismo.

Para el presente desarrollo se trabaja con la hipótesis de no considerar la velocidad inducida, dado que es dos veces más pequeña que la velocidad de descenso. Por lo tanto, no introduce modificaciones sustanciales en el régimen aerodinámico que se establece a través del pararrotor. Esto significa asignar, en la ecuación (51), $\mathbf{v}_{i}=0$.

\subsubsection{Resolución del sistema de ecuaciones}

La implementación del modelo teórico elaborado en un código de resolución numérica permite obtener resultados de las magnitudes que caracterizan el 
estado del sistema, como así también representaciones del comportamiento del dispositivo en distintas configuraciones de vuelo.

El desarrollo se realiza mediante una herramienta de cálculo simbólico (Maple ( $)$ ), de la que se obtienen los elementos que constituyen la matriz $\mathbf{M}_{12 \times 12} \mathrm{y}$ el vector $\mathbf{f}_{12 \times 12}$. Las expresiones obtenidas son las que se utilizan en la formulación numérica a partir de la cuál se obtienen las soluciones, para un vector de estado inicial $\mathbf{x}(0)$ y un vector de control $\mathbf{u}_{C}$ definidos.

\subsection{Modelo del pararrotor en túnel de viento}

A continuación, se modela el problema de la dinámica del pararrotor en el túnel de viento, determinando las fuerzas y momentos con las restricciones que impone el montaje del pararrotor en el túnel de viento. La figura 16 muestra el esquema del montaje experimental del pararrotor, donde se indican los sistemas de referencia que se utilizan:

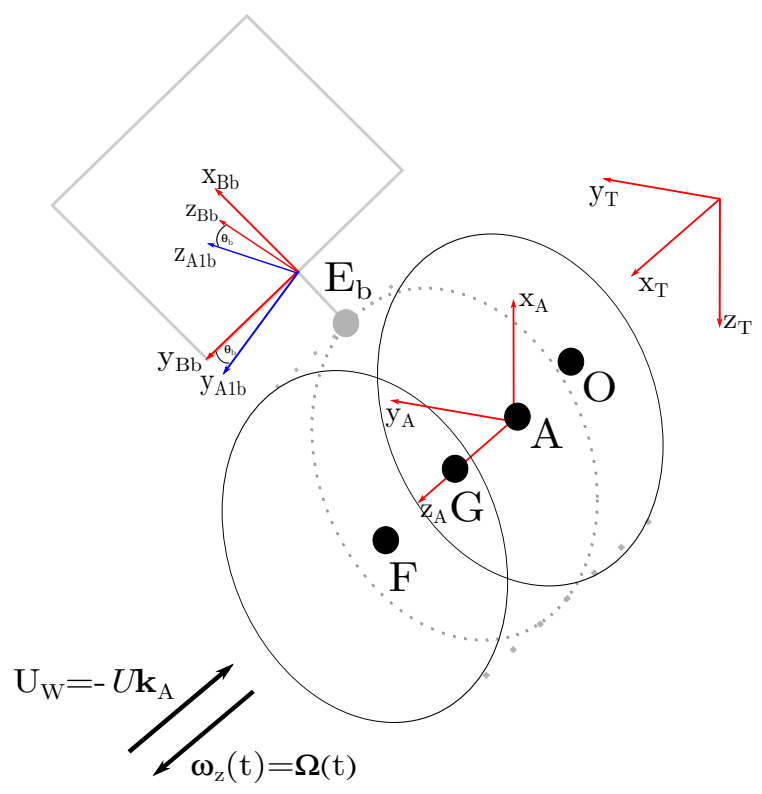

Figura 16: Esquema de montaje del pararrotor en el túnel de viento

Como se menciona anteriormente, el torque aerodinámico que transmiten cada pala al cuerpo del pararrotor a través del punto $E_{b}$ se obtiene realizando el siguiente producto:

$$
\mathbf{M}_{b_{B}}^{a, E_{b}}=\mathbf{E}_{b} \mathbf{P}_{b} \wedge \mathbf{F}_{b}^{a}
$$


Mientras que el inercial viene dado por:

$$
\mathbf{M}_{b_{B}}^{i, E_{b}}=-\frac{d \mathbf{h}_{b}}{d t}-M_{b} \mathbf{E}_{b} \mathbf{G B}_{b} \wedge \frac{d \mathbf{V}^{G B_{b}}}{d t}
$$

Se considera cómo hipótesis que las acciones aerodinámicas directas sobre el cuerpo del pararrotor son despreciables, resultando que $\mathbf{M}_{H}^{a, F}=\mathbf{F}_{H}^{a}=0$.

Tal como se define en la ecuación (6), la vinculación entre los efectos inerciales y aerodinámicos viene dada por la ecuación de conservación de momento angular del pararrotor.

Se particulariza esta ecuación para el modelo de pararrotor en túnel de viento, atendendiendo a las restricciones impartidas por el montaje del dispositivo, con el objetivo de validar los resultados con los ensayos en el túnel aerodinámico. El desarrollo del modelo dinámico del pararrotor en el túnel de viento supone asumir una serie de simplificaciones acordes a la ejecución del ensayo. Por ejemplo, dado que el único grado de libertad que presenta el modelo es según el eje $z_{A}$, se debe considerar que la velocidad angular solamente tiene componente en esta dirección, siendo :

$$
\left.\boldsymbol{\omega}\right|_{A}=[0,0, \Omega(t)]^{\prime}
$$

También se considera un modelo de coeficiente de sustentación que varía linealmente con el ángulo de ataque, y un coeficiente de resistencia constante, es decir:

$$
\begin{gathered}
C_{D_{b}}=C_{D} \\
C_{L_{b}}(\alpha)=C_{L_{\alpha}} \alpha
\end{gathered}
$$

El esquema de acciones transmitidas por el pararrotor al punto de anclaje al sistema de montaje (punto O) se observa en la siguiente figura: 


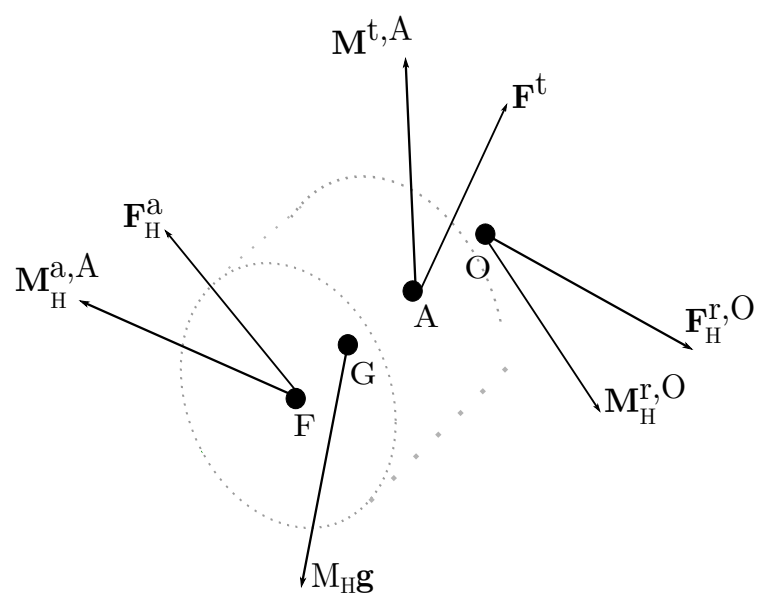

Figura 17: Esquema de transmisión de fuerzas y momentos del pararrotor al sistema de montaje

El punto de acoplamiento O (ver figura 17) se encuentra fijo, por lo que las ecuaciones de Newton referidas a dicho punto resultan:

$$
\mathbf{F}^{t}+\mathbf{F}_{H}^{\nearrow^{\simeq} \simeq 0}+M_{H} \mathbf{g}+\mathbf{F}_{H}^{r, O}=M_{H} \frac{d \mathbf{V}^{\nearrow^{0}}}{d t}=0
$$

donde $\mathbf{F}^{t}$ es la fuerza que transmiten las palas, $M_{H} \mathbf{g}$ el peso del cilindro y $\mathbf{F}_{H}^{r, O}$ la fuerza de reacción que recibe el pararrotor a través del punto O.

La ecuación de Euler queda como sigue:

$\left(\mathbf{M}_{H}^{a, F^{\star}} \simeq 0\right.$ OF $\left.\wedge \mathbf{F}_{H}^{\sigma^{\prime}} \simeq 0\right)+\left(\mathbf{M}^{t, A}+\mathbf{O A} \wedge \mathbf{F}^{t}\right)+\mathbf{O G} \wedge M_{H} \mathbf{g}+\mathbf{M}_{H}^{r, O}=\frac{d \mathbf{h}_{H}}{d t}=I_{3} \frac{d \Omega}{d t} \mathbf{k}_{A}$

donde el término $\left(\mathbf{M}^{t, A}+\mathbf{O A} \wedge \mathbf{F}^{t}\right)$ corresponde al momento que transmiten ambas palas al punto A, OG $\wedge M_{H} \mathbf{g}$ es el momento del peso del cilindro respecto al punto de acoplamiento $\mathrm{O}$ y $\mathbf{M}_{H}^{r, O}$ es el momento de reacción sobre el pararrotor en el punto de acoplamiento $\mathrm{O}$. La derivada temporal del momento cinético del rotor se expresa en su componente $\mathbf{k}_{A}$, ya que en las otras dos direcciones dicha derivada es nula. La fuerza y el momento que el pararrotor transmite en el punto $\mathrm{O}$ viene dado por las siguientes expresiones:

$$
\begin{gathered}
\mathbf{F}_{H}^{r, O}=-\mathbf{F}^{t}-M_{H} \mathbf{g} \\
\mathbf{M}_{H}^{r, O}=-\left(\mathbf{M}^{t, A}+\mathbf{O A} \wedge \mathbf{F}^{t}\right)-\left(\mathbf{O G} \wedge M_{H} \mathbf{g}\right)-I_{3} \frac{d \Omega}{d t} \mathbf{k}_{A}
\end{gathered}
$$


La proyección de estas ecuaciones sobre el eje $z_{A}$ da lugar a la ecuación que describe la dinámica del modelo en el túnel de viento:

$$
\mathbf{M}^{t, A} \cdot \mathbf{k}_{A}+\underbrace{\left(\mathbf{O A} \wedge \mathbf{F}^{t}\right) \cdot \mathbf{k}_{A}}_{0}+\underbrace{\left(\mathbf{O G} \wedge M_{H} \mathbf{g}\right) \cdot \mathbf{k}_{A}}_{0}=I_{3} \frac{d \Omega}{d t} \mathbf{k}_{A}
$$

Como resultado del desarrollo de la ecuación de momentos, tanto aerodinámicos como inerciales, resulta la siguiente ecuación diferencial ordinaria no lineal:

$$
M_{z A}^{t, A}-I_{3} \frac{d \Omega(t)}{d t}=0
$$

Esta ecuación representa el modelo del pararrotor en el túnel de viento. Las expresiones que representan a cada uno de los términos que la componen son algebráicamente extensas, por lo que resulta conveniente explorar técnicas que permitan su interpretación cualitativa antes de proceder a su resolución.

El término $M_{z A}^{t, A}$ de la ecuación (70) puede expresarse en forma funcional del modo siguiente:

$$
M_{z A}^{t, A}=m\left(\Omega, \psi, \theta_{0}, \theta_{1 S}, \theta_{1 C}\right) \frac{d \Omega}{d t}-f\left(\Omega, \psi, \theta_{0}, \theta_{1 S}, \theta_{1 C}\right)
$$

donde $m$ y $f$ son funciones de la velocidad angular $\Omega(t)$, del ángulo de azimut $\psi(t)$ y de los ángulos de paso cíclicos y colectivos, $\theta_{0}, \theta_{1 S}$ y $\theta_{1 C}$. La ecuación diferencial (70)se resuelve en conjunto con la ecuación siguiente:

$$
\frac{d \psi}{d t}=\Omega
$$

Ambas ecuaciones (70 y 72) dan lugar a la formulación canónica del problema:

$$
\left[\begin{array}{cc}
I_{3}+m & 0 \\
0 & 1
\end{array}\right]\left[\begin{array}{l}
\frac{d \Omega}{d t} \\
\frac{d \psi}{d t}
\end{array}\right]=\left[\begin{array}{l}
f \\
\Omega
\end{array}\right]
$$

El sistema (73) es un subsistema de (33), concretamente es la extracción de los elementos en común de las filas y columnas 6 y 9 . Si se indican los elementos de la matriz de masa en el sistema (33) como $a_{i, j}$ resulta que:

$$
\begin{aligned}
I_{3}+m & =a_{6,6}, 0=a_{6,9} \\
0 & =a_{9,6}, 1=a_{9,9}
\end{aligned}
$$


El resto de las variables de estado se establecen constantes en el problema simplificado, como se indica a continuación:

$$
\left[\begin{array}{c}
u \\
v \\
w \\
\omega_{x} \\
\omega_{y} \\
\Theta \\
\Phi \\
r_{G_{1}} \\
r_{G_{2}} \\
r_{G_{3}}
\end{array}\right]=\left[\begin{array}{c}
\mathbf{0}_{5 \times 1} \\
\frac{\pi}{2} \\
\mathbf{0}_{4 \times 1}
\end{array}\right]
$$

A fin de cuantificar el orden de magnitud con el que contribuye cada uno de los términos que modelan las fuerzas y momentos que actúan sobre el pararrotor, como así también obtener una idea general del comportamiento de la ecuación diferencial con la que se modelan las variables cinemáticas del pararrotor, se realiza un análisis en series de Taylor multivariable para cada una de las componentes de fuerzas y momentos, tanto inerciales como aerodinámicas.

\subsubsection{Desarrollo en serie de Taylor del modelo en túnel de viento}

El subsistema (73) modela el comportamiento del pararrotor en el túnel de viento. El análisis que se realiza en este apartado está orientado a determinar de modo cuantitativo la influencia de las variables de estado y de control en el desempeño del pararrotor. Para esto, se realiza un análisis en serie de Taylor multivariable reteniendo distintos órdenes de magnitud a fin de evaluar el impacto del truncamiento en la solución del sistema. La simplificación del sistema en un intervalo de tiempo relativamente pequeño proporciona información valiosa sobre como modifican cada una de las variables el comportamiento del sistema, ya que la relación entre dichas variables se visualiza de manera más clara en expresiones de orden reducido.

En términos generales, el desarrollo en serie de Taylor de segundo orden implica redefinir la función $f$ como sigue

$$
f(\mathbf{x}) \cong f(\mathbf{a})+\nabla f(\mathbf{a}) \cdot(\mathbf{x}-\mathbf{a})+(\mathbf{x}-\mathbf{a})^{\prime} \cdot H f(\mathbf{a})(\mathbf{x}-\mathbf{a})
$$

Donde $\mathrm{H}$ indica el operador Hessiano $\frac{\partial^{2} f}{\partial x_{i} \partial x_{j}}$ y a es una configuración de equilibrio del vector de estado. 


\subsubsection{Simplificación de términos inerciales}

Las expresiones que gobiernan el comportamiento de los momentos inerciales presentan términos extremadamente complejos para analizarlas en su totalidad. Sin embargo, se pueden estudiar los rasgos generales de dichos elementos asumiendo ciertas hipótesis simplificativas.

Una hipótesis de trabajo que se utilizará es la que se conoce como hipótesis de lámina, en la que se considera que los momentos de inercia de la pala cumplen la relación $I_{z_{B}}=I_{x_{B}}+I_{y_{B}}$. Esto implica considerar a la pala, desde un punto de vista másico como una placa de dimensiones $l_{x_{B}}$, $l_{y_{B}}$ y $l_{z_{B}}$, con $l_{z_{B}} \cong l_{y_{B}} \ll l_{x_{B}}$. Bajo esta hipótesis, el tensor (3.8) resulta:

$$
\mathbf{I}_{B} \approx\left[\begin{array}{ccc}
I_{x_{B}} & 0 & 0 \\
0 & I_{y_{B}} & 0 \\
0 & 0 & I_{x_{B}}+I_{y_{B}}
\end{array}\right]
$$

Otro aspecto que contribuye a la simplificación es el análisis de expresiones de menor orden. Por ejemplo, reteniendo términos de primer orden para los parámetros de control en los momentos inerciales transmitidos por las palas al cilindro (ecuación (50) ) y considerando la hipótesis de lámina, se tienen la siguientes expresiones para los momentos:

$$
\begin{gathered}
M_{x_{A}}^{i, A}=-2 I_{x_{B}} \theta_{1 S} \frac{d \Omega}{d t} \\
M_{y_{A}}^{i, A}=2 I_{x_{B}} \theta_{1 C} \frac{d \Omega}{d t} \\
M_{z_{A}}^{i, A}=\frac{d \Omega(t)}{d t}\left[-2 M_{b} e^{2}-4 M_{b} e x_{G B}-2\left(I_{y_{B}}+I_{x_{B}}\right)\right]
\end{gathered}
$$

En estas expresiones se observa la dependencia directa del momento inercial con las variaciones temporales de la velocidad angular.

Además, para las fuerzas de inercia se tiene que son nulas en el desarrollo en serie de Taylor con términos de primer orden:

$$
\mathbf{F}_{b}^{i}=0
$$

Ahora bien, si se retienen términos de segundo orden para los parámetros de control (esto es, se retienen los términos en los que aparecen productos entre los 
distintos parámetros del vector de control) se tiene que las expresiones de momentos según los ejes $x_{A}$ e $y_{A}$ no se modifican, pero sí lo hace la expresión del momento inercial según la dirección $z_{A}$. A continuación se muestra esta expresión, en la que se identifican los términos cuadráticos de los parámetros de control:

$$
\begin{aligned}
M_{z_{A}}^{i, A}= & {\left[2 I_{x_{B}} \cos ^{2} \psi\left(\theta_{1 C}^{2}-\theta_{1 S}^{2}\right)+4 I_{x_{B}} \cos \psi \sin \psi \theta_{1 C} \theta_{1 S}+2 I_{x_{B}}\left(\theta_{0}^{2}+\theta_{1 S}^{2}\right)-\right.} \\
& \left.-2 M_{b} e^{2}-4 M_{b} e x_{G B}-2\left(I_{y_{B}}+I_{x_{B}}\right)\right] \frac{d \Omega(t)}{d t}+ \\
& +4\left[\left(2 \cos ^{2} \psi-1\right) \theta_{1 C} \theta_{1 S}-\cos \psi \sin \psi\left(\theta_{1 S}^{2}-\theta_{1 C}^{2}\right)\right] I_{x_{B}} \Omega^{2}(t)
\end{aligned}
$$

Las fuerzas inerciales, reteniendo términos de segundo orden para los parámetros de control, también son nulas.

\subsubsection{Simplificación de términos aerodinámicos}

Del mismo modo que en el caso de los momentos inerciales, se busca expresar el momento aerodinámico, bajo algunas consideraciones y definiciones, de manera sencilla dando cuenta de los principales parámetros que inciden.

Respecto a las acciones de control, definidas por los ángulos $\theta_{0}, \theta_{1 S}$ y $\theta_{1 C}$, se retienen términos de hasta primer orden. Esto significa que los términos que aparecen en las expresiones son, a lo sumo, lineales con $\theta_{0}, \theta_{1 C}$ o $\theta_{1 S}$.

Para la excentricidad $e$ y la coordenada $y_{P_{b}}$ del centro de presiones se retienen términos de orden 0 , es decir que se suponen nulas.

Si además se definen los coeficientes adimensionales $\mu_{z A}=U_{0}\left(\Omega R_{b}\right)^{-1}$ y $X_{P}=x_{P_{b}} R_{b}^{-1}$, donde $U_{0}$ es la velocidad de referencia de la corriente de aire, $\Omega$ la velocidad angular de referencia, $R_{b}$ el radio de adimensionalización y $x_{P_{b}}$ la coordenada según $x_{B_{b}}$ de la posición del centro de presiones de la pala, se puede afirmar que estas relaciones son pequeñas. Por lo tanto, también se retienen términos de hasta primer orden. Con este cambio de variables y el desarrollo en serie de Taylor resultan las siguientes expresiones para el momento aerodinámico según el sistema de ejes A:

$$
\begin{gathered}
M_{x_{A}}^{a, A}=\rho S \Omega(t)^{2} X_{P}^{3} \sin \psi C_{L \alpha}\left(\theta_{1 C} \cos \psi+\theta_{1 S} \sin \psi\right) \\
M_{y_{A}}^{a, A}=-\rho S \Omega(t)^{2} X_{P}^{3} \cos \psi C_{L \alpha}\left(\theta_{1 C} \cos \psi+\theta_{1 S} \sin \psi\right)
\end{gathered}
$$




$$
M_{z_{A}}^{a, A}=-\rho S \Omega(t)^{2} X_{P}^{3}\left(C_{D}+C_{L \alpha} \theta_{0} \mu_{z A}\right)
$$

Mientras que las fuerzas aerodinámicas resultan:

$$
\begin{gathered}
F_{x_{A}}^{a}=\rho S \Omega(t)^{2} X_{P} R_{b}^{2} C_{L \alpha} \mu_{z_{A}} \sin \psi\left(\theta_{1 C} \cos \psi+\theta_{1 S} \sin \psi\right) \\
F_{y_{A}}^{a}=-\rho S \Omega(t)^{2} X_{P} R_{b}^{2} \mu_{z_{A}} \cos \psi C_{L \alpha}\left(\theta_{1 C} \cos \psi+\theta_{1 S} \sin \psi\right) \\
F_{z_{A}}^{a}=\rho S \Omega(t)^{2} X_{P} R_{b}^{2}\left[X_{P} C_{L \alpha} \theta_{0}-\mu_{z_{A}}\left(C_{D}+C_{L \alpha}\right)\right]
\end{gathered}
$$

Se obtienen expresiones más complejas si se retienen términos de hasta segundo orden para $\mu_{z A}$. Para los parámetros de control $\left(\theta_{0}, \theta_{1 S}\right.$ y $\left.\theta_{1 C}\right)$ se retienen hasta los términos de primer orden y para $y_{C P}$ y $e$ de orden cero:

$$
\begin{gathered}
M_{x_{A}}^{a, A}=\rho S \Omega(t)^{2} X_{P} R_{b}^{3} \sin \psi C_{L \alpha}\left[\theta_{1 S} \sin \psi\left(X_{P}^{2}+\frac{\mu_{z_{A}}}{2}\right)+\theta_{1 C} \cos \psi\left(X_{P}^{2}+\frac{\mu_{z_{A}}}{2}\right)\right] \\
M_{y_{A}}^{a, A}=-\rho S \Omega(t)^{2} X_{P} R_{b}^{3} \cos \psi C_{L \alpha}\left[\theta_{1 S} \sin \psi\left(X_{P}^{2}+\frac{\mu_{z_{A}}}{2}\right)+\theta_{1 C} \cos \psi\left(X_{P}^{2}+\frac{\mu_{z_{A}}}{2}\right)\right] \\
M_{z_{A}}^{a, A}=-\rho S \Omega(t)^{2} X_{P} R_{b}^{2}\left[C_{D}+C_{L \alpha} \mu_{z_{A}} \theta_{0}+\left(\frac{C_{D}}{2}-C_{L \alpha} \mu_{z A}^{2}\right)\right]
\end{gathered}
$$

Las fuerzas aerodinámicas que generan las palas no modifican su expresión al aumentar el orden del factor $\mu_{z_{A}}$.

Con las expresiones anteriores se puede formular nuevamente la ecuación (70), reemplazando las expresiones para los momentos. 
Tanto en (81) como en (83) se observa la dependencia del momento aerodinámico del cuadrado de la velocidad angular $\left(\Omega(t)^{2}\right)$, mientras que la variación temporal de la velocidad angular $\left(\frac{d \Omega(t)}{d t}\right)$ no interviene. También se puede observar que el control del pararrotor en los planos $y_{A}-z_{A}$, dominado por el momento $M_{x_{A}}^{a, A}$, y $x_{A}-z_{A}$, dominado por el momento $M_{y_{A}}^{a, A}$, es realizado por los parámetros $\theta_{1 S} \mathrm{y}$ $\theta_{1 C}$. El movimiento en el plano $x_{A}-y_{A}$ está dominado por el parámetro $\theta_{0}$, que es el único parámetro controlable en la ecuación $M_{z_{A}}^{a, A}$ del sistema (83).

Por último, si se analizan las expresiones que se obtienen retienendo términos de segundo orden tanto para $\mu_{z_{A}}$ como para $\theta_{0}, \theta_{1 S}$ y $\theta_{1 C}$ se puede observar que son idénticas a las anteriores (ecuación (81)), en el caso de que el orden para los parámetros de control era de primer grado. Esto da un indicio de que los momentos aerodinámicos pueden ser considerados de orden uno al plantear la ecuación de conservación del momento angular.

\subsubsection{Solución del modelo en series de Taylor del pararrotor en túnel de viento}

Resumiendo, el momento estimado al variar cíclicamente el paso de las palas se compone de dos términos, uno inercial y otro aerodinámico. La expresión analítica para el momento total se puede obtener de la suma de las componentes inerciales y aerodinámicas, obteniéndose un sistema de ecuaciones diferenciales ordinarias no lineales de segundo orden:

$$
\begin{gathered}
M_{z A}^{a, A}(\psi(t), t)+M_{z A}^{i, A}(\psi(t), t)-I_{3} \frac{d \Omega(t)}{d t}=0 \\
\frac{d \psi}{d t}=\Omega(t)
\end{gathered}
$$

La solución de este sistema de ecuaciones entrega como resultado el perfil de variación temporal del momento y de la velocidad angular del pararrotor en el túnel de viento.

De la evaluación de los términos que corresponden a cada uno de los momentos resulta la ecuación diferencial ordinaria no lineal que modela el comportamiento de la velocidad angular del pararrotor. Como se muestra en el apartado correspondiente, los términos inerciales y aerodinámicos pueden ser representados por desarrollos en serie de Taylor para retener los términos de primer orden para facilitar la solución numérica del sistema (70).

Con estas hipótesis de simplificación y suponiendo excentricidad nula $(e=0)$, la ecuación a resolver para el modelo del pararrotor en túnel de viento resulta: 


$$
\begin{aligned}
& {\left[-2\left(I_{y_{B}}-I_{z_{B}}\right)\left(1-\cos \psi^{2}\right)-2\left(I_{y_{B}}-I_{z_{B}}\right) \theta_{0}^{2}-2 I_{z_{B}}-I_{H}\right] \frac{d \Omega(t)}{d t}} \\
& -\left[4\left(I_{y_{B}}-I_{z_{B}}\right) \cos \psi \sin \psi \theta_{1 S}^{2}+x_{c p}^{3} \rho S C_{D}\right] \Omega(t)^{2} \\
& -x_{c p}^{3} \rho S C_{D} \frac{U_{0}}{R_{b}} C_{L \alpha} \theta_{0} \Omega(t)+x_{c p}^{3} \rho S \frac{U_{0}^{2}}{R_{b}^{2}}\left(C_{L \alpha}-\frac{C_{D}}{2}\right)=0
\end{aligned}
$$

expresión que resulta ser una ecuación diferencial cuadrática, del tipo:

$$
\left.\frac{d \Omega(t)}{d t}+A(\psi)\right) \Omega(t)^{2}+B(\psi) \Omega(t)+C(\psi)=0
$$

en donde:

$$
\begin{aligned}
& A(\psi)=\frac{4\left(I_{y_{B}}-I_{z_{B}}\right) \cos \psi \sin \psi \theta_{1 S}^{2}+x_{c p}^{3} \rho S C_{D}}{2\left(I_{y_{B}}+I_{z_{B}}\right)\left(1-\cos \psi^{2}\right)+2\left(I_{y_{B}}+I_{z_{B}}\right) \theta_{0}^{2}+2 I_{z_{B}}+I_{3}} \\
& B(\psi)=\frac{4\left(I_{y_{B}}-I_{z_{B}}\right) \cos \psi \sin \psi \theta_{1 S}^{2}-x_{c p}^{3} \rho S C_{D}}{2\left(I_{y_{B}}+I_{z_{B}}\right)\left(1-\cos \psi^{2}\right)+2\left(I_{y_{B}}+I_{z_{B}}\right) \theta_{0}^{2}+2 I_{z_{B}}+I_{3}} \\
& C(\psi)=\frac{x_{c p}^{3} \rho S U_{0}\left(\frac{1}{2} C_{D}+C_{L \alpha}\right)}{2\left(I_{y_{B}}+I_{z_{B}}\right)\left(1-\cos \psi^{2}\right)+2\left(I_{y_{B}}+I_{z_{B}}\right) \theta_{0}^{2}+2 I_{z_{B}}+I_{3}}
\end{aligned}
$$

La expresión (85) no presenta a la vista de forma clara elementos para el análisis cualitativo del comportamiento del dispositivo, por lo que, mediante la definición de una serie de parámetros adimensionales, se adimensionaliza la ecuación:

$$
\begin{gathered}
d=\frac{I_{z_{B}}-I_{y_{B}}}{I_{z_{B}}} \\
h=\frac{I_{3}}{I_{z_{B}}} \\
X_{P}=\frac{x_{P_{b}}}{R_{b}} \\
M=\frac{\rho R_{b}^{3} S}{I_{z_{B}}} \\
\omega(\psi)=\frac{\Omega(t) R_{b}}{U_{0}}
\end{gathered}
$$


y reemplazando en la ecuación (85) se puede reescribirla como sigue:

$$
\begin{aligned}
\left\{d\left(1-\cos \psi^{2}\right) \theta_{1 S}^{2}+d \theta_{0}^{2}-1-\frac{h}{2}\right\} \omega(\psi) & \frac{d \omega(\psi)}{d \psi}+\left\{2 d \cos \psi \sin \psi \theta_{1 S}^{2}-X_{c p}^{3} M \frac{C_{D}}{2}\right\} \omega(\psi)^{2}- \\
& -\frac{1}{2} X_{c p}^{2} M C_{L \alpha} \theta_{0} \omega(\psi)+\frac{1}{2} X_{c p} M\left(C_{L \alpha}-\frac{C_{D}}{2}\right)=0
\end{aligned}
$$

Se puede identificar en la ecuación anterior una estructura del tipo:

$$
\omega(\psi) \frac{d \omega(\psi)}{d \psi}+A(\psi) \omega(\psi)+B(\psi) \omega(\psi)^{2}+C(\psi)=0
$$

que resulta una ecuación diferencial de Abel de segundo tipo clase A para la cual no existe solución analítica general conocida [31].

Para obtener la solución se formula el problema desarrollando una función de MATLAB ß, y se utiliza el solver ode45.

Existen distintos métodos para obtener la solución del sistema. La familia de métodos elegida para aproximar la solución corresponde a los métodos de Runge-Kutta, entre los que se pueden citar el método Bogacki-Shampine (de segundo y tercer orden) o Dormand-Prince (de cuarto y quinto orden). Según el método seleccionado serán la cantidad de coeficientes necesarios para la realización de la integración numérica. Los parámetros generales del sistema se presentan en el siguiente cuadro, en los que se fija una configuración del vector de control determinando los valores de $\theta_{0}, \theta_{1 C}$ y $\theta_{1 S}$.

\begin{tabular}{cc}
\hline \hline Parámetro & Valor \\
\hline $\mathrm{C}_{D}$ & 0,3 \\
$\mathrm{C}_{L \alpha}$ & $1,441 / \mathrm{rad}$ \\
$\theta_{0}$ & {$[0,0690,1040,169] \mathrm{rad}$} \\
$\theta_{1 S}$ & $0 \mathrm{rad}$ \\
$\theta_{1 C}$ & $0 \mathrm{rad}$ \\
$\mathrm{u}_{1 T}$ & $-5 \mathrm{~m} / \mathrm{s}$ \\
$\mathrm{I}_{3}$ & $19,15 \cdot 10^{-4} \mathrm{kgm}^{2}$ \\
$\mathrm{I}_{z_{B}}$ & $3,64 \cdot 10^{-4} \mathrm{kgm}^{2}$ \\
$\mathrm{I}_{y_{B}}$ & $3,25 \cdot 10^{-4} \mathrm{kgm}^{2}$ \\
$\omega_{0 i}$ & $2[-]$ \\
\hline
\end{tabular}

Cuadro 1: Parámetros generales para la resolución del sistema (84) 
Para las condiciones detalladas en el cuadro (1) se grafica en la figura (18) la solución del sistema de ecuaciones diferenciales ordinarias (84).

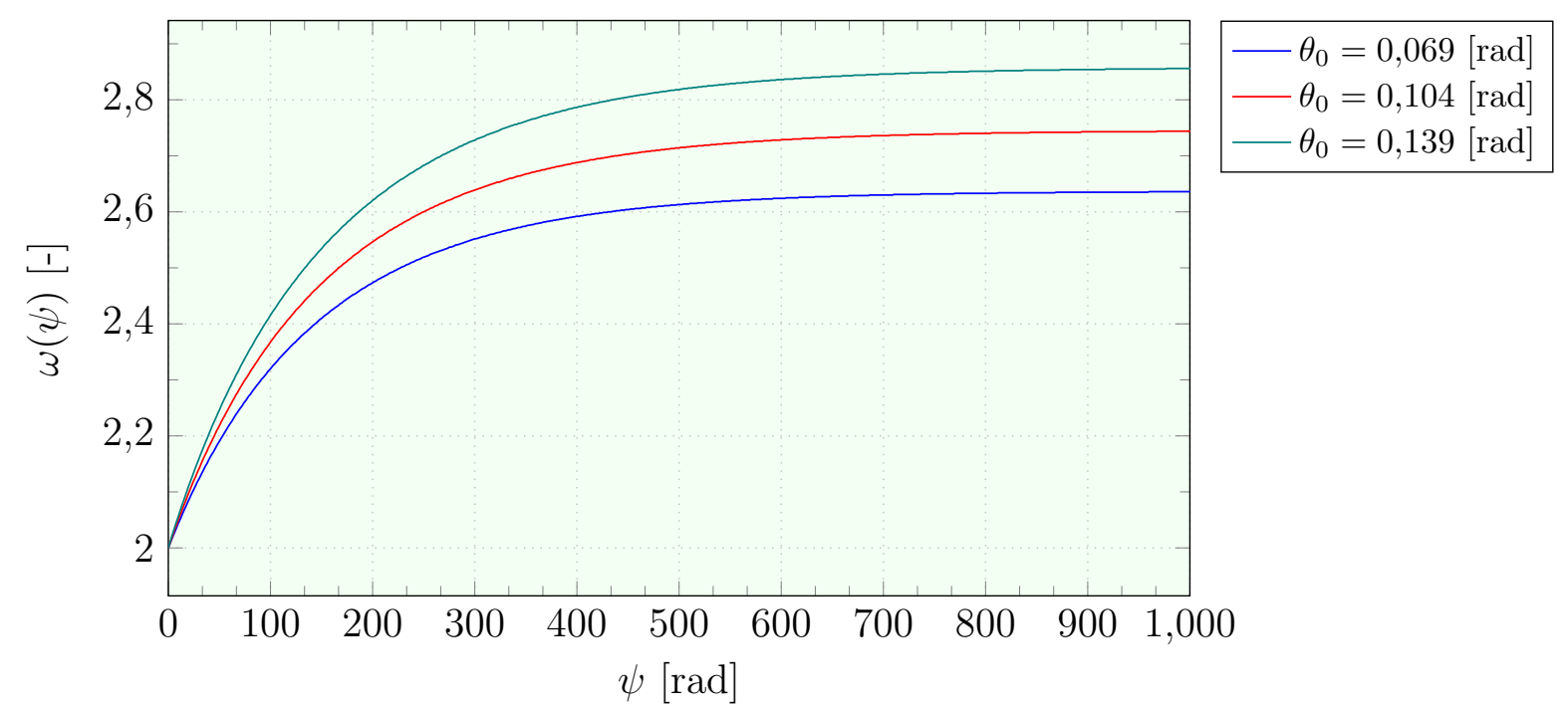

Figura 18: Solución para la ecuación adimensionalizada de la velocidad angular en el túnel de viento con paso cíclico nulo $\left(\theta_{1 S}=\theta_{1 C}=0\right)$.

También se muestra el comportamiento de las variables para el caso en el que se introduce un paso cíclico distinto de cero. Los casos analizados son los que se caracterizan en el siguiente cuadro:

\begin{tabular}{cc}
\hline \hline Parámetro & Valor \\
\hline $\mathrm{C}_{D}$ & 0,3 \\
$\mathrm{C}_{L \alpha}$ & $1,441 / \mathrm{rad}$ \\
$\theta_{0}$ & $0,104 \mathrm{rad}$ \\
$\theta_{1 S}$ & {$[0,0690,1040,169] \mathrm{rad}$} \\
$\theta_{1 C}$ & $0 \mathrm{rad}$ \\
$\mathrm{u}_{1 T}$ & $-5 \mathrm{~m} / \mathrm{s}$ \\
$\mathrm{I}_{3}$ & $19,15 \cdot 10^{-4} \mathrm{kgm}^{2}$ \\
$\mathrm{I}_{z_{B}}$ & $3,64 \cdot 10^{-4} \mathrm{kgm}^{2}$ \\
$\mathrm{I}_{y_{B}}$ & $3,25 \cdot 10^{-4} \mathrm{kgm}^{2}$ \\
$\omega_{0 i}$ & $2[-]$ \\
\hline
\end{tabular}

Cuadro 2: Parámetros generales para la resolución del sistema (84) 


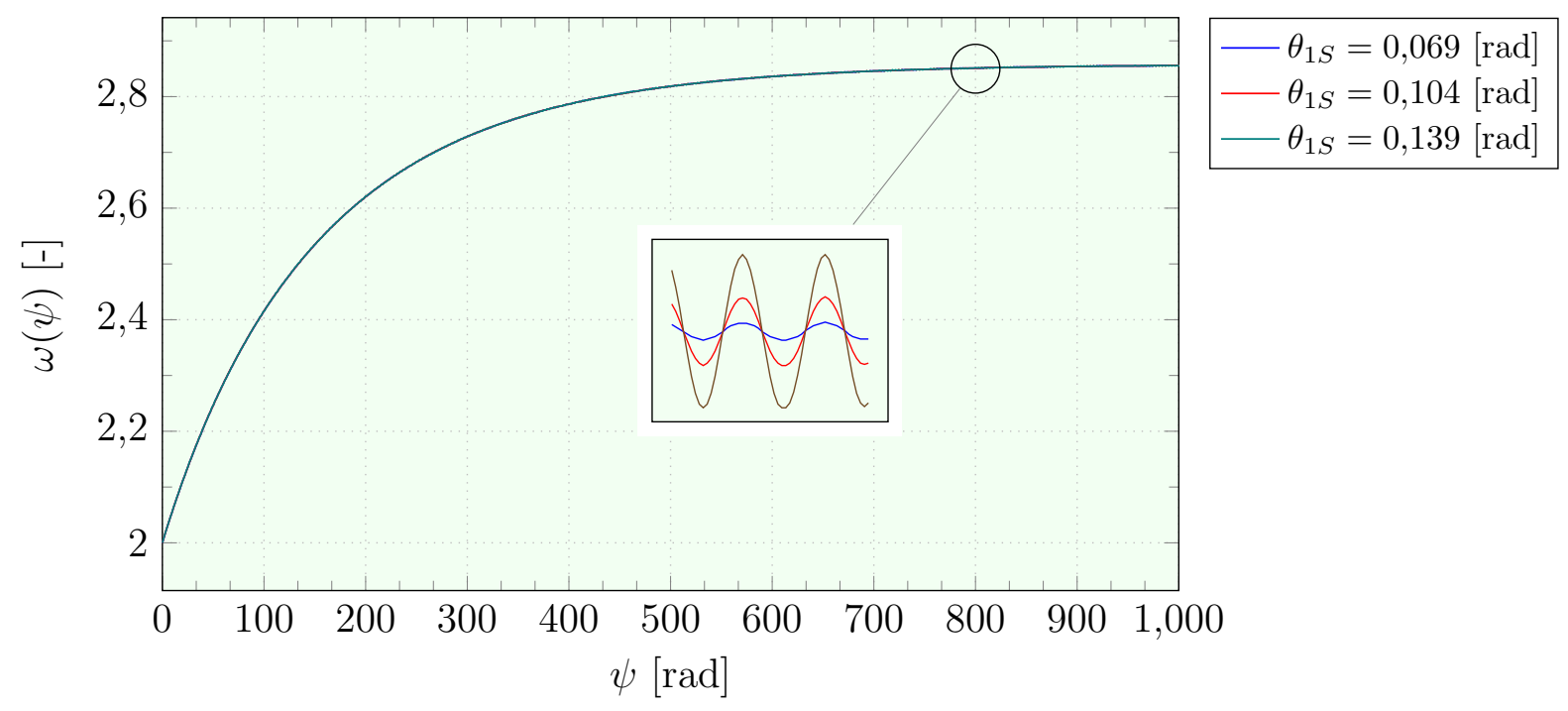

Figura 19: Solución para la ecuación adimensionalizada de la velocidad angular en el túnel de viento con un ángulo de paso colectivo constante $\left(\theta_{0}=0,104 \mathrm{rad}\right)$ y $\theta_{1 S}$ variable. El detalle muestra la oscilación en función del ángulo de paso cíclico.

La recuperación de las variables dimensionales se realiza por medio de la definición de los parámetros adimensionales, siendo $\Omega(t)=\omega(\psi) U_{0}\left(R_{b}\right)^{-1}$. Mediante esta transformación se obtienen las gráficas correspondientes a la velocidad angular $\Omega(t)$ para los casos con ángulo de paso cíclico nulo y variable:

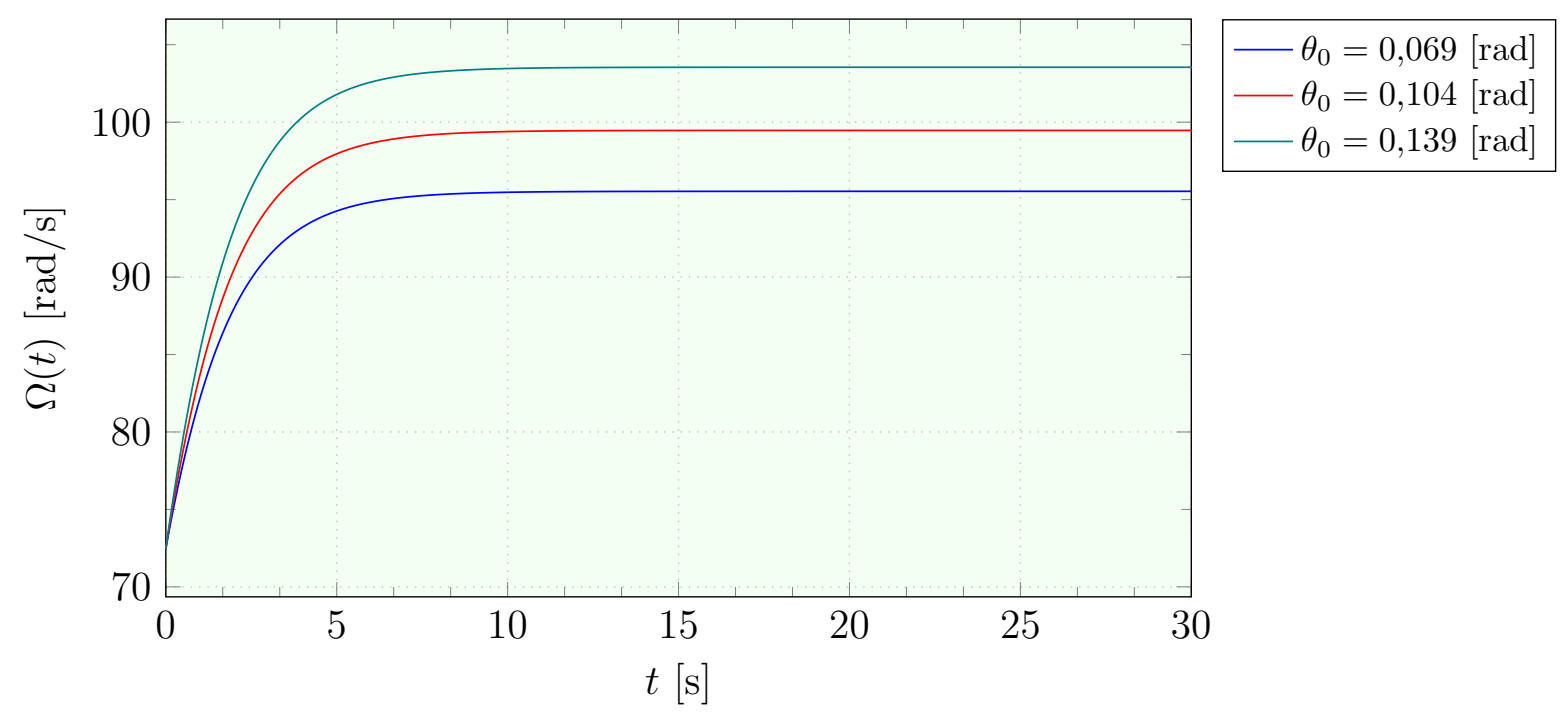

Figura 20: Velocidad angular del modelo teórico para el caso túnel de viento con paso cíclico nulo $\left(\theta_{1 S}=\theta_{1 C}=0\right)$. 


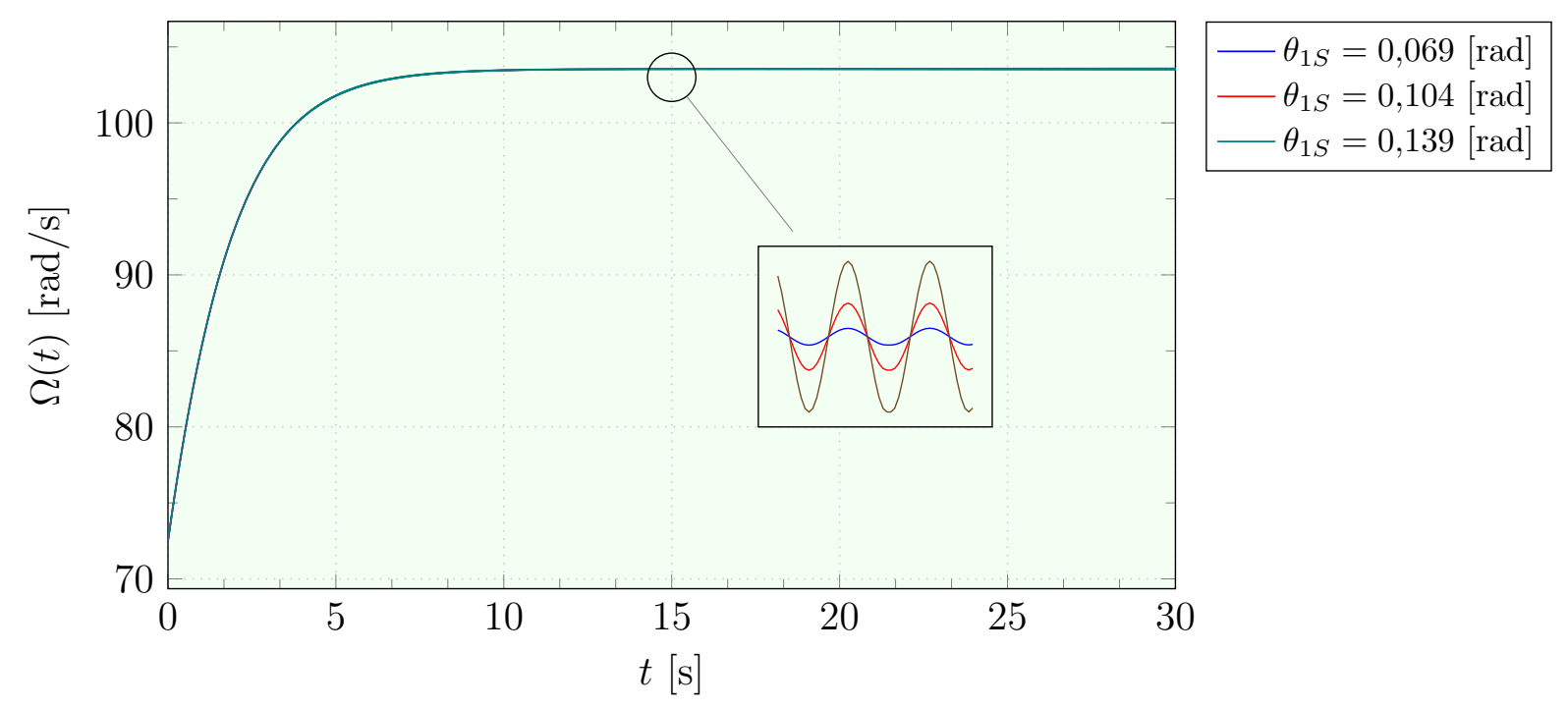

Figura 21: Velocidad angular del pararrotor en el túnel de viento a partir de la velocidad angular adimensional para un ángulo de paso colectivo constante $\left(\theta_{0}=\right.$ $0,104 \mathrm{rad})$ y $\theta_{1 S}$ variable. El detalle muestra la oscilación en función del ángulo de paso cíclico.

Por último, se muestra la comparación de la solución del sistema (70) obtenida para el modelo simplificado de fuerzas y momentos sobre el pararrotor y la obtenida para el modelo completo:

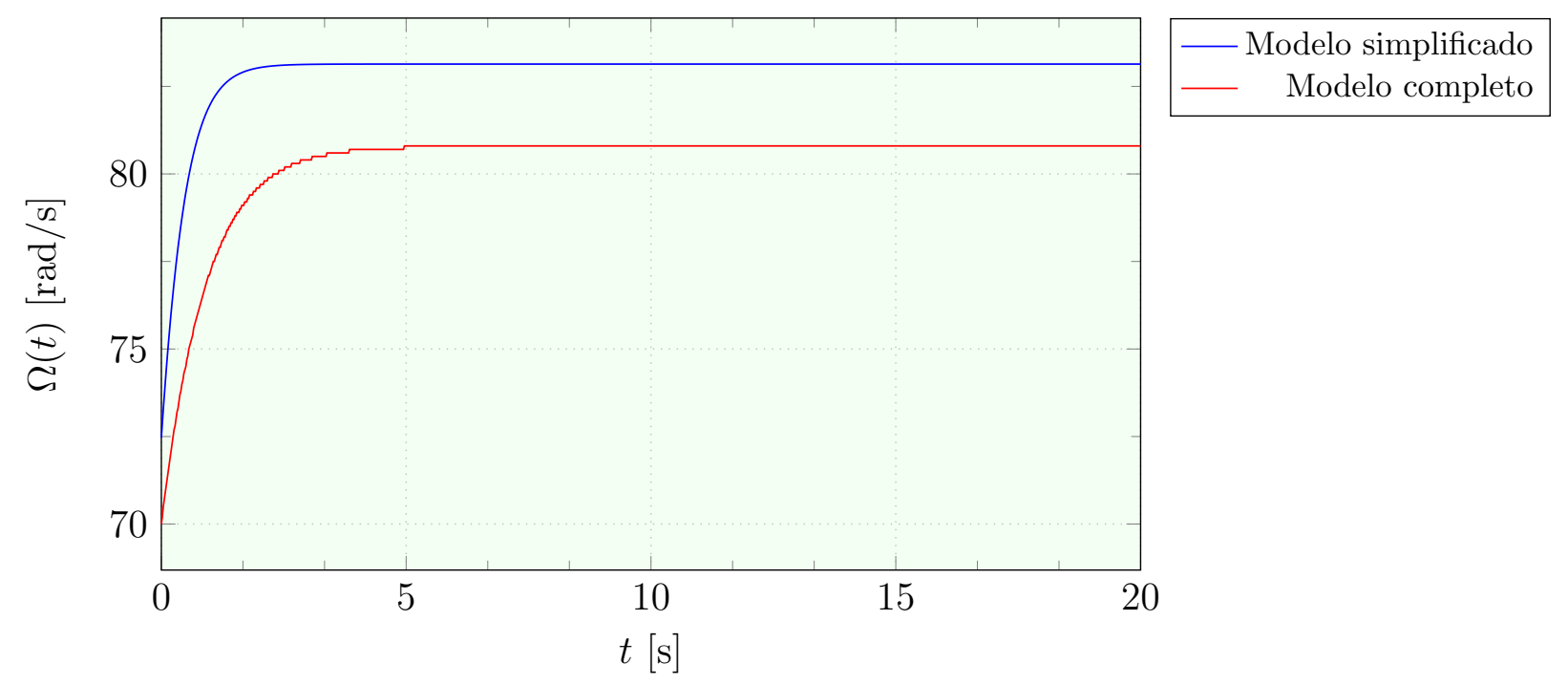

Figura 22: Comparación entre la solución del modelo simplificado y la solución del modelo completo de la velocidad angular en el túnel de viento con paso $\theta_{0}=6^{\circ} \mathrm{y}$ $\left.\theta_{1 S}=0^{\circ}\right)$. 
A partir de la figura 22 se observa que la diferencia según el modelo utilizado para la resolución del sistema (83) alcanza un $4 \%$. Vale decir que el modelo descriptivo desarrollado a partir de las hipótesis simplificativas y el desarrollo en series de Taylor permite el análisis del comportamiento dinámico del pararrotor.

El sistema fue resuelto mediante distintos métodos numéricos, a fin de evaluar la convergencia del mismo. Se utilizaron los métodos de Adam-Bashforth, Bogacki-Shampine y Dormand-Prince, y se observó que la evolución temporal de la diferencia relativa entre iteraciones alcanza el $1 \%$ en un tiempo de cómputo menor a 10 segundos.

\subsubsection{Otros resultados derivados de la resolución del modelo teórico}

La obtención de una solución para la velocidad angular en el túnel de viento permite recuperar parámetros del estado dinámico del pararrotor, como ser fuerzas y momentos, y paramétros aerodinámicos, como por ejemplo los ángulos de ataque de cada una de las palas. Si bien la validación de este modelo se realiza en el capítulo 4, se presentan a continuación, obedeciendo solamente a respetar el orden de desarrollo lógico propuesto en el documento, algunas características de interés que pueden analizarse a partir de este modelo teórico.

Por ejemplo, resulta de interés determinar la incidencia en el momento total de la componente aerodinámica y la inercial. También se pueden estimar parámetros de diseño, como el torque que tienen que aplicar los motores que accionen las palas (momentos respecto al punto $E_{b}$ ) a partir del cálculo de este valor.

\section{- Momentos aerodinámicos e inerciales}

A partir de la resolución del sistema (84) se puede obtener un perfil de la variación del torque aerodinámico, ya sea en sus componenetes en el sistema A1b o A. En la figura 23 se trazan distintas curvas que grafican el valor del momento aerodinámico en función del ángulo de paso cíclico longitudinal $\theta_{1 S}$ para una vuelta del pararrotor, para un ángulo de paso colectivo $\theta_{0}=0,104 \mathrm{rad}$ y un ángulo de paso cíclico lateral $\theta_{1 C}=0$. Este perfil de variación del momento aerodinámico se obtiene utilizando la solución de $\Omega(t)$ obtenida del sistema (70) para la configuración determinada y reemplazando en la expresión desarrollada mediante la herramienta simbólica de cálculo. Nótese que se grafican los momentos aerodinámicos según el eje $x_{A}$. La matriz de transformación (3.3) permite obtener las expresiones de los momentos en el sistema de coordenadas A.

De este modo se obtienen el siguiente conjunto de gráficas presentadas: 


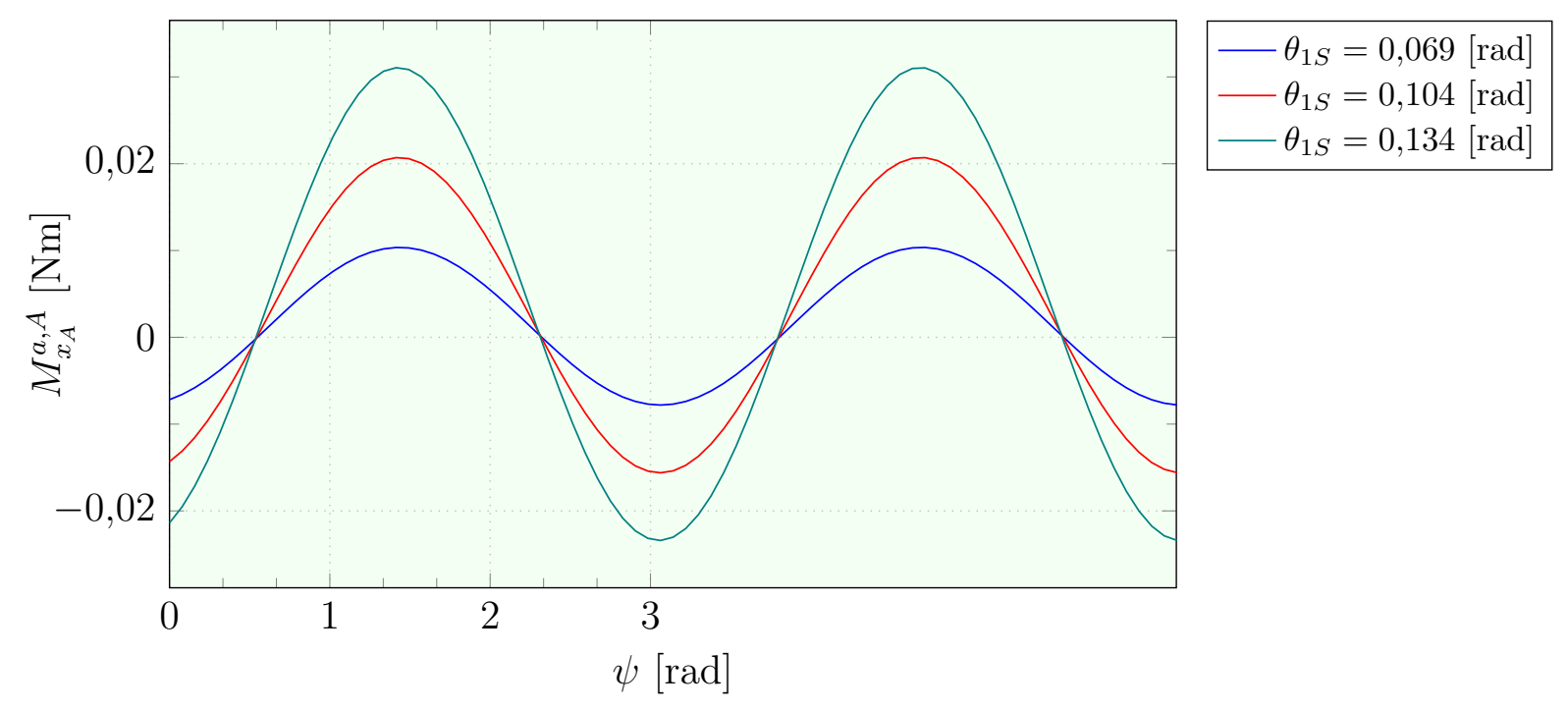

Figura 23: Momento aerodinámico para distintos valores del paso cíclico longitudinal.

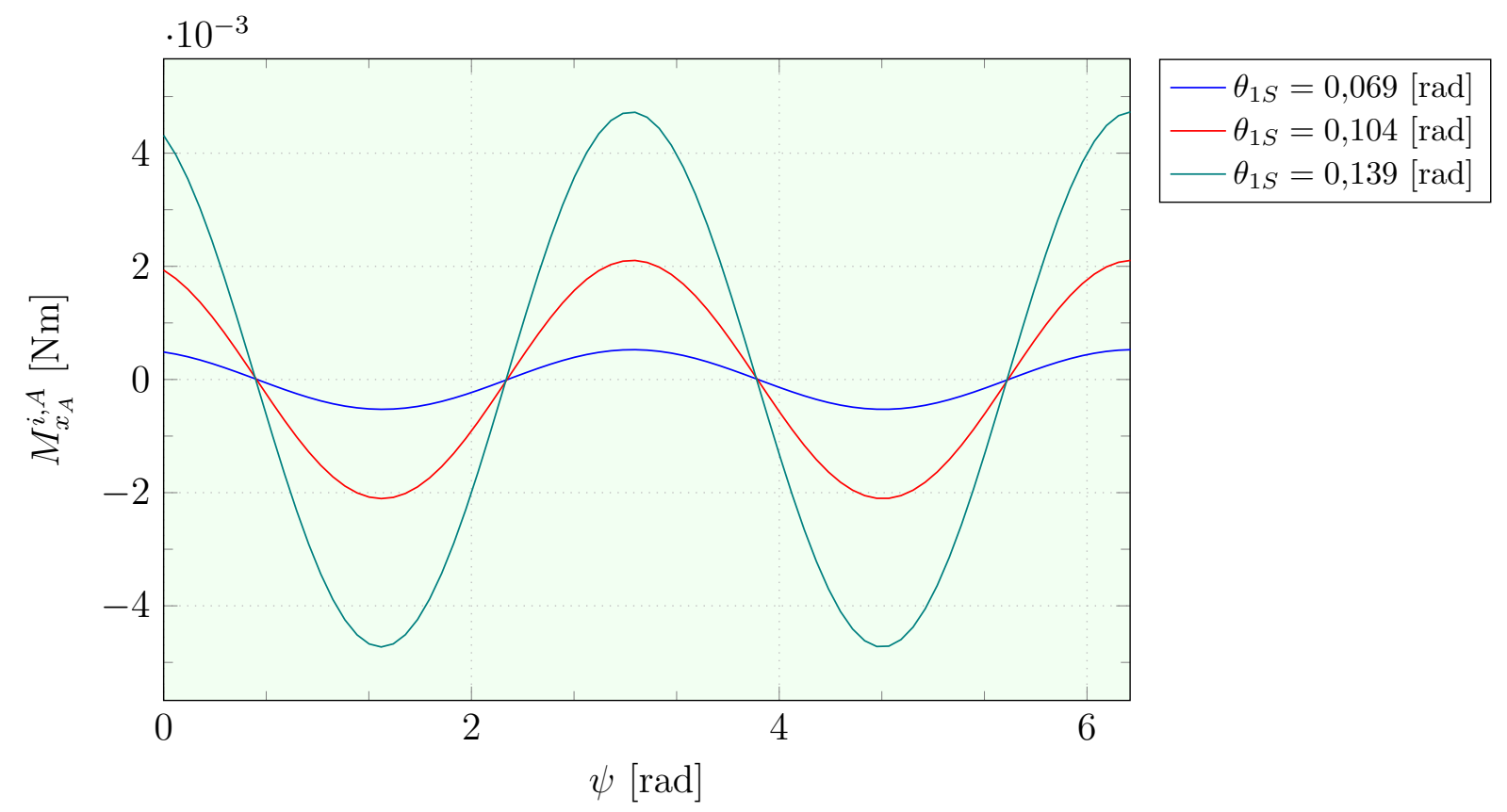

Figura 24: Momento inercial para distintos valores del paso cíclico longitudinal.

Según la figura 23, el momento aerodinámico aumenta su valor máximo conforme lo hace el ángulo $\theta_{1 S}$. Recurriendo a la ecuación (83), puede atribuirse este comportamiento a la relación directa que tiene el momento aerodinámico según $x_{A}$ con el parámetro $\theta_{1 S}$. En esta ecuación se observa que dicho parámetro multiplica una función de $\sin ^{2} \psi$, haciendo las veces de modulador de $M_{x_{A}}^{a, A}$. 
Para el caso de los momentos inerciales, la variación según el paso cíclico puede verse en la figura 24, donde se grafica la solución de la componente inercial de $M_{x_{A}}^{A}$ según el sistema (73) y distintas configuraciones del cíclico longitudinal (el paso cíclico lateral se supone nulo).

Refiriéndose a la ecuación que desarrolla hasta el primer orden el momento inercial (ecuación (78)), se observa que la componente según $x_{A}$ depende en forma lineal del parámetro $\theta_{1 S}$, lo que explica el comportamiento incremental con este parámetro del momento inercial. Además, en el caso de no asignar variaciones cíclicas al ángulo de paso de las palas, el valor de esta componente de momento resulta nulo.

Comparando las magnitudes que alcanzan los momentos en las figuras 23 y 24 se observa que los momentos inerciales son un orden de magnitud menores que los momentos aerodinámicos.

\section{- Momento en el acoplamiento de la pala}

El hecho de conocer el valor que toma el momento que la pala genera en el punto $E_{b}$ tiene una aplicación práctica inmediata en el dimensionado del acoplamiento de la pala y en la selección del tipo de servomecanismo necesario para accionarla.

La figura 25 muestra como evoluciona a lo largo de una vuelta el momento respecto al eje de una pala $\left(M_{x_{B_{b}}}^{a, E_{b}}\right)$. Como es de esperar, el momento aerodinámico respecto al eje $x_{B_{b}}$ aumenta con el ángulo de paso cíclico.

Observando la variación del ángulo de ataque en la vuelta (figura 26), puede verse que el momento en la dirección $x_{B_{b}}$ (figura 25) reproduce el mismo comportamiento. Los valores más pequeños y más altos de $M_{x_{B_{b}}}^{a, E_{b}}$ (en valor absoluto) se corresponden respectivamente con los valores de $\alpha_{b}$. También debe destacarse que el ángulo de ataque que se alcanza es elevado, con lo que se necesita analizar con mayor profundidad el modelo aerodinámico utilizado para establecer los coeficientes aerodinámicos. La utilización de un modelo lineal genera fuerzas aerodinámicas teóricas incluso a elevados valores del ángulo de ataque, sin contemplar la posibilidad de la entrada en pérdida de los perfiles.

La figura 26 muestra la variación del ángulo de ataque $\alpha_{b}$ en función del ángulo de azimut $\psi$, para distintos valores de ángulo de paso cíclico longitudinal.

Este análisis brinda elementos para el dimensionado del eje y de los servomecanismos necesarios para realizar la actuación necesaria. 


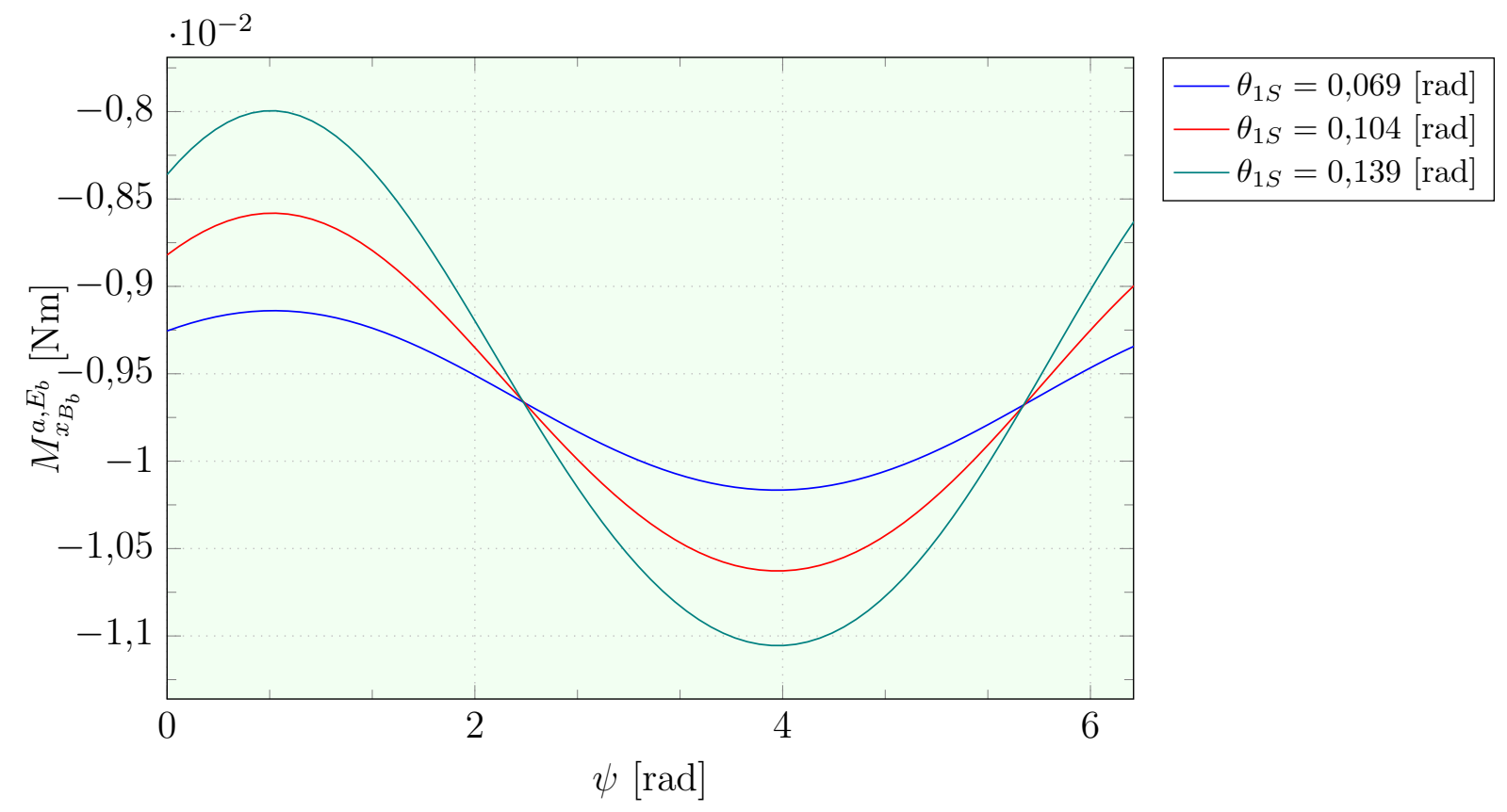

Figura 25: Momento aerodinámico respecto al eje $x_{B}$ de la pala $b$ para distintos valores del paso cíclico longitudinal.

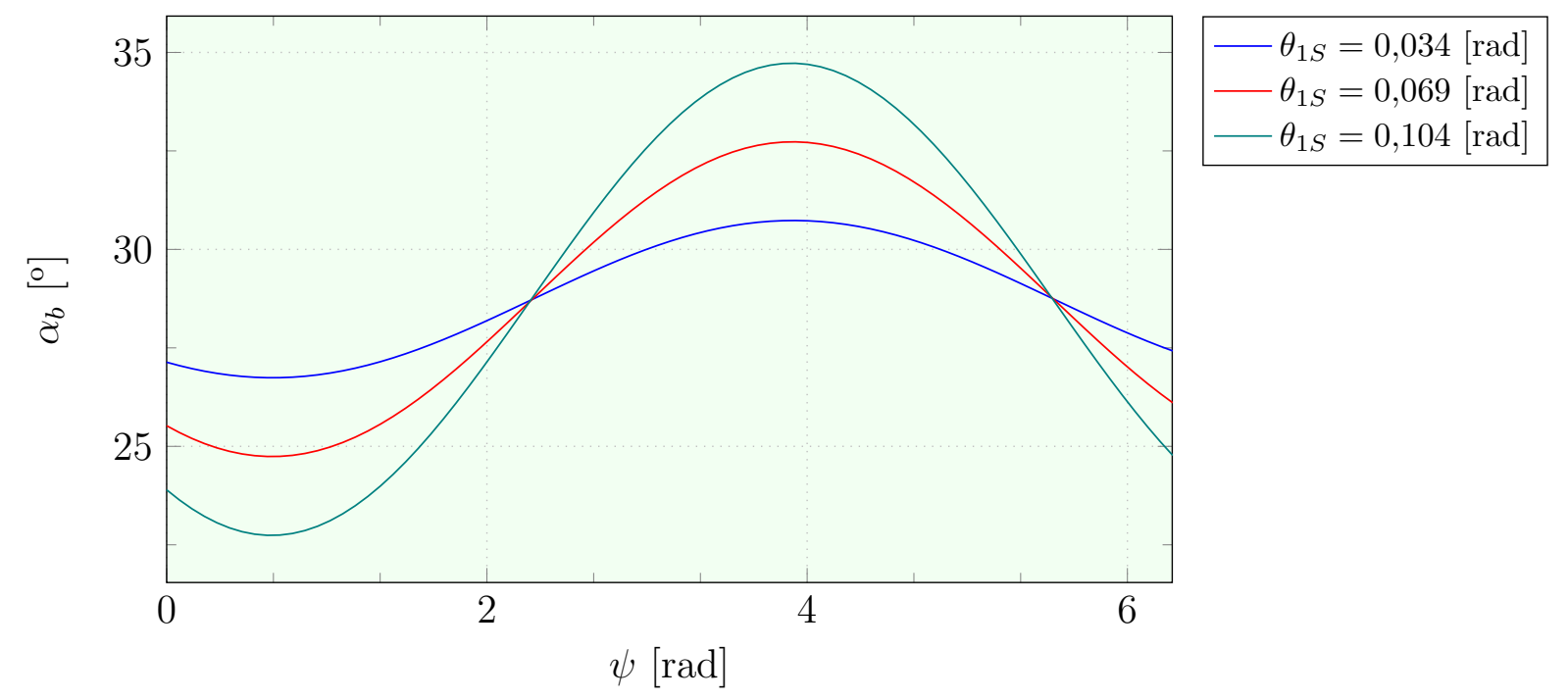

Figura 26: Ángulo de ataque de la pala en función de la posición azimutal.

\subsection{Conclusiones del capítulo}

La representación matemática del comportamiento del pararrotor en vuelo libre se realiza a partir de suponerlo como un sistema compuesto por tres sólidos 
rígidos (dos palas y un cuerpo cilíndrico), en el que interactúan acciones aerodinámicas, inerciales y gravitatorias, que sobre cada elemento componente tienen lugar. Se formularon las ecuaciones de la dinámica utilizando distintos sistemas de referencia, lo que permitió obtener expresiones matemáticas sencillas para las fuerzas y momentos, en las que se pueden identificar que parámetros son los que tienen mayor relevancia para caracterizar la dinámica del vuelo.

A partir de la utilización de las ecuaciones de Newton-Euler, en conjunto con las condiciones de ligadura cinemática entre los ángulos de Euler y las velocidades angulares expresadas en ejes cuerpo y con las ecuaciones de trayectoria del centro de masas del cuerpo del pararrotor, se obtuvo la formulación matemática de la dinámica del pararrotor en su forma canónica. Esta formulación tiene la ventaja de que permite su inmediata implementación en un código de resolución numérico, incorporando los modelos que se han presentado en el desarrollo de la sección, y también facilita el análisis de los efectos de cada elemento en la dinámica global del dispositivo.

A su vez, hipótesis simplificativas son introducidas en el modelo aerodinámico. Se considera que sobre el cilindro las fuerzas y momentos de origen aerodinámico son nulas, que la velocidad inducida por las palas es nula, que la componente de fuerza aerodinámica perpendicular a la dirección del viento relativo a la pala es nula, y que los coeficientes aerodinámicos son constantes.

Estas hipótesis permiten obtener un modelo descriptivo del comportamiento del pararrotor, a partir del desarrollo de hasta segundo orden de las expresiones de fuerzas y momentos.

Particularmente para el modelo adaptado al túnel de viento, los desarrollos de orden superior permiten señalar que:

- Los momentos de origen inercial son consecuencia de la aplicación de un ángulo de paso cíclico, ya sea lateral o longitudinal. Observando las ecuaciones (78), se evidencia que los momentos según $x_{A}$ e $y_{A}$ tienen relación lineal con el ángulo de paso cíclico, mientras que el momento según $z_{A}$ contiene términos cuadráticos.

- Los momentos inerciales se originan también debido a cambios temporales en la velocidad angular. Considerando términos de segundo orden, también contribuye el factor $\Omega^{2}(t)$.

- Los momentos aerodinámicos según $x_{A}$ e $y_{A}$ aparecen cuando se establece una ley de variación cíclica de las palas, mientras que el momento según $z_{A}$ depende del ángulo de paso colectivo y del régimen de descenso (a través del parámetro $\left.\mu_{z_{A}}\right)$ 
Estas observaciones permiten establecer que, mediante la variación cíclica del ángulo de paso de las palas, es posible controlar fuerzas y momentos de modo tal que se provoquen en el pararrotor modificaciones en las variables de estado.

La solución del sistema de ecuaciones reducido por las condiciones impuestas al arreglo en el túnel de viento se realizó tanto para el orden completo como para el desarrollo de segundo orden, arrojando resultados que difieren entre sí alrededor del $4 \%$. Estas diferencias pueden atribuirse al truncamiento en el desarrollo en series del modelo, en el que se descartan efectos de segundo orden para las acciones de control.

En cuanto al desarrollo en serie de Taylor, puede decirse que las expresiones obtenidas para las fuerzas y momentos brindan una herramienta de análisis cualitativo, a partir de la cual puede fundamentarse el comportamiento del pararrotor. A los efectos de cuantificar dicho comportamiento con mayor precisión, debe utilizarse el modelo completo, considerando incluso la adaptación del mismo mediante la implementación de modelos aerodinámicos de mayor sofisticación, en los que se consideren coeficientes de resistencia y sustentación no lineales.

La comparación entre los órdenes de magnitud de los momentos aerodinámicos y los inerciales permite desestimar estos últimos respecto a los primeros. El valor medio del momento aerodinámico aumenta junto al ángulo de paso cíclico, mientras que el momento inercial promediado en la vuelta es nulo. Esto puede explicarse refiriéndose a la ecuación (81) para los momentos aerodinámicos y la ecuación (78) para los momentos inerciales. En el caso de los momentos aerodinámicos, el promedio azimutal del desarrollo de primer orden expresado en (81) resulta distinto de cero, debido al factor $\sin ^{2} \psi$ que contiene la expresión. En cambio, para los momentos inerciales, si se considera que $\Omega(t)$ tiene una expresión armónica, al realizar la integral para calcular el promedio azimutal se tiene que en una vuelta completa este resulta nulo. 


\section{Estudio experimental del desplazamiento late- ral de decelerador aerodinámico a través de las variaciones cíclicas del paso de las palas}

\subsection{Introducción}

Para el desarrollo de los estudios experimentales se prepararon una serie de ensayos en base a la fabricación de un modelo de pararrotor con control de paso cíclico de las palas.

Se presentan a continuación las investigaciones experimentales realizadas en cuanto a la medición de fuerzas transmitidas por el pararrotor en función de variaciones cíclicas del ángulo de paso de las palas, a fin de validar, a través de ensayos, el modelo teórico elaborado.

\subsection{Descripción del modelo físico}

El modelo físico utilizado para los ensayos fue fabricado en base a un carenado cilíndrico, que representa el cuerpo del pararrotor, y dos palas acopladas al carenado mediante un rotor de un helicóptero de aeromodelismo del tipo T REX 450. Tanto el carenado cilíndrico como las palas del dispositivo se fabricaron mediante la técnica de impresión 3D, utilizando como material base el Acrinlonitrilo Butadieno Estireno (ABS), cuya densidad es de $1.050 \mathrm{~kg} / \mathrm{m}^{3}$.

También se fabricó en ABS el elemento de encastre y fijación de las palas, cumpliendo la función de unir las palas rectangulares y el portapalas del rotor. Como se muestra en la figura 27, las dimensiones de altura y diámetro del cilindro principal son iguales $(88 \mathrm{~mm})$, y la pala presenta una planta rectangular de $88 \mathrm{~mm}$ de envergadura y $138 \mathrm{~mm}$ de cuerda, siendo de las mismas dimensiones que las utilizadas en trabajos previos [20],[21].

A continuación se presentan los tensores de inercia del modelo, tanto para el cuerpo del pararrotor $(H)$ como para las palas $(b)$ : 


$$
\begin{aligned}
I_{H} & =\left[\begin{array}{ccc}
8,91 & 0 & 0 \\
0 & 8,91 & 0 \\
0 & 0 & 11,86
\end{array}\right] \cdot 10^{-4} \mathrm{kgm} \\
I_{b} & =\left[\begin{array}{ccc}
0,38 & 0 & 0 \\
0 & 3,25 & 0 \\
0 & 0 & 3,64
\end{array}\right] \cdot 10^{-4} \mathrm{kgm}
\end{aligned}
$$

En el cuadro siguiente se muestran las dimensiones características de las palas del modelo utilizado para los ensayos:

\begin{tabular}{ccc}
\hline \hline Envergadura $[\mathrm{m}]$ & Cuerda $[\mathrm{m}]$ & Superficie $\left[\mathrm{m}^{2}\right]$ \\
\hline 0,088 & 0,138 & 0,012 \\
\hline
\end{tabular}

Cuadro 3: Dimensiones de las palas

La figura siguiente indica gráficamente las dimensiones indicadas en el cuadro 3, junto a las dimensiones del cuerpo del pararrotor:
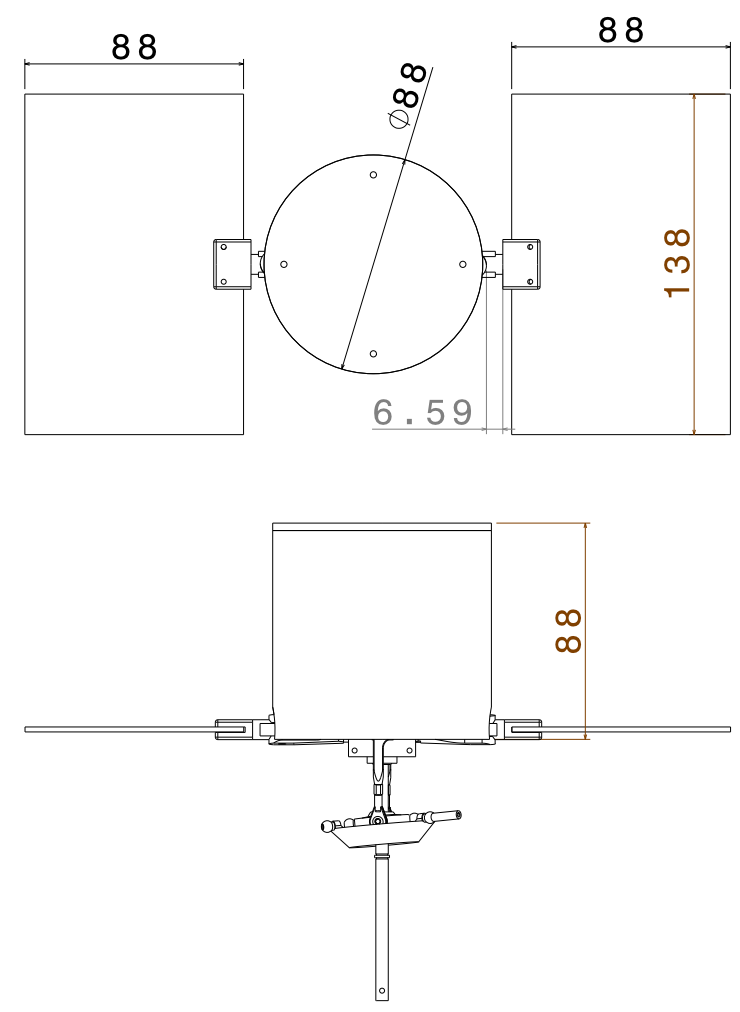

Figura 27: Dimensiones del modelo [mm] 
El rotor de helicóptero utilizado para el acople de las palas contiene un plato cíclico para el control del paso, que es accionado por una serie de servomecanismos ubicados en un chasis.

\subsection{Configuración experimental}

El pararrotor se ubicó dentro de un túnel de viento de sección de prueba rectangular. El túnel es de tipo "circuito cerrado", cuya sección de prueba tiene dimensiones de 1,4 m de ancho x $1 \mathrm{~m}$ de alto x 7,2 $\mathrm{m}$ de longitud. El aire es impulsado a partir de un motor de corriente continua de $50 \mathrm{Hp}$ de potencia, equipado con una hélice de paso fijo ajustable. Las velocidades se controlaron con un sistema electrónico, permitiendo ajustar la misma desde prácticamente 0 a $20 \mathrm{~m} / \mathrm{s}$. El túnel posee, además, un sistema de aletas para generar distintas configuraciones de flujo en la sección de prueba.

Para ubicar el pararrotor dentro de la sección de prueba se diseñaron una serie de bancadas. El primer diseño adoptado dispuso al pararrotor en forma de veleta, montado sobre el extremo de una barra vertical. En esta configuración, las vibraciones propias del dispositivo generaban interferencias mecánicas con las señales que se buscaba medir. Finalmente, se adoptó un diseño que se fijó al piso del túnel de viento y que contiene el eje de giro vertical mediante dos puntos, con lo que se redujo notablemente la interferencia mecánica del primer modelo de bancada. La bancada permite el giro del dispositivo alrededor del rotor por el efecto del viento, y también permite el giro alrededor del eje $z_{T}$, que es el eje alrededor del cual interesó medir la fuerza lateral que genera el modelo. El bloqueo del modelo (la relación entre el área frontal y el área de la sección de prueba) en el túnel de viento es menor a $5 \%$.

La figura 28 muestra, de modo esquemático, el soporte diseñado para el pararrotor.

La variación cíclica de las palas produce un momento sobre el cuerpo del pararrotor que es transmitido mediante la barra de aluminio vertical. Refiriéndose a la figura 28, se observa que esta barra (2) se encuentra empotrada sobre un par de rulemanes que permiten el giro alrededor de eje $x_{A}{ }^{1}$. La celda (4) se encuentra vinculada a la barra vertical mediante una rótula, por lo que el momento transmitido en es punto es nulo.

En la figura 29 se observa el modelo montado dentro del túnel, junto a los componentes necesarios para la ejecución del ensayo:

\footnotetext{
${ }^{1}$ de aquí en adelante se indica la dirección de la barra del sistema de medición en la dirección $x_{A}$, que es coincidente con la dirección $z_{T}$
} 


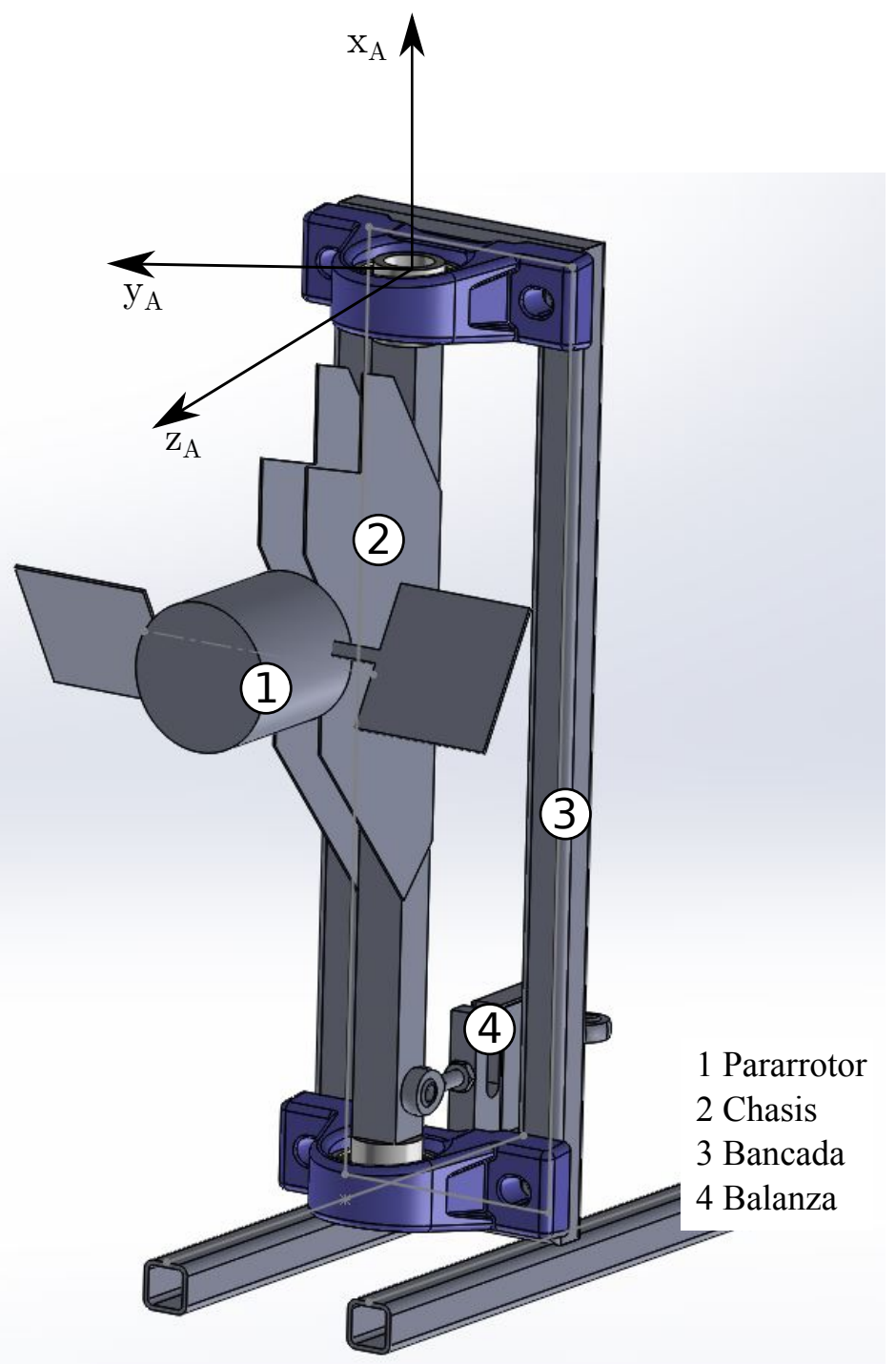

Figura 28: Esquema del soporte

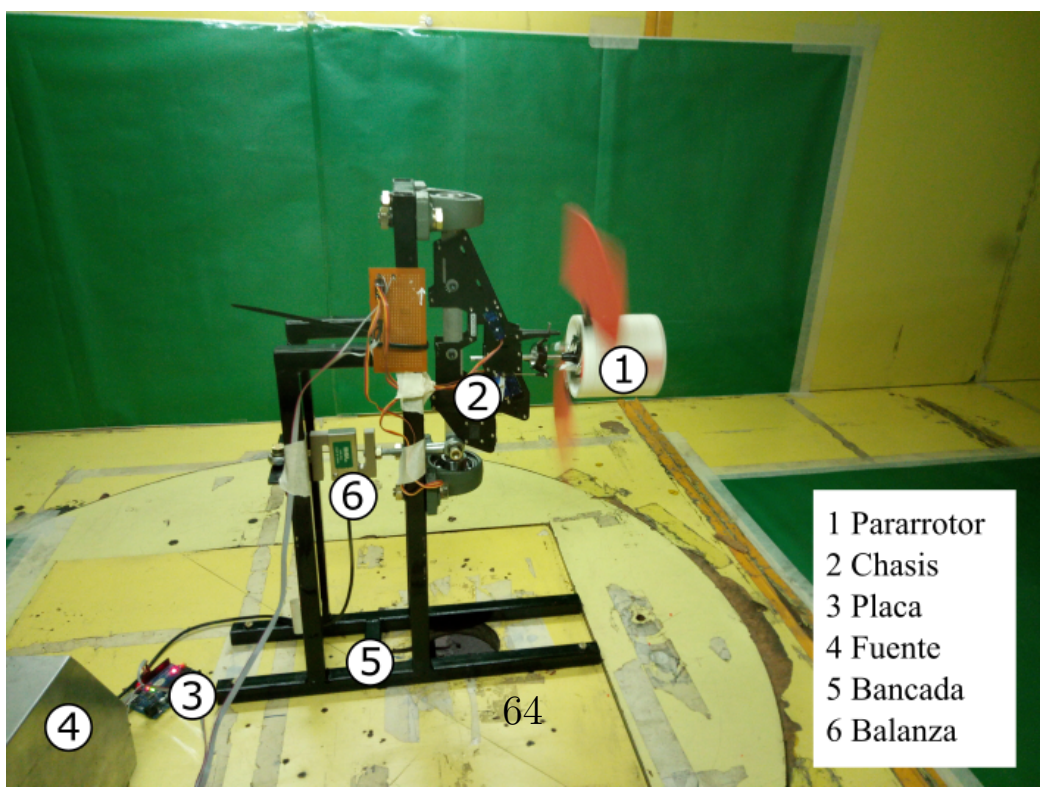

Figura 29: Montaje del modelo en el túnel de viento 


\subsubsection{Análisis estático de la bancada}

La bancada diseñada responde a las posibilidades constructivas con las que se contaron al momento de realizar el modelo experimental. Con el objetivo de establecer claramente las magnitudes medidas mediante el sistema diseñado, se realizó un análisis del equilibrio del sistema.

De esta manera se establece que magnitud se está midiendo en la celda de carga.

Se adopta como modelo bajo estudio a la barra vertical que sostiene al chasis (ver figuras 28 y 29), ya que es sobre esta barra donde concurren las fuerzas generadas por el pararrotor, los rodamientos que la sostienen y la celda de carga.

El desarrollo presentado en el apartado anterior permite obtener el momento generado sobre el centro de masas del pararrotor.

Dado que se busca comparar el modelo teórico desarrollado con resultados experimentales, es necesario presentar una ecuación que habilite tal comparación.

Esta ecuación se obtiene simplemente trasladando el momento y la fuerza que genera el pararrotor respecto a su centro de masas. Se debe tener en cuenta que la fuerza generada en el centro de masas del pararrotor genera un momento respecto al punto de medición que es necesario tener en cuenta. La figura 30 muestran los puntos de referencia utilizados sobre el pararrotor para la definición de las magnitudes necesarias.

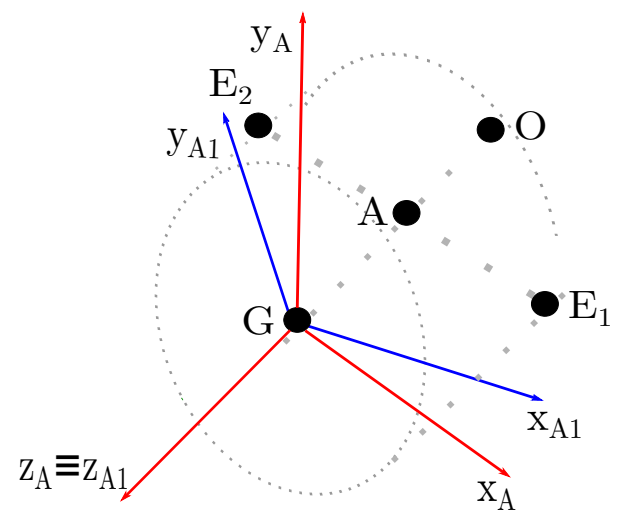

Figura 30: Puntos de referencia para la determinación de fuerzas y momentos sobre el pararrotor

En la figura 30 se identifican los siguientes puntos de referencia:

- $E_{1}$ y $E_{2}$, puntos de acoplamiento de las palas con el cilindro del pararrotor, 
- A, punto de coincidencia del eje del pararrotor con el eje de las palas,

- G, centro de masas del pararrotor,

- O, punto de acoplamiento del pararrotor al sistema de media.

Valiéndose de la figura 31 se puede deducir la expresión para la componente de fuerza que se registra mediante la celda de carga.

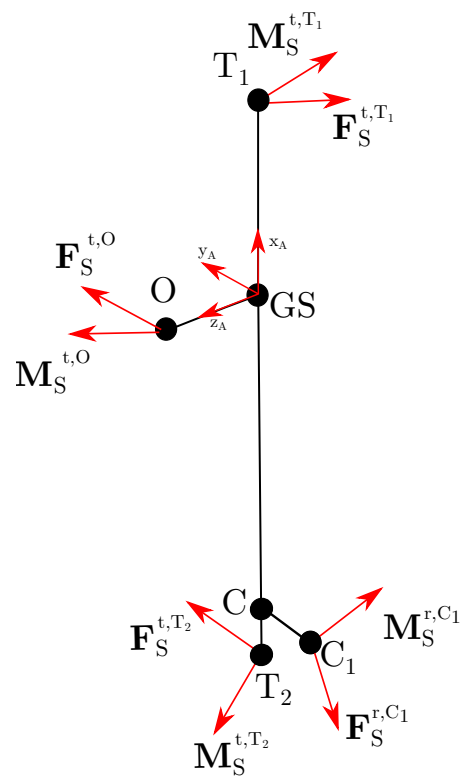

Figura 31: Diagrama de fuerzas transmitidas a la barra del sistema experimental

Al desplazar el eje respecto al cual se miden momentos desde el punto $A$, perteneciente al pararrotor, al punto $O$, que vincula el pararrotor con el mecanismo de medida, la fuerza $\mathbf{F}^{t}$ contribuye con un momento igual a $\mathbf{O A} \wedge \mathbf{F}^{t}$ (ver figura 31).

La ecuación de equilibrio rotacional sobre la barra respecto al centro de masas de la barra (punto GS) puede plantearse, de manera general, como sigue:

$$
\begin{gathered}
\overbrace{\mathbf{M}_{H}^{t, A}+\mathbf{O A} \wedge \mathbf{F}^{t}}^{\mathbf{M}_{S}^{t, O}}+\mathbf{G S O} \wedge \mathbf{F}_{S}^{t, O}+\mathbf{M}_{S}^{t, T_{1}}+\mathbf{G S T}_{1} \wedge \mathbf{F}_{S}^{t, T_{1}} \\
\quad+\mathbf{M}_{S}^{t, T_{2}}+\mathbf{G S T}_{2} \wedge \mathbf{F}_{S}^{t, T_{2}}+\mathbf{M}_{S}^{r, C_{1}}+\mathbf{G S C}_{1} \wedge \mathbf{F}_{S}^{r, C_{1}}=0
\end{gathered}
$$

Donde los superíndices $t$ y $r$ indican la naturaleza de la fuerza o el momento. El superínice $t$ indica transmisión hacia el elemento sobre el que se plantea el balance de fuerzas y momentos, y el superíndice $r$ indica reacción por parte del 
elemento sobre el que se plantea el balance. Los superíndices $T_{i}, O, A, C_{i}$ indican los puntos de aplicación. Los subíndices indican el cuerpo sobre el que se aplican $(S$ se utiliza para representar la barra). Para simplificar la ecuación (89), se asume que los puntos $T_{1}$ y $T_{2}$ son rodamientos cuyo eje coincide con el eje $x_{A}$ (no transmiten momentos según esa dirección) y que el punto $C_{1}$ es una rótula. Con estas hipótesis, se puede plantear la proyección de la ecuación (89) según $x_{A}$ en el punto GS como sigue:

$$
M_{S, x_{A}}^{t, O}+G S O_{z_{A}} \cdot F_{S, y_{A}}^{t, O}+C C_{1_{y_{A}}} \cdot F_{S, z_{A}}^{r, C_{1}}=0
$$

Considerando que $M_{S}^{t, O}=-M_{H}^{r, O}$ y $F_{S}^{t, O}=-F_{H}^{r, O}$, pueden asignarse a estas magnitudes las expresiones desarrolladas previamente. De la ecuación (90) se puede obtener la expresión para la fuerza que registra la celda de carga, despejando $F_{S, z_{A}}^{r, C_{1}}$. Llamando $F=-F_{S, z_{A}}^{r, C_{1}}, G S O_{z_{A}}=l_{O}$ y $C C_{1 y_{A}}=-l_{C_{1}}$ resulta:

$$
F=-\frac{l_{O}}{l_{C_{1}}} F_{S, y_{A}}^{t, O}-M_{S, x_{A}}^{t, O} \frac{1}{l_{C_{1}}}
$$

A partir de la ecuación (91) se compararon los resultados del subsistema correspondiente del modelo teórico con el desarrollo experimental.

\subsection{Parámetros medidos e instrumentación}

Los parámetros medidos durante los ensayos fueron:

- Velocidad de la corriente, $\mathbf{U}_{W}$,

- Velocidad angular del pararrotor, $\Omega$,

- Fuerza medida en la balanza, $F$,

- Ángulos de paso colectivo y cíclico longitudinal de las palas, $\theta_{0}$ y $\theta_{1 S}$

- Temperaturas de bulbo seco y bulbo húmedo, $T_{b s}$ y $T_{b h}$,

- Presión atmosférica, $P_{a t m}$.

Para la medición de estos parámetros se utilizaron los siguientes instrumentos: 


\begin{tabular}{|c|c|c|c|c|c|}
\hline Parámetro & Instrumento & Marca & Modelo & Rango & Resolución \\
\hline $\begin{array}{l}\text { Velocidad de la } \\
\text { corriente }\end{array}$ & $\begin{array}{l}\text { Anemómetro de hilo } \\
\text { caliente }\end{array}$ & Extech & 407123 & $0,2-20 \mathrm{~m} / \mathrm{s}$ & $0,1 \mathrm{~m} / \mathrm{s}$ \\
\hline $\begin{array}{l}\text { Velocidad angu- } \\
\text { lar }\end{array}$ & $\begin{array}{l}\text { Lámpara } \\
\text { boscópica }\end{array}$ & Lutron & DT-2239A & 10-10000 RPM & $1 \mathrm{RPM}$ \\
\hline Fuerza & Celda de carga tipo S & BSL & MTS-1 & $0-10 \mathrm{~N}$ & $2 \mathrm{mV} / \mathrm{V}$ \\
\hline $\begin{array}{l}\text { Ángulo de las } \\
\text { palas }\end{array}$ & Goniómetro digital & RC Logger & $\begin{array}{l}4000 \\
2 \mathrm{RCD}\end{array}$ & 0 a $90^{\circ}$ & $0,1^{\circ}$ \\
\hline Temperatura & $\begin{array}{l}\text { Termómetro bulbo se- } \\
\text { co y húmedo }\end{array}$ & - & - & $\begin{array}{l}\mathrm{BH}-25^{\circ} \text { a } 35^{\circ} \mathrm{C}, \\
\mathrm{BS} 0^{\circ} \mathrm{C} \text { a } 100^{\circ} \mathrm{C}\end{array}$ & \\
\hline Presión & Barómetro digital & Druck & DPI740 & $\begin{array}{l}75000 \text { a } 115000 \\
\text { Pa. }\end{array}$ & $1 \mathrm{~Pa}$. \\
\hline
\end{tabular}

Cuadro 4: Instrumentos utilizados

\subsubsection{Medición de la fuerza en la balanza}

Para poder obtener resultados experimentales relativos al torque generado por la variación cíclica del paso de las palas, se midió y adquirió la fuerza medida por la balanza. Para esto se utilizó una celda de carga tipo S dispuesta de la manera que se indica en la figura 28 e indicada con el número 5 en la figura 29. Un esquema de esta celda se muestra en la figura (32).

A continuación se tabulan las especificaciones de la celda de carga utilizada:

\begin{tabular}{ccc}
\hline \hline Dato técnico & Unidad & Valor \\
\hline Sensibilidad & $\mathrm{mV} / \mathrm{V}$ & $2 \pm 0,01$ \\
Rango & $\mathrm{kg}$ & $0-5$ \\
Material & - & Acero inoxidable \\
Tipo de rosca & - & M8x1,25 \\
Altura (B) & $\mathrm{mm}$ & 64 \\
Ancho (C) & $\mathrm{mm}$ & 51 \\
Profundidad (A) & $\mathrm{mm}$ & 12,7 \\
\hline
\end{tabular}

Cuadro 5: Datos técnicos celda de carga

Esta celda absorbe la carga que el pararrotor transmite a través del sistema de medida.

La señal que genera la celda de carga es acondicionada antes de ser adquirida por un programa de adquisición desarrollado a propósito del ensayo. El acondicionador de señal convierte la señal que envía la celda de carga y la transforma en una corriente, que es enviada al programa de adquisición a través de una placa adquisidora externa. Esta señal es almacenada con una frecuencia de $1 \mathrm{kHz}$ en un fichero que posteriormente es utilizado para el análisis y procesamiento de los datos. 


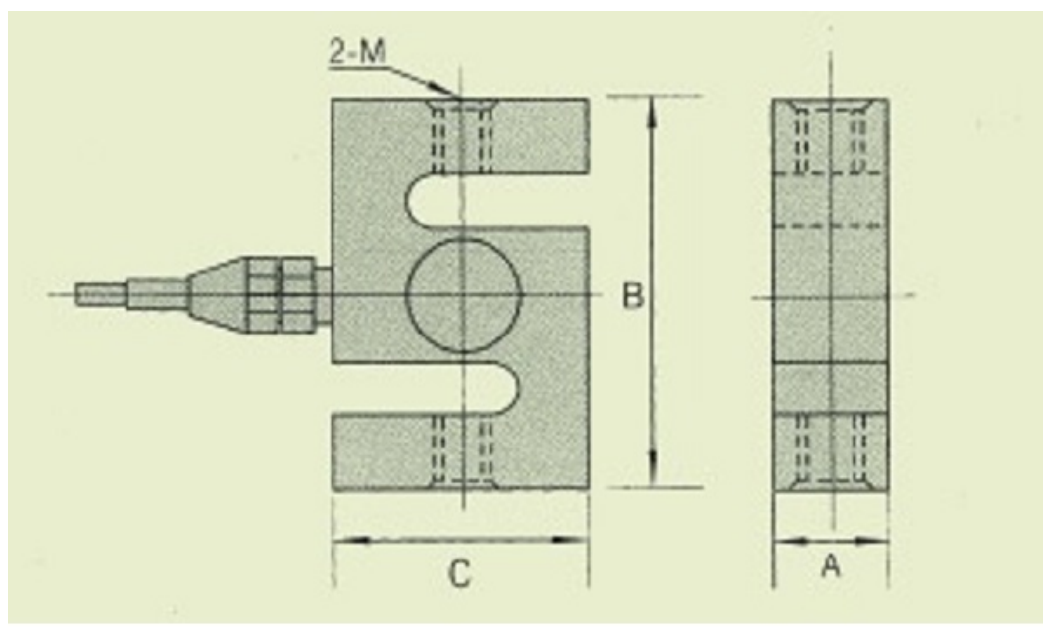

Figura 32: Esquema de la celda

Los registros de la velocidad media de la corriente, $\mathbf{U}_{W}$ y de la velocidad de giro del pararrotor, $\Omega$, se realizan manualmente para cada una de las corridas del ensayo.

Los parámetros atmosféricos de referencia son relevados al inicio y al final de la realización de los ensayos, para determinar posteriormente la densidad del aire.

\subsection{Control del paso cíclico de las palas}

El sistema de control de paso cíclico de las palas es llevado a cabo por un sistema de tres servomecanismos colocados en un chasis que facilita la vinculación espacial entre los servomecanismos y los puntos de anclaje del plato cíclico del rotor. A continuación se presentan los datos técnicos de los mismos:

\begin{tabular}{ccc}
\hline \hline Dato técnico & Unidad & Valor \\
\hline Peso & $\mathrm{kg}$ & 0,009 \\
Tensión de operación & $\mathrm{V}$ & $3-6$ \\
Torque & $\mathrm{kg} .-\mathrm{cm}$ & 1,6 \\
\hline
\end{tabular}

Cuadro 6: Datos técnicos de los servomecanismos

La señal a los servomecanismos es enviada a través de una placa Arduino (®) UNO, que media entre los servomecanismos y la CPU utilizada para el control y la adquisición de los datos. La alimentación de los servomecanismos es provista por una fuente externa.

Se diseñó un instrumento virtual con una interfaz gráfica (figura 33) uti- 
lizando un lenguaje de programación gráfico. Este instrumento permitió establecer, para cada uno de los servomecanismos, la posición angular que deben tomar para que cada una de las palas del pararrotor se ubiquen en el ángulo deseado. Mediante esta interfaz se ingresaron los ángulos colectivos y cíclicos deseados, que son procesados por el programa desarrollado para enviar la señal a cada uno de los servomecanismos.

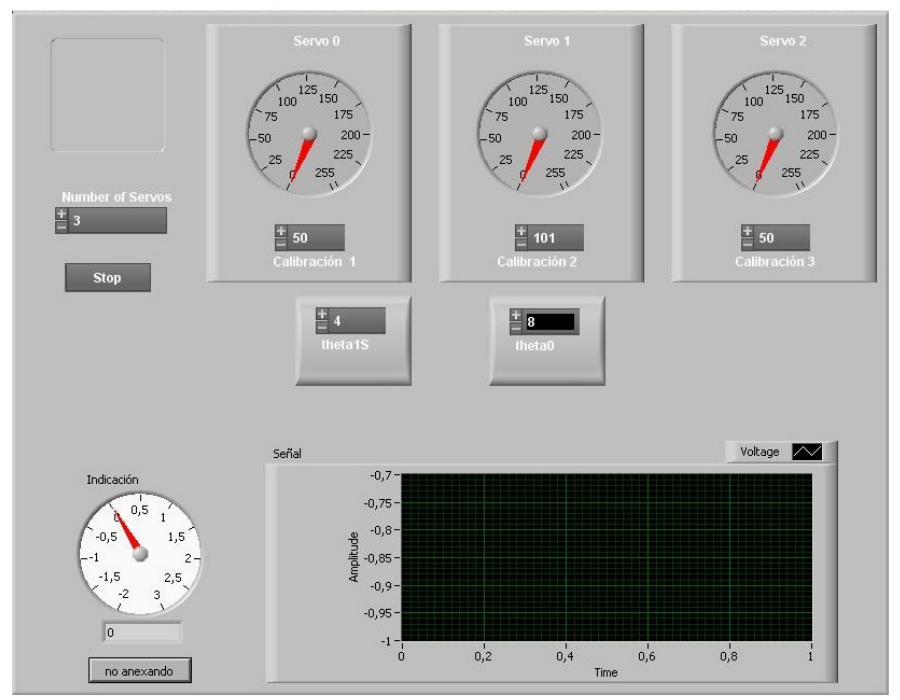

Figura 33: Interfaz del sistema de adquisición

La calibración de este sistema se realizó fuera del túnel de viento. Para esto, se realizó un procedimiento que se detalla a continuación:

1. Definición del plano de ángulo colectivo nulo: con el eje del rotor en forma vertical, se midió en tres ángulos de azimut distintos del rotor (indicados como testigos en la figura 34) el ángulo de paso de las palas. Mediante el programa de control desarrollado se buscó que el ángulo de las palas, cuando las varillas que controlan el ángulo del portapalas se encuentran sobre dichos puntos, sea 0 .

2. Obtención de la curva de calibración: para obtener la relación entre el valor numérico ingresado al programa y el ángulo que toman los servomecanismos, se construyó una tabla a partir de determinar el plano del plato para el cuál las palas tomaban valores de $2^{\circ}, 4^{\circ}, 6^{\circ}$ y $8^{\circ}$. De esta manera, se vinculó el valor numérico del programa asociado a la posición angular de cada servomecanismo con un ángulo de paso de las palas.

En base a esto, y por medio de un ajuste por mínimos cuadrados, se obtienen las ecuaciones que se programan en el software para controlar cada uno de los servomecanismos. 


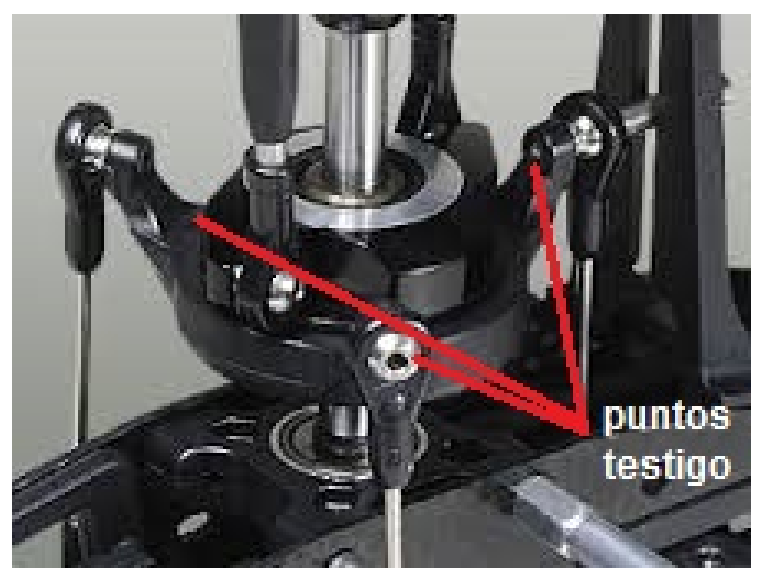

Figura 34: Puntos de calibración del plano "0"

3. Calibración del paso cíclico: para obtener la ecuación de calibración del paso cíclico se procedió de manera similar a la del paso colectivo, colocando las palas en $\psi=0 \mathrm{rad}$ y $\psi=\pi \mathrm{rad}$, y configurando los servomecanismos de manera que las palas tomen los ángulos que se listan a continuación, siempre para $\theta_{0}=0$ :

\begin{tabular}{cc}
\hline \hline$\theta_{1}(\mathrm{rad})$ & $\theta_{2}(\mathrm{rad})$ \\
\hline 0,034 & $-0,034$ \\
0,069 & $-0,069$ \\
0,104 & $-0,104$ \\
0,14 & $-0,14$ \\
\hline
\end{tabular}

Cuadro 7: Ángulos de paso cíclico para la calibración

Para todas estas combinaciones se realizó la vinculación entre el valor numérico ingresado al programa para cada servomecanismo y el ángulo de paso que tomaba cada pala.

\subsection{Ensayos}

\subsubsection{Medición de la fuerza en la balanza}

Para el modelo físico descripto en la sección 4.2 se realizaron una serie de ensayos en el túnel de viento según los parámetros establecidos en el siguiente cuadro: 


\begin{tabular}{cc}
\hline \hline$\theta_{0}[\mathrm{rad}]$ & $\theta_{1 S}[\mathrm{rad}]$ \\
\hline \multirow{3}{*}{0,034} & 0,034 \\
& 0,069 \\
& 0,104 \\
& 0,139 \\
& 0,174 \\
\hline \multirow{3}{*}{069} & 0,034 \\
& 0,069 \\
& 0,104 \\
& 0,139 \\
& 0,174 \\
\hline & 0,034 \\
0,104 & 0,069 \\
& 0,104 \\
& 0,139 \\
& 0,174 \\
\hline
\end{tabular}

Cuadro 8: Ángulos de ensayo

Para cada una de las configuraciones del cuadro 8 se realizaron mediciones de la fuerza en la balanza, de la velocidad angular del modelo y la velocidad de la corriente de viento, a fin de cuantificar el valor de la fuerza lateral sobre la balanza generada por la variación cíclica del ángulo de paso de las palas.

\subsubsection{Resultados}

En este apartado se presentan los resultados obtenidos a partir de los ensayos en el túnel de viento. Las configuraciones ensayadas son las que se indican en el cuadro 8, para las cuales se midieron la velocidad de la corriente libre, la velocidad angular del pararrotor y la fuerza lateral generada.

Se presentan por un lado los resultados obtenidos para la velocidad angular, junto a su comparación con el modelo teórico, y por otro los resultados de la medición de la fuerza lateral, en conjunto con la estimación teórica del modelo desarrollado.

\subsubsection{Procesamiento de los resultados de medición de la velocidad an- gular}

El primer parámetro que se mide en el ensayo es la velocidad angular que alcanza el pararrotor. A continuación se muestran los valores medios de los 
resultados obtenidos para los casos indicados en el cuadro 9:

\begin{tabular}{cccc}
\hline \hline$\theta_{0}(\mathrm{rad})$ & $\theta_{1 S}(\mathrm{rad})$ & $\mathrm{V}_{Z}(\mathrm{~m} / \mathrm{s})$ & $\Omega_{0}(\mathrm{rad} / \mathrm{s})$ \\
\hline \multirow{5}{*}{0,034} & 0,034 & 5,3 & 94,1 \\
& 0,069 & 5,3 & 92,7 \\
& 0,104 & 5,3 & 90,1 \\
& 0,139 & 5,4 & 88,6 \\
& 0,174 & 5,3 & 87,1 \\
\hline \multirow{5}{*}{0,069} & 0,034 & 5,3 & 107,44 \\
& 0,069 & 5,3 & 107,0 \\
& 0,104 & 5,3 & 106,8 \\
& 0,139 & 5,4 & 104,3 \\
& 0,174 & 5,3 & 103,9 \\
\hline \multirow{5}{*}{0,104} & 0,034 & 5,3 & 110,5 \\
& 0,069 & 5,3 & 107,6 \\
& 0,104 & 5,4 & 107,1 \\
& 0,139 & 5,3 & 106,8 \\
& 0,174 & 5,4 & 105,9 \\
\hline
\end{tabular}

Cuadro 9: Resultados

En trabajos anteriores [20], se realizaron ensayos con ángulo de paso colectivo constante y ángulo de paso cíclico nulo. Estos ensayos son utilizados como referencia inicial para caracterizar el desempeño de los ensayos realizados en el presente trabajo. El siguiente cuadro muestra la comparación del coeficiente de velocidades, definido en esta tesis como $\mu_{z_{A}}=U_{0}\left(\Omega_{0} \cdot R_{b}\right)^{-1}$, entre los ensayos publicados en el trabajo de referencia y los realizados para el presente trabajo:

\begin{tabular}{|c|c|c|c|}
\hline$\overline{\theta_{0}(\mathrm{rad})}$ & $\bar{~}_{\mu_{z_{A}}}$ referencia & 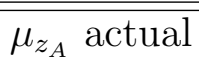 & diferencia (\%) \\
\hline 0,034 & 0,35 & 0,40 & 12 \\
\hline 0,069 & 0,32 & 0,34 & 6 \\
\hline 0,104 & 0,29 & 0,32 & 9 \\
\hline
\end{tabular}

Cuadro 10: Comparación de resultados con trabajo de referencia [20]

La diferencia para el coeficientes de velocidades $\mu_{z_{A}}$, en términos porcentuales, resulta aceptable. Pueden atribuirse las discrepancias a las distintas configuraciones experimentales en cada trabajo. 


\subsection{Comparación con el modelo teórico}

La comparación entre los resultados experimentales y los obtenidos con el modelo teórico se muestran en el siguiente cuadro:

\begin{tabular}{cccc}
\hline \hline$\theta_{0}(\mathrm{rad})$ & $\theta_{1 S}(\mathrm{rad})$ & $\Omega_{\text {exp }}(\mathrm{rad} / \mathrm{s})$ & $\Omega(\mathrm{rad} / \mathrm{s})$ \\
\hline \multirow{5}{*}{0,034} & 0,034 & 94,1 & 74,4 \\
& 0,069 & 92,7 & 74,4 \\
& 0,104 & 90,1 & 74,4 \\
& 0,139 & 88,6 & 74,4 \\
& 0,174 & 87,1 & 74,4 \\
\hline \multirow{5}{*}{0,069} & 0,034 & 107,44 & 77,6 \\
& 0,069 & 107,0 & 77,6 \\
& 0,104 & 106,8 & 77,6 \\
& 0,139 & 104,3 & 77,6 \\
& 0,174 & 103,9 & 77,6 \\
\hline \multirow{5}{*}{0,104} & 0,034 & 110,5 & 80,8 \\
& 0,069 & 107,6 & 80,8 \\
& 0,104 & 107,1 & 80,8 \\
& 0,139 & 106,8 & 80,8 \\
& 0,174 & 105,9 & 80,8 \\
\hline
\end{tabular}

Cuadro 11: Resultados

Se observa que existe una diferencia considerable (del orden de 30\%) entre las mediciones en el túnel de viento y lo que predice el modelo teórico. Además, el modelo no predice la variación de la velocidad angular con el ángulo de paso cíclico.

\subsubsection{Procesamiento de los resultados de medición de fuerza en la balanza}

Los datos adquiridos por la balanza se muestran en la siguiente figura. La misma (figura 35) presenta la evolución de la tensión (indicada en $\mathrm{mV}$ en el eje vertical) contra el tiempo.

Como se observa, el patrón que presenta no se asocia de manera directa a lo que hipotéticamente se espera en base a lo analizado en el capítulo 3, sino que, sobre la base de una evolución periódica de primer orden, aparecen componentes de mayor frecuencia que introducen ruidos. El desfasaje que aparece entre las distintas señales se debe a que según el ángulo de paso cíclico se tienen distintas velocidades angulares. 


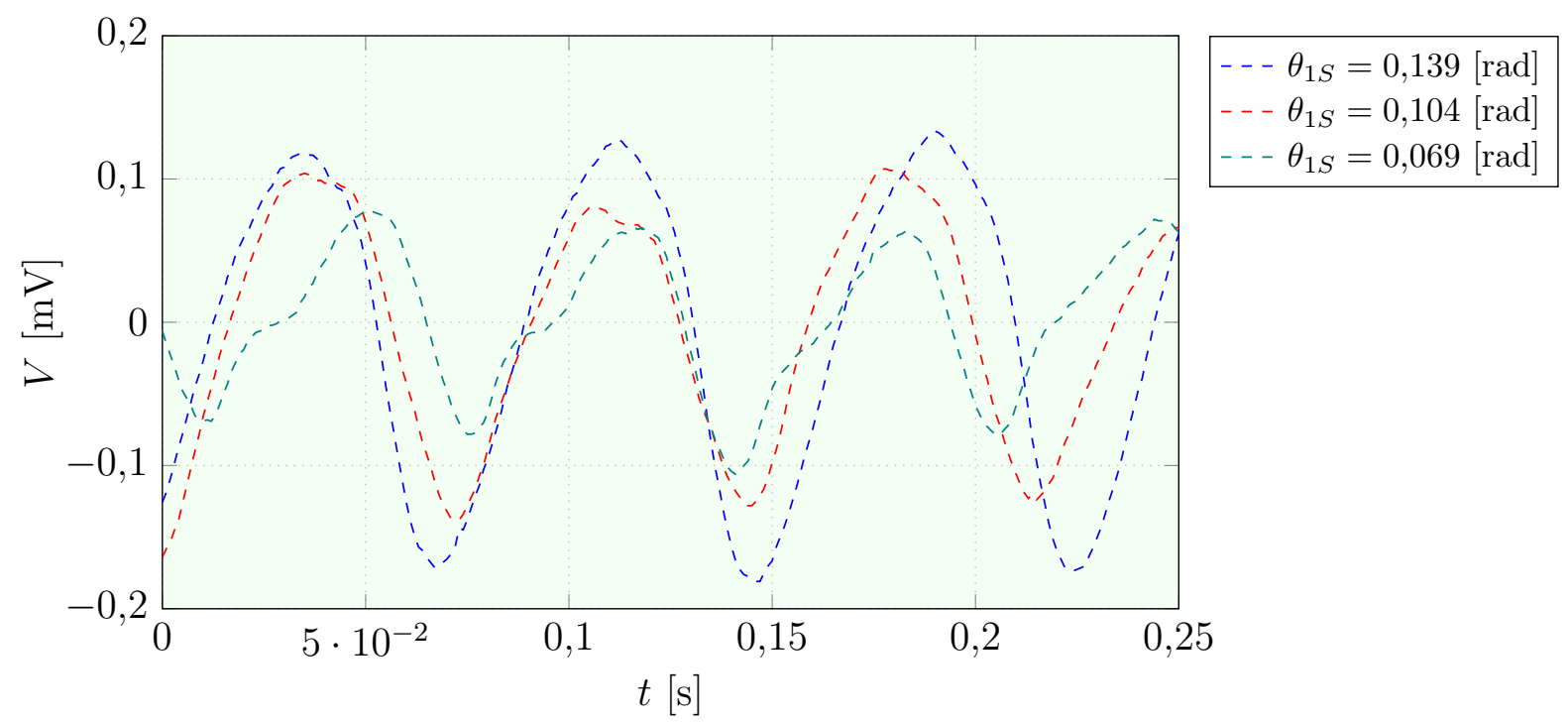

Figura 35: Medida registrada en la balanza para un ángulo de paso colectivo 0,104 rad.

Con el objetivo de obtener indicaciones comparables para validar el modelo teórico, el registro realizado mediante el adquisidor de fuerza es procesado digitalmente.

Se realiza un modelado de los datos experimentales ajustando los mismos por medio de una combinación lineal de coeficientes a determinar y una serie de funciones base de modo que el error experimental asociado a la medición de la fuerza en la balanza (variable dependiente) se minimice. En términos matemáticos se puede formular la siguiente expresión:

$$
\chi^{2}\left(a_{1}, a_{2}, \ldots, a_{n}\right)=\sum_{i=1}^{N} \frac{\left(y_{i}-\sum_{r=0}^{n} a_{r} \tau_{r}\left(x_{i}\right)\right)^{2}}{\sigma_{i}^{2}}
$$

Donde:

- $\chi$ es el error cuadrático medio del ajuste experimental,

- $a_{r}$ es el coeficiente de ajuste de datos experimentales,

- $y_{i}$ es la distribución de datos experimentales,

- $\tau_{r}$ es la familia de funciones base para el ajuste de los datos,

- $x_{i}$ es la variable dependiente de la familia de funciones de ajuste,

- $\sigma_{i}$ es el error experimental. 
Dado el comportamiento que presentan los datos experimentales se selecciona una base de funciones trigonométricas para realizar el modelado de los datos. La base de funciones ortogonales es $\{1, \cos x, \sin x\}$, limitando el ajuste a lo que se conoce como serie de Fourier discreta de primer orden.

Para cada caso de ensayo, se calcularon los coeficientes $a_{i}$ para ajustar la ecuación:

$$
F_{x_{A}}^{e x p}=a_{0}+a_{1} \sin \psi+a_{2} \cos \psi
$$

En el gráfico siguiente se muestran las interpolaciones para los resultados obtenidos tomando dos configuraciones del plato cíclico. Los gráficos representan la evolución del momento en media vuelta del rotor (se resta a esta señal el valor promedio del coeficiente de momento a efectos de visualizar mejor el cambio en la magnitud a lo largo de la vuelta).

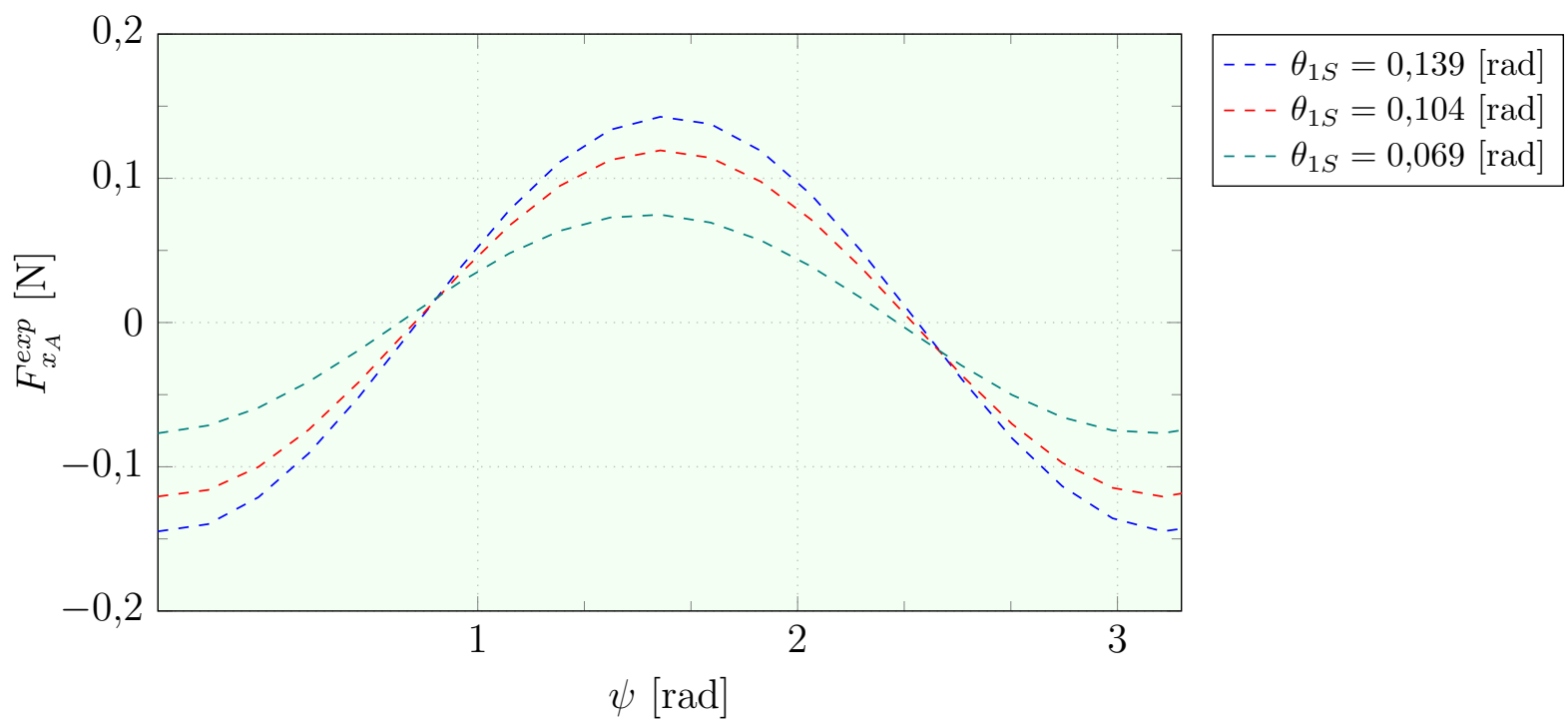

Figura 36: Modelo experimental de la fuerza registrada en la balanza para un ángulo de paso colectivo 0,139 rad. 


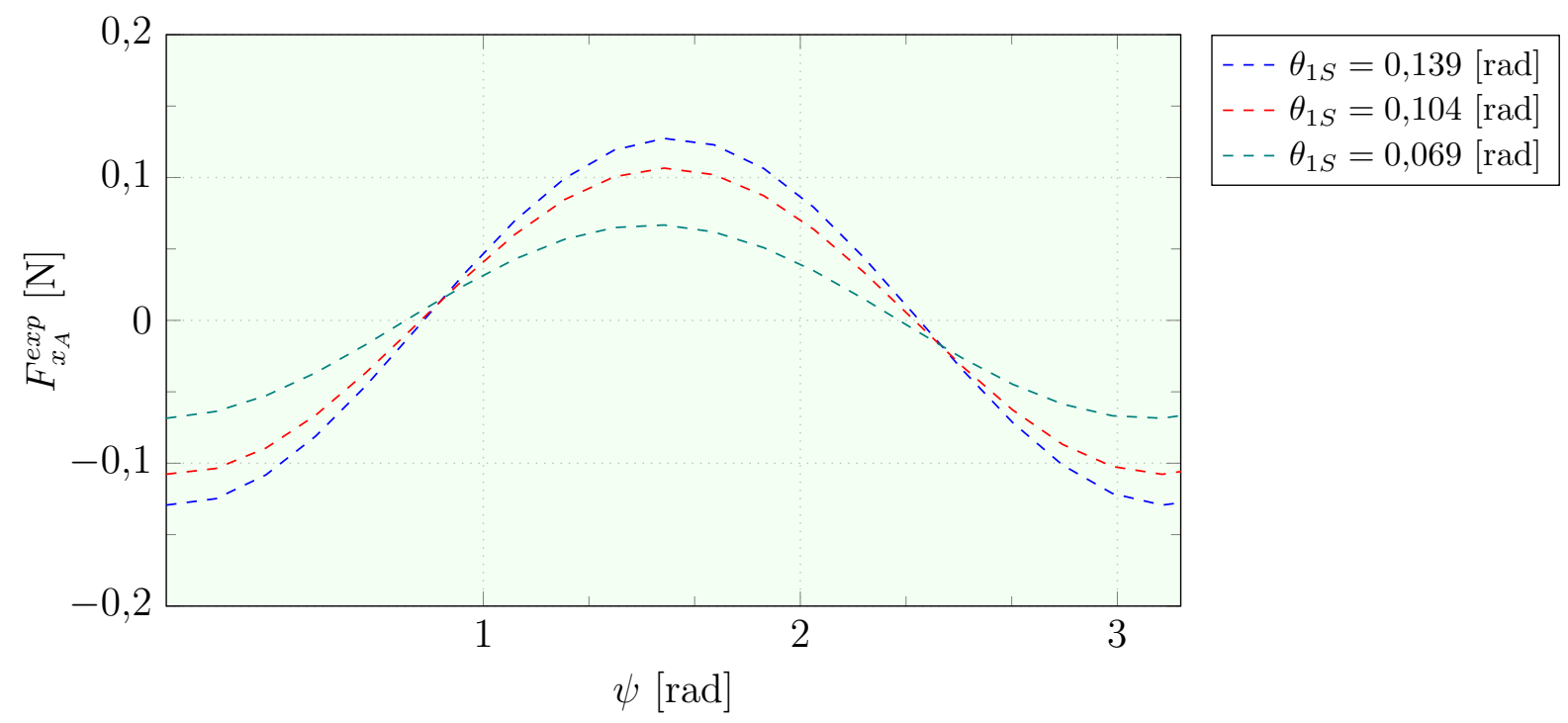

Figura 37: Modelo experimental de la fuerza registrada en la balanza para un ángulo de paso colectivo 0,104 rad.

\subsection{Comparación con el modelo teórico}

La comparación entre los resultados obtenidos de los ensayos experimentales y el modelo teórico en el túnel de viento es una herramienta de diagnóstico que permite determinar el grado de aproximación de las hipótesis implementadas. Los resultados teóricos de fuerzas y momentos son reemplazados en la ecuación (91) para obtener las figuras 38 a 43.

La primera observación que se hace es que el modelo teórico se ajusta a los resultados experimentales. Se observan diferencias máximas del orden del $13 \%$, que disminuyen a menos del $1 \%$ a medida que se incrementa $\theta_{1 S}$.

Con la contrastación positiva entre el modelo teórico y los resultados experimentales, se puede dar validez a los resultados cuantitativos presentados en el apartado 3.11.3.

\subsection{Incertidumbre de las mediciones}

Para la estimación de las incertidumbres asociadas a la realización de los experimentos se toma como guía el trabajo [32]. Este tratamiento de los resultados tiene como objetivo estimar la incertidumbre de los mismos, considerando la distribución propia de los distintos valores obtenidos (incertidumbre tipo A) como así también la incertidumbre asociada a los distintos instrumentos de medición y el 


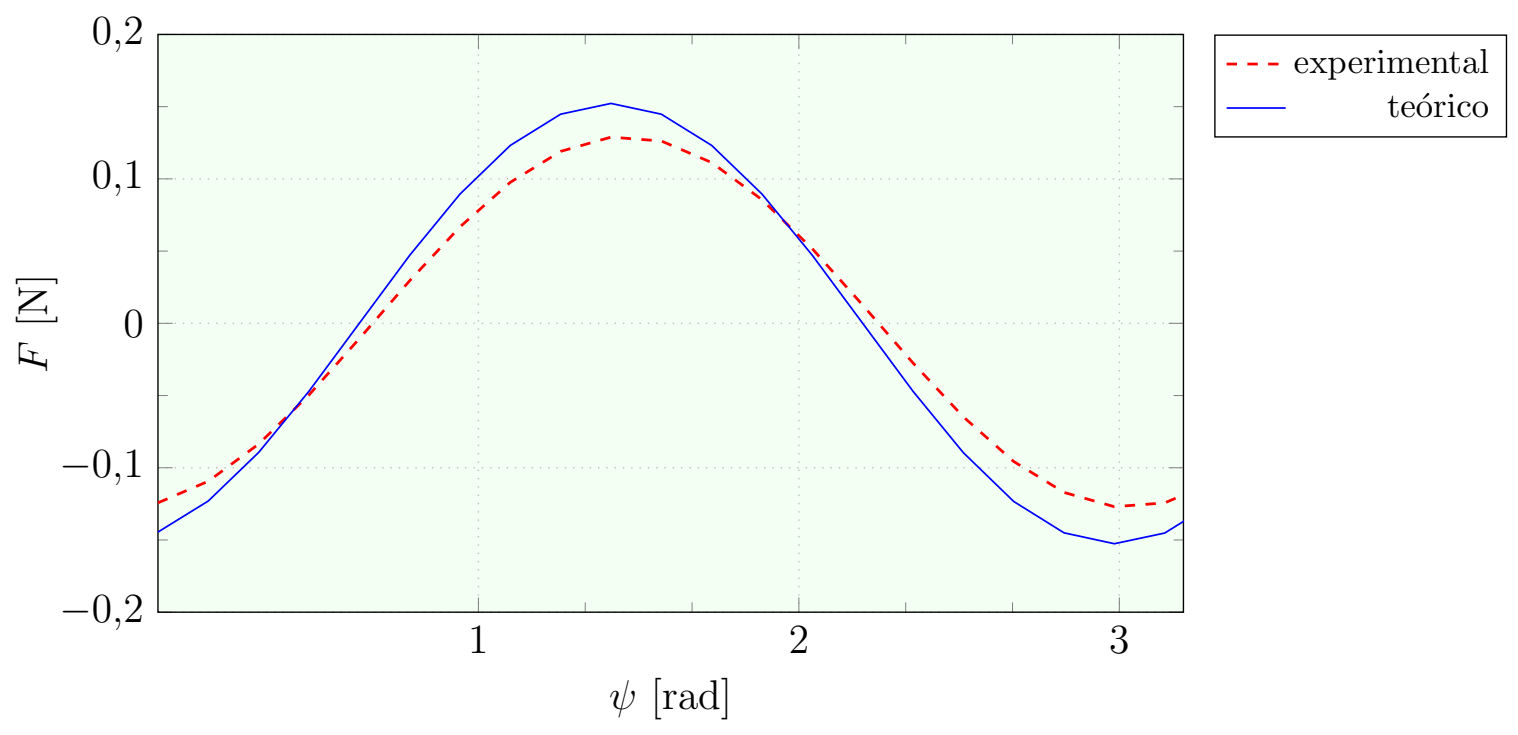

Figura 38: Comparación entre el modelo experimental de la fuerza registrada en la balanza y el modelo teórico para ángulos de paso $\theta_{0}=0,139 \mathrm{rad} \theta_{1 S}=0,139 \mathrm{rad}$.

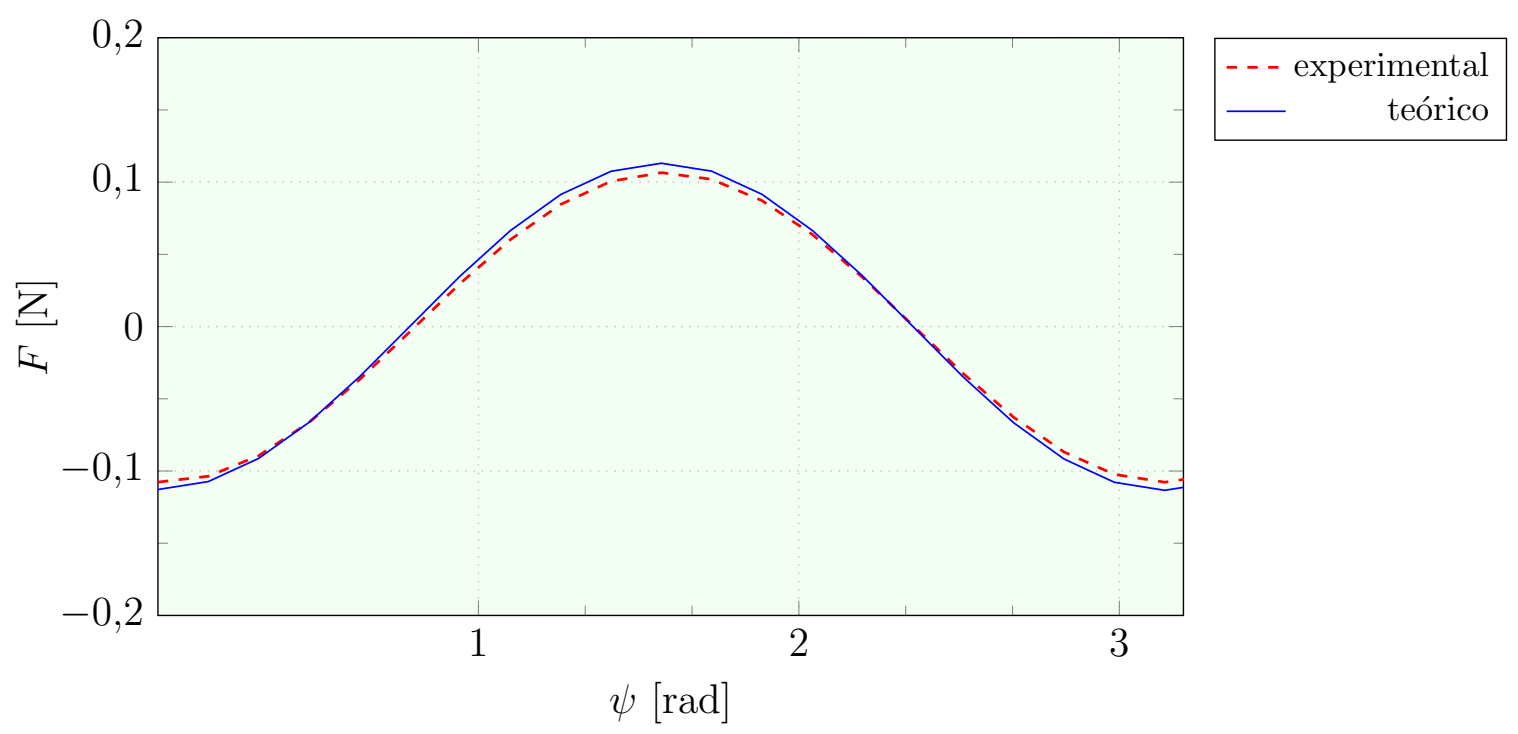

Figura 39: Comparación entre el modelo experimental de la fuerza registrada en la balanza y el modelo teórico para ángulos de paso $\theta_{0}=0,139 \mathrm{rad} \theta_{1 S}=0,104 \mathrm{rad}$. 


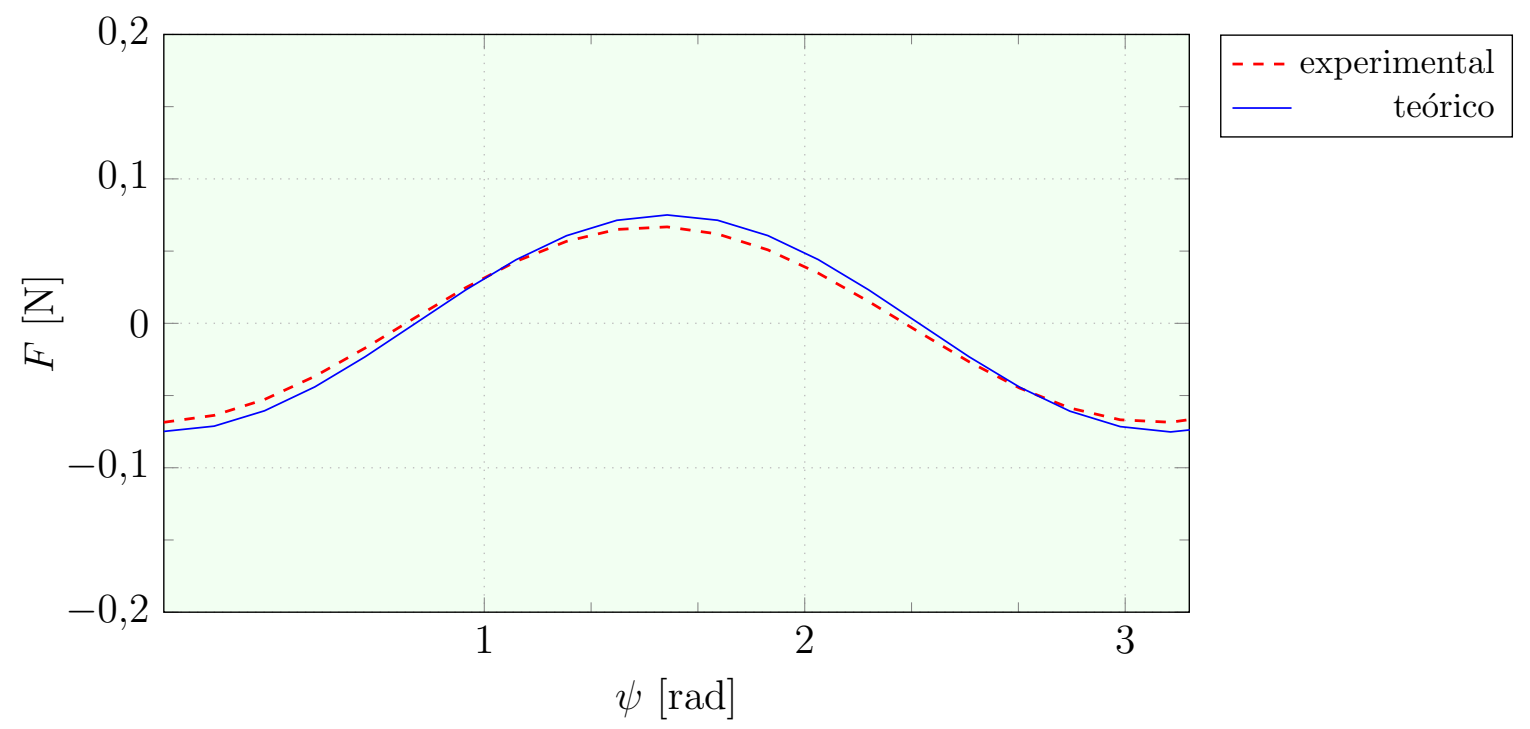

Figura 40: Comparación entre el modelo experimental de la fuerza registrada en la balanza y el modelo teórico para ángulos de paso $\theta_{0}=0,139 \mathrm{rad} \theta_{1 S}=0,069 \mathrm{rad}$.

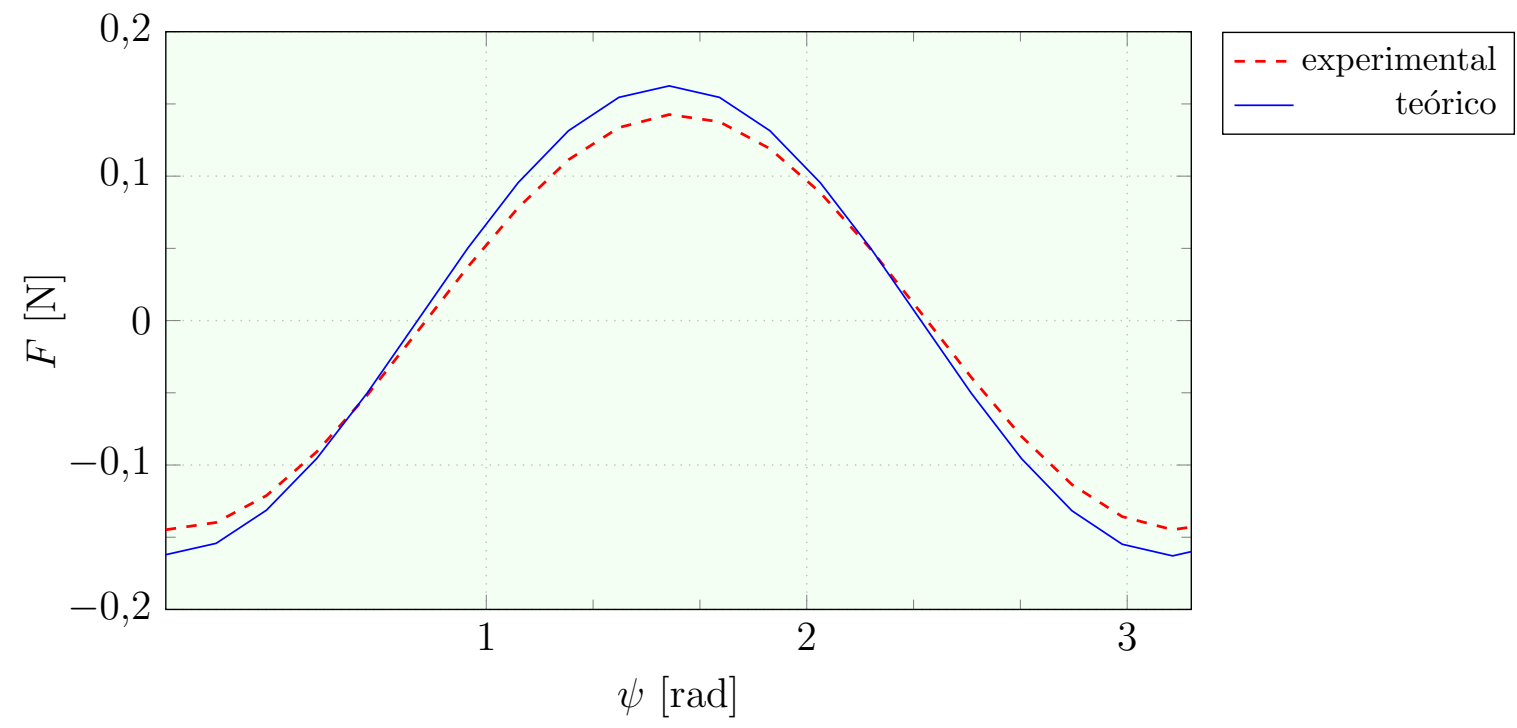

Figura 41: Comparación entre el modelo experimental de la fuerza registrada en la balanza y el modelo teórico para ángulos de paso $\theta_{0}=0,104 \mathrm{rad} \theta_{1 S}=0,139 \mathrm{rad}$. 


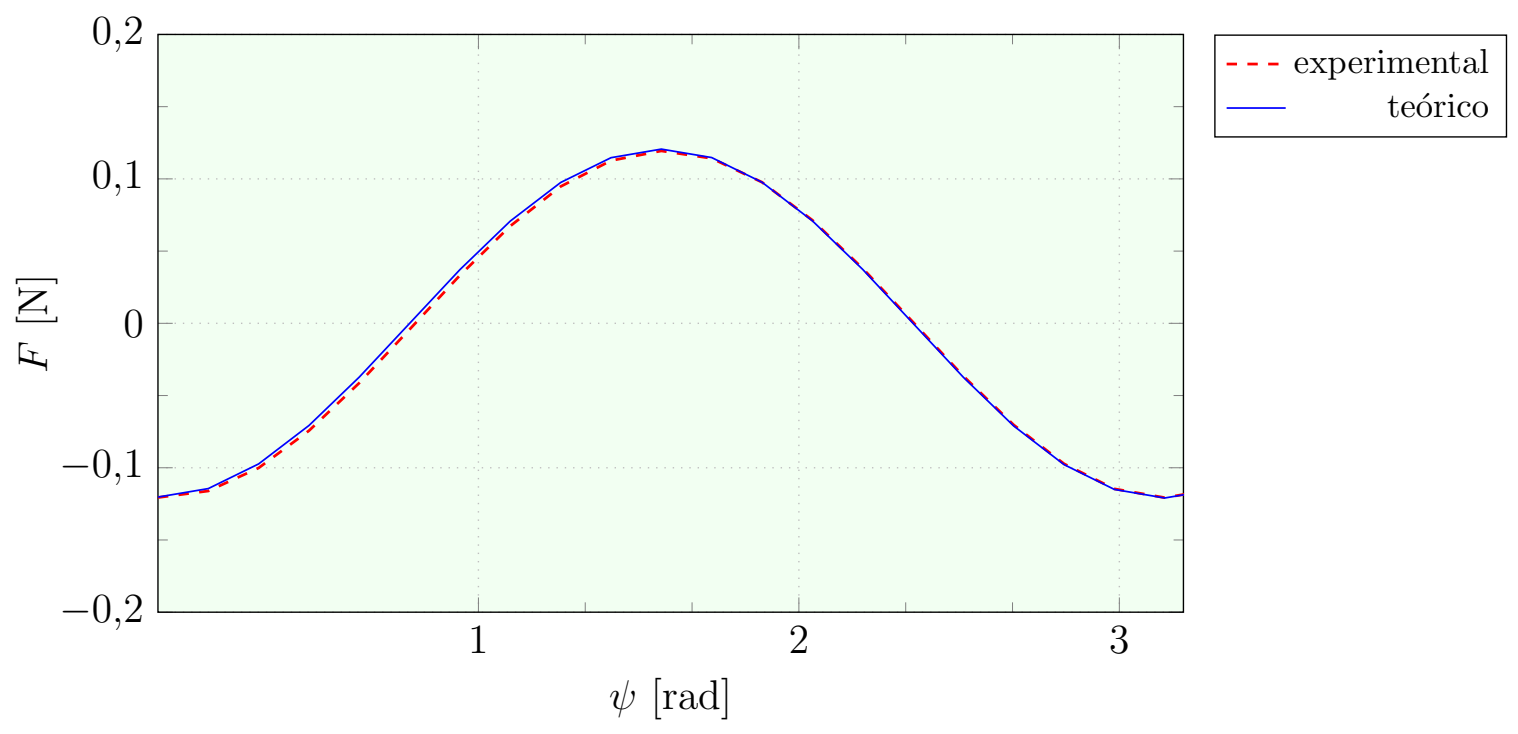

Figura 42: Comparación entre el modelo experimental de la fuerza registrada en la balanza y el modelo teórico para ángulos de paso $\theta_{0}=0,104 \mathrm{rad} \theta_{1 S}=0,104 \mathrm{rad}$.

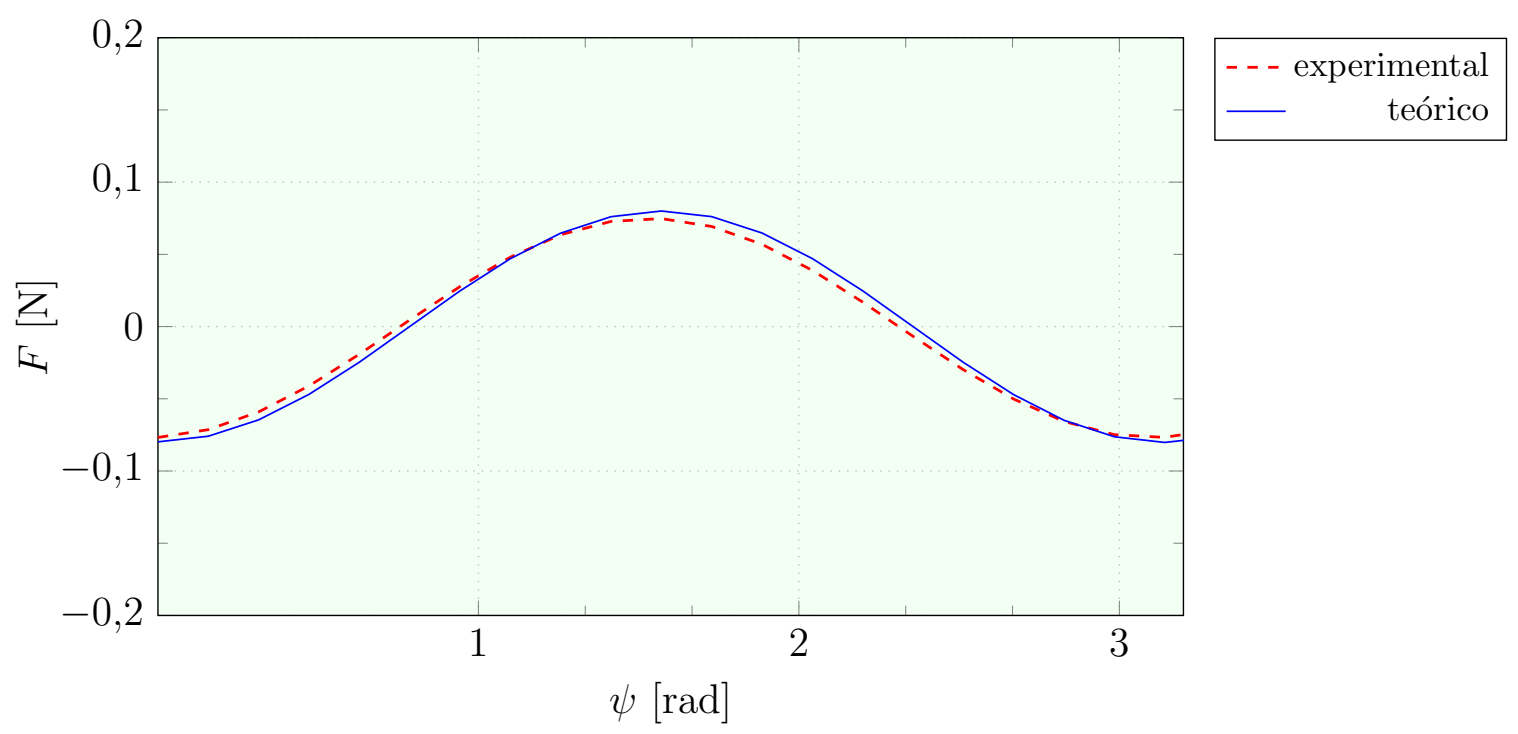

Figura 43: Comparación entre el modelo experimental de la fuerza registrada en la balanza y el modelo teórico para ángulos de paso $\theta_{0}=0,104 \mathrm{rad} \theta_{1 S}=0,069 \mathrm{rad}$. 
peso relativo que cada variable tiene en el resultado final (incertidumbre tipo B). Las expresiones a partir de la cual se estimaron estos resultados son expresiones simplificadas de la fuerza y la velocidad angular experimental, ya que las expresiones completas involucran cientos de términos que hacen impracticables los cálculos.

La ecuación (91) es la expresión que permite identificar que parámetros intervienen para la estimación de las incertidumbres en la determinación de la fuerza en la balanza. En ella se observa que, además de las longitudes características de la bancada $\left(l_{O}\right.$ y $\left.l_{C_{1}}\right)$, intervienen las expresiones de fuerzas y momentos que absorbe la balanza. Como se menciona antes, estas expresiones resultan extensas y complejas para servir al tratamiento de las incertidumbres, sin embargo se analizan expresiones sencillas de modo de obtener cotas superiores para esta incertidumbre. Estas expresiones son las que se obtienen a partir del desarrollo en series de Taylor, reteniendo términos de primer orden para los parámetros de control y de segundo orden para la relación de velocidades $\mu_{z_{A}}$ (ver apartado 3.11.1):

$$
\begin{gathered}
F_{S, y_{A}}^{t, O}=-\rho S \Omega(t)^{2} X_{P} R_{b}^{2} \mu_{z_{A}} \cos \psi C_{L \alpha}\left(\theta_{1 C} \cos \psi+\theta_{1 S} \sin \psi\right) \\
M_{x_{A}}^{a, O}=\rho S \Omega(t)^{2} X_{P} R_{b}^{3} C_{L \alpha}\left[\theta_{1 S} \sin ^{2} \psi\left(X_{P}^{2}+\frac{\mu_{z_{A}}}{2}\right)+\theta_{1 C} \sin \psi \cos \psi\left(X_{P}^{2}+\frac{\mu_{z_{A}}}{2}\right)\right. \\
+\frac{\mu_{z_{A}} \cos \psi C_{L \alpha}}{R_{b}}\left(\theta_{1 C} \cos \psi+\theta_{1 S} \sin \psi\right) l_{O A}
\end{gathered}
$$

Asignando estas expresiones en la ecuación (91) es posible acotar la incertidumbre en las mediciones de la fuerza en la balanza mediante el uso de la siguiente expresión:

$$
U_{F_{y_{A}}}^{2}=\sum_{i=1}^{n}\left(\frac{\partial F}{\partial x_{i}}\right)^{2} \cdot u_{x_{i}}^{2}
$$

donde $n$ es el número de magnitudes medidas y $u_{x_{i}}$ la incertidumbre asociada a cada una de las magnitudes medidas. Para la incertidumbre de la velocidad angular $\Omega$ se utiliza la del instrumento, ya que se realiza la medición en forma directa.

De este modo, resulta el siguiente cuadro de incertidumbre combinada:

\begin{tabular}{cccc}
\hline \hline$\theta_{1 S}[\mathrm{rad}]$ & 0,034 & 0,069 & 0,104 \\
$U_{F_{y_{A}}^{\exp }[\mathrm{N}]}\left[1 \cdot 10^{-3}\right.$ & $2 \cdot 10^{-3}$ & $5 \cdot 10^{-3}$ \\
$U_{\Omega_{e x p}}[\mathrm{rad} / \mathrm{s}]$ & 0,1 & 0,1 & 0,1 \\
\hline
\end{tabular}

Cuadro 12: Incertidumbre combinada 
Dentro de estas incertidumbres se encuentran contenidas las de origen experimental, como por ejemplo defectos en el balance de las masas rotantes o de instrumentación.

\subsection{Conclusiones del capítulo}

Se diseñó y construyó un banco de ensayos para el estudio del efecto de las variaciones cíclicas del ángulo de paso de las palas sobre los parámetros velocidad angular $\Omega$ y fuerza lateral $F$. La configuración experimental adoptada resultó siendo aquella para la cual las vibraciones mecánicas lograron reducirse. La fuerza lateral generada por el cambio de paso cíclico fue registrada unidireccionalmente (dirección $\left.x_{A}\right)$.

Se estudió experimentalmente el comportamiento de un pararrotor ante variaciones cíclicas en el ángulo de paso de las palas. El momento sobre el cuerpo del pararrotor es un fenómeno que evoluciona a lo largo de una rotación sobre su propio eje.

Se observa que la velocidad angular disminuye con el aumento del ángulo de paso cíclico. Este efecto no es representado adecuadamente mediante el modelo teórico, por lo que a partir de aquí se desprende una línea de trabajo en la que, mediante la mejora de la instrumentación a tal fin, pueda registrarse con detalle la evolución de la velocidad angular a lo largo de una vuelta.

Se confirma que la variación cíclica del ángulo de paso de las palas permite controlar el momento aerodinámico que generan las palas alrededor del centro de masas del cuerpo del pararrotor. Esta observación se desprende del hecho de que la fuerza lateral registrada por la balanza varía a medida que lo hace el ángulo de paso (tanto cíclico como colectivo).

El modelo teórico desarrollado, adaptado a la realización del ensayo en el túnel de viento, reproduce los resultados obtenidos en los ensayos. Se observan diferencias máximas del orden del $13 \%$ en los valores de la fuerza lateral medida por la balanza, atribuibles tanto a factores experimentales como teóricos.

Analizando el conjunto de figuras 36 a 43 , se observa que el incremento en el ángulo de paso cíclico aumenta el momento que se alcanza.

También se observa que, a lo largo de una vuelta, el momento aerodinámico respecto al punto A del pararrotor pasa dos veces por el valor máximo, debido a la contribución de las dos palas simultáneamente. 


\section{Simulaciones de trayectorias del desplazamien- to de un decelerador aerodinámico a través de las variaciones cíclicas del paso de las palas}

\section{$5.1 \quad$ Introducción}

El modelo dinámico desarrollado en el capitulo 3 describe el comportamiento en vuelo de un pararrotor. Las distintas configuraciones que tome el vector de control, definido por el ángulo de paso colectivo y los ángulos de paso cíclico lateral y longitudinal, determinarán el tipo de trayectoria que describa el dispositivo en un régimen de descenso en autorrotación. En este capítulo de recuperan los conceptos elaborados en el capítulo 3 para estudiar las trayectorias que puede tomar un pararrotor diseñado bajo los conceptos desarrollados en el documento. Partiendo del modelo dinámico no lineal y la representación matemática obtenida para las fuerzas y los momentos, se analizan hipótesis simplificativas para la obtención de un vector de control que garantice valores cercanos al equilibrio para las variables de estado. Las ecuaciones simplificadas son resueltas implementando un código numérico, que entrega como solución el vector de control necesario para el cumplimiento de las condiciones de operación pre establecidas.

La segunda parte del estudio, se realiza a partir de la implementación de otro código de simulación numérica que reproduce el modelo completo de la dinámica del pararrotor. El vector de control, obtenido para un modelo simplificado del pararrotor, se utiliza para estudiar la operación del sistema y la implementación de algoritmos de control elementales sobre el modelo completo, en vistas de generar un aporte al control y el guiado de los pararrotores.

\subsection{Modelo matemático}

El comportamiento de un pararrotor en vuelo libre puede modelarse mediante el uso de las ecuaciones Newton-Euler aplicadas a un sistema de cuerpos rígidos. Las características constructivas de este tipo de dispositivos permiten supo- 
ner en primera instancia esta conducta, ya que los grados de libertad asociados a la deformación elástica de las palas (batimiento, arrastre y paso) pueden despreciarse respecto a los que involucran las ecuaciones de Newton-Euler. El modelo matemático se desarrolló en el capítulo 3 partiendo de las siguientes ecuaciones:

$$
\begin{gathered}
\mathbf{F}^{t}+\mathbf{F}_{H}^{g}+\mathbf{F}_{H}^{a}+\mathbf{F}_{H}^{i, G}=0 \\
\mathbf{M}^{t, G}+\mathbf{M}_{H}^{a, G}+\mathbf{M}_{H}^{i, G}=0
\end{gathered}
$$

Analizando cada uno de los términos de (97a) y (97b) se obtienen las expresiones representativas correspondientes.

Es posible establecer una relación entre la razón de cambio temporal del vector de estado del pararrotor y el propio vector de estado. Esta relación es no lineal, ya que existen términos de alto orden que no pueden ser despreciados. Esta relación queda establecida analíticamente como:

$$
\mathbf{M} \frac{d \mathbf{x}(t)}{d t}=\mathbf{f}
$$

La resolución de esta ecuación diferencial, partiendo de condiciones iniciales $\mathbf{x}_{0}=\mathbf{x}(0)$ adecuadas permite conocer la evolución temporal del vector de estado $\mathbf{x}(t)$ en función del vector de control $\mathbf{u}_{C}(t)$. Particularmente, la integración del vector velocidad define el vector posición del centro de masas $\mathbf{r}^{G}(t)$, y por lo tanto la trayectoria del centro de masas del pararrotor.

\subsection{Vuelo equilibrado del pararrotor}

Se define el vuelo equilibrado como aquel en el que la velocidad del centro de masas del pararrotor expresada en ejes tierra, $\mathbf{V}^{G}$, es constante, y no hay cambio de actitud $\left(\omega_{x}=\omega_{y}=0\right)$. Para una condición de paso cíclico no nulo, aunque los ángulos de control $\theta_{0}, \theta_{1 S}$ y $\theta_{1 C}$ sean constantes, no es posible, en general, encontrar soluciones del sistema (98) que satisfagan esta condición. Es decir, no existe ninguna configuración constante del vector de control $\mathbf{u}_{C}$ para que las primeras ocho componentes del vector de estado $\mathbf{x}_{12}$ permanezcan invariantes en el tiempo. Particularmente las velocidades angulares $\omega_{x}$ y $\omega_{y}$ no alcanzan esta condición de equilibrio debido al efecto de las fuerzas y momentos aerodinámicos, que varían con el ángulo de azimut. Es decir, para cada posición azimutal del pararrotor, la magnitud de las fuerzas y momentos aerodinámicos es distinta, y, en consecuencia, también la velocidad angular.

En la figura 44 se presenta un esquema de las fuerzas que actúan sobre un modelo básico del pararrotor. Las acciones de las palas, representadas por 
los vectores $\mathbf{F}^{t}$ y $\mathbf{M}^{t, A}$, las del propio cilindro, representadas por $\mathbf{F}_{H}^{a}$ y $\mathbf{M}_{H}^{a, F}$ y el peso del pararrotor forman el conjunto de fuerzas y momentos que determinan el comportamiento dinámico del mismo.

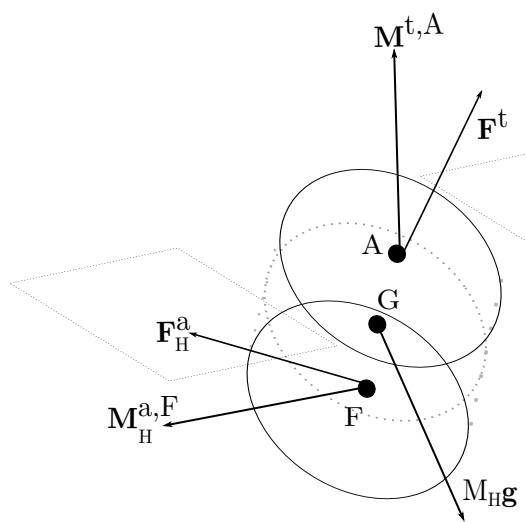

Figura 44: Esquema de fuerzas sobre el pararrotor en vuelo libre

Las fuerzas y momentos que tienen lugar sobre el pararrotor varían temporalmente en el período que requieren las palas para describir una revolución y, en general, son rápidas respecto a la dinámica del cuerpo del pararrotor. Bajo esta idea conceptual es que se realizan simplificaciones en las fuerzas y momentos para modelizarlas.

\subsubsection{Modelado de las acciones transmitidas al pararrotor}

Las acciones dinámicas sobre los distintos elementos que constituyen el pararrotor se representan mediante una fuerza y un momento aplicado sobre un punto de referencia (punto A).

De este modo se tiene que las palas transmiten instantáneamente una fuerza $\mathbf{F}_{b}^{t}$ y un momento respecto al punto de acoplamiento $E_{b}, \mathbf{M}^{t, E_{b}}$, expresiones que contienen tanto componentes aerodinámicas como gravitatorias e inerciales. Estas acciones varían temporalmente en el período que requiere la pala para describir una revolución, y, como se menciona anteriormente, pueden suponerse extremadamente rápidas respecto al tiempo que requieren las variables de estado para modificarse de manera significativa. Por ejemplo, si se supone un régimen de operación de $\omega_{z}=80 \mathrm{rad} / \mathrm{s}$ (que representa un valor típico alcanzado en los ensayos), el tiempo en el que realiza una vuelta es $t=0,01 \mathrm{~s}$, que, comparado con el tiempo de realización de una maniobra, es despreciable.

No obstante, los valores medios de estas fuerzas son los que modifican el estado del sistema. Estos promedios pueden calcularse como: 


$$
\begin{gathered}
\overline{\mathbf{F}}_{b}^{t}=\frac{1}{2 \pi} \int_{0}^{2 \pi} \mathbf{F}_{b}^{t} d \psi \\
\overline{\mathbf{M}}_{b}^{t, A}=\frac{1}{2 \pi} \int_{0}^{2 \pi}\left(\mathbf{M}_{b}^{t, E}+\mathbf{A} \mathbf{E}_{b} \wedge \mathbf{F}_{b}^{t}\right) d \psi
\end{gathered}
$$

Para el cuerpo del pararrotor, se considera que las fuerzas y momentos aerodinámicos que introduce son despreciables respecto a aquellas que introducen las palas, por lo que, en una primera aproximación, se considera nulo el aporte de las mismas. Puede justificarse esta afirmación utilizando valores de coeficientes de resistencia típicos para cilindros, y determinando el orden de magnitud de la fuerza de resistencia que se genera sobre el cuerpo. Tomando valores de referencia para $C_{D}$ [33], se puede cuantificar la fuerza de resistencia como $F=\frac{1}{2} \rho \mathbf{U}_{W}^{2} S_{H} C_{D_{H}} \approx 0,02$ $\mathrm{N}$, que resulta dos órdenes de magnitud menor que la fuerza que generan las palas.

Estos modelos para las acciones transmitidas permiten determinar los valores que toman las variables de estado para distintas condiciones iniciales seleccionadas del vector de estado.

\subsubsection{Forma canónica de la ecuación de equilibrio}

El modelo de fuerzas y momentos promediados azimutalmente permite plantear que existe un estado $\mathbf{x}_{e}$ en el que tanto la velocidad $\mathbf{V}^{G}(t)$ como la velocidad angular $\boldsymbol{\omega}(t)$ (a excepción de la variable $x_{9}=\psi$ ) se mantienen constantes. Así, el modelo desarrollado en el capítulo 3 se convierte en

$$
\begin{gathered}
\overline{\mathbf{F}}^{t}+\overline{\mathbf{F}}_{H}^{a}+M_{H} \mathbf{g}=0 \\
\overline{\mathbf{M}}^{t, A}+\overline{\mathbf{M}}_{H}^{a, G}=0
\end{gathered}
$$

Como se menciona, a excepción de $\psi$, el resto de las variables de estado permanecen invariantes en el tiempo. A partir de esta definición se pueden establecer dos condiciones, a saber:

- La velocidad angular del pararrotor en ejes A queda definida como $\left.\boldsymbol{\omega}\right|_{A}=$ $\left[0,0, \omega_{z}\right]^{\prime}$, que conduce, en vistas de la condición de ligadura cinemática, a establecer que $\Theta$ y $\Phi$ son constantes, no así $\psi$.

- Al ser $\Theta$ y $\Phi$ constantes, junto con la velocidad del centro de masas en ejes A, implica que la velocidad del centro de masas en ejes tierra también sea constante. Esta condición se deduce a partir de que, siendo las matrices de 
transformación $\left[\mathbf{T}_{T 1 T}\right]$ y $\left[\mathbf{T}_{A T 1}\right]$ constantes, se puede definir la posición del centro de masas del pararrotor integrando la ecuación (17):

$$
\mathbf{r}^{G}=\mathbf{r}_{0}^{G}+\left[u_{T}, v_{T}, y_{T}\right]^{\prime} t
$$

Esta condición de equilibrio permite obtener 6 ecuaciones con 9 parámetros, que corresponden a 6 componentes del vector de estado reducido en equilibrio $\left(\mathbf{x}_{r, e}=\left[u, v, w, \omega_{z}, \Theta, \Phi\right]\right)$ y a las 3 del vector de control $\left(\mathbf{u}_{C, e}\right)$. Por lo tanto, prefijando tres parámetros es posible determinar el valor del resto.

Las ecuaciones correspondientes a la utilización de fuerzas y momentos promediados en la vuelta quedan planteadas en el formato canónico tal como se muestran a continuación:

$$
\frac{1}{2 \pi} \int_{0}^{2 \pi}\left\{\mathbf{M}_{6 \times 9}\left[\begin{array}{c}
\mathbf{0}_{8} \\
\omega_{z}
\end{array}\right]-\mathbf{f}_{6 \times 1}\right\} d \psi=\mathbf{0}
$$

El problema se completa incorporando 5 nuevas variables, junto a las correspondientes nuevas ecuaciones, a saber:

- Las componentes de $\mathbf{V}^{G}$ en el sistema de referencia tierra $\left.\mathbf{V}^{G}\right|_{T}=\left[u_{T}, v_{T}, w_{t}\right]$,

- El ángulo entre el eje $x_{T}$ y la velocidad de desplazamiento $\left(\mathbf{V}^{G}\right) \beta_{T}$ (denominado ángulo de dirección horizontal),

- El módulo de la velocidad $\mathbf{V}^{G}, V_{H}$.

Las ecuaciones que completan el sistema son :

- La transformación de $\mathbf{V}^{G}$ de ejes cuerpo a ejes tierra,

$$
\left[\begin{array}{c}
u_{T} \\
v_{T} \\
w_{T}
\end{array}\right]=\left[\mathbf{T}_{T T 1}(\Theta)\right]\left[\mathbf{T}_{T 1 A}(\Phi)\right]\left[\begin{array}{c}
u \\
v \\
w
\end{array}\right]
$$

- La definición de $V_{H}$ y $\beta_{T}$ en términos de $u_{T}$ y $v_{T}$,

$$
\begin{gathered}
\beta_{T}=\arctan \frac{v_{T}}{u_{T}} \\
V_{H}=\sqrt{u_{T}^{2}+v_{T}^{2}}
\end{gathered}
$$

El vector de estado del sistema ampliado a 11 componentes, denominado $\mathbf{y}$, se muestra a continuación: 


$$
\mathbf{y}=\left[\begin{array}{c}
u \\
v \\
w \\
\omega_{z} \\
\Theta \\
\Phi \\
u_{T} \\
v_{T} \\
w_{T} \\
\beta_{T} \\
V_{H}
\end{array}\right]
$$

La figura siguiente muestra las magnitudes definidas:

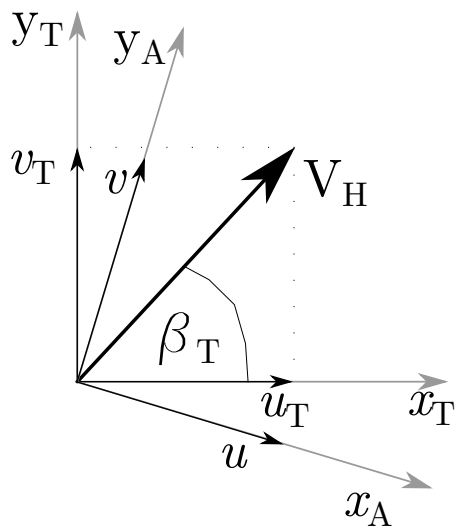

Figura 45: Diagrama de velocidades y ángulos auxiliares

Para conocer el estado de equilibrio, junto con los parámetros de control correspondientes, se deben resolver de manera conjunta las ecuaciones (103) a (106), que sintéticamente se escribe:

$$
\mathbf{F}_{11}\left(\mathbf{y}_{e}, \mathbf{u}_{C_{e, 3}}\right)=\mathbf{0}
$$

\subsubsection{Características de la solución del equilibrado}

Tal como se menciona en el apartado anterior, la definición a priori de tres parámetros del vector de estado permite obtener el resto de los parámetros para el caso de un estado de operación cercano al equilibrio del pararrotor. Claro está que la solución a la que se arriba es la que corresponde al caso simplificado de considerar las fuerzas y momentos de las palas promediados a lo largo de una vuelta, por lo que 
deberá analizarse el desempeño de la solución del sistema de equilibrio en el modelo no lineal desarrollado en el capítulo 3.

A modo ilustrativo se analiza la solución de un caso particular del equilibrio. La solución del sistema que se presenta es la que corresponde a una condición de vuelo con ángulo de dirección horizontal $\left(\beta_{T}\right)$ y velocidad de descenso $\left(w_{T}\right)$ constantes, para diferentes velocidades de desplazamiento $\left(V_{H}\right)$. Estos son los tres parámetros que se definen para la resolución del sistema (108) . El modelo de pararrotor que se utiliza mantiene las mismas características que las del resto del documento.

La configuración del vector de estado prefijado para los distintos casos analizados se resume en el cuadro 13 :

\begin{tabular}{cc}
\hline \hline Parámetro & Valor \\
\hline$\beta_{T}$ & 0 rad \\
$w_{T}$ & 8 a $10 \mathrm{~m} / \mathrm{s}$ \\
$V_{H}$ & 0 a $5 \mathrm{~m} / \mathrm{s}$ \\
\hline \hline
\end{tabular}

Cuadro 13: Elementos prefijados del vector de estado

La implementación numérica del sistema (108) permite resolverlo y obtener el vector de estado y el de control en el equilibrio. Los resultados obtenidos se muestran en el conjunto de figuras 46.

Las figuras 46 presentan la solución del sistema equilibrado para los parámetros del vector de control $\left(\theta_{0}, \theta_{1 S}\right.$ y $\left.\theta_{1 C}\right)$, la velocidad angular $\left(\omega_{z}\right)$ y los ángulos de cabeceo $(\Theta)$ y balanceo $(\Phi)$ en función de la velocidad de desplazamiento horizontal del pararrotor $\left(V_{H}\right)$, parametrizados con la velocidad de descenso $\left(w_{T}\right)$.

Si se analiza la evolución del ángulo de cabeceo, $\Theta$, puede decirse que para desplazarse a velocidades más rápidas el pararrotor debe inclinarse respecto a la vertical. Esto es razonable, ya que la inclinación del pararrotor genera una componente de fuerza mayor en la dirección de desplazamiento. El ángulo de balanceo, $\Phi$, es cada vez mayor a medida que aumenta la velocidad de desplazamiento del pararrotor.

En las figuras 46c y 46d se muestran los ángulos de paso cíclico lateral y longitudinal, que resultan los necesarios para establecer la condición de vuelo definida. En ellas se observa que la magnitud del ángulo de paso cíclico lateral $\theta_{1 C}$ es un orden menor que la del ángulo de paso cíclico longitudinal $\theta_{1 S}$. Se puede decir que la velocidad de traslación para $\beta_{T}=0$ es controlada principalmente por el ángulo $\theta_{1 S}$. También puede observarse el comportamiento no lineal que presentan los ángulos de paso cíclico, indicando que el cambio en la velocidad horizontal $V_{H}$ requiere 


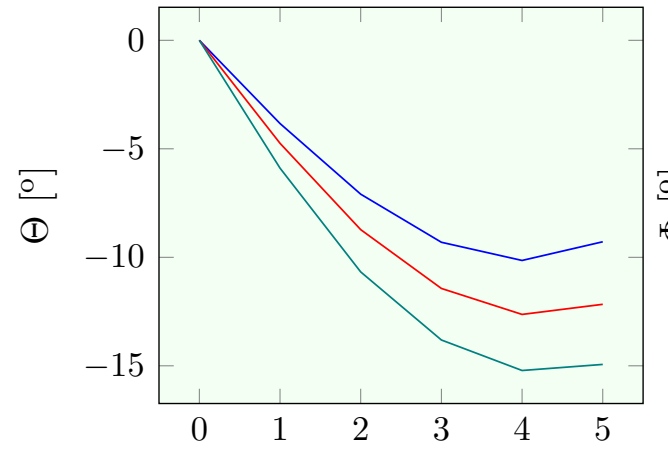

(a) $V_{H}[\mathrm{~m} / \mathrm{s}]$

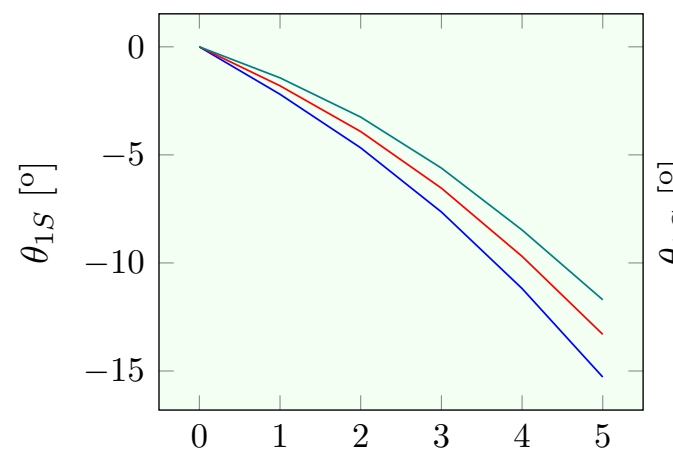

(c) $V_{H}[\mathrm{~m} / \mathrm{s}]$

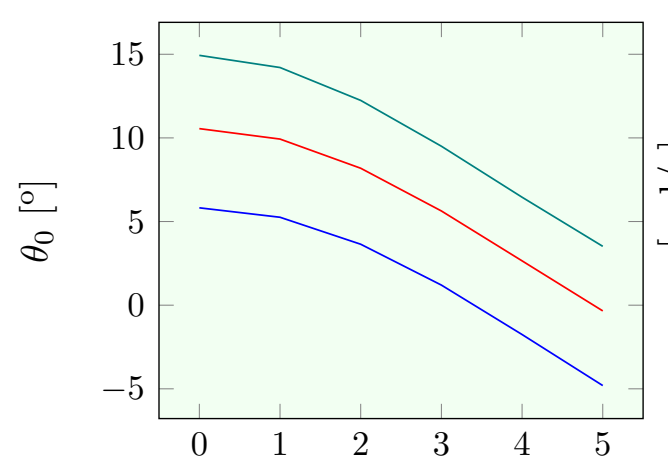

(e) $V_{H}[\mathrm{~m} / \mathrm{s}]$

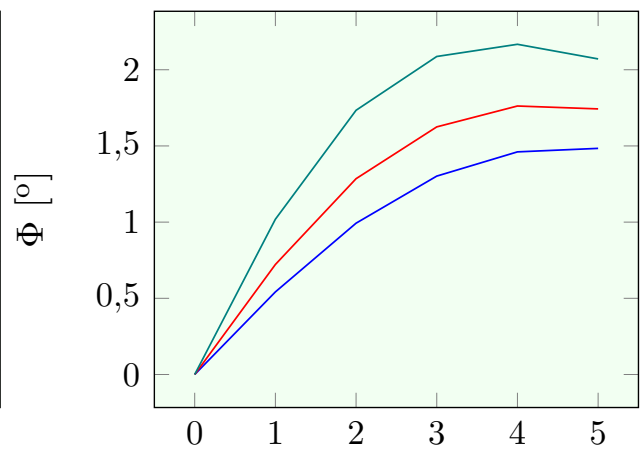

(b) $V_{H}[\mathrm{~m} / \mathrm{s}]$

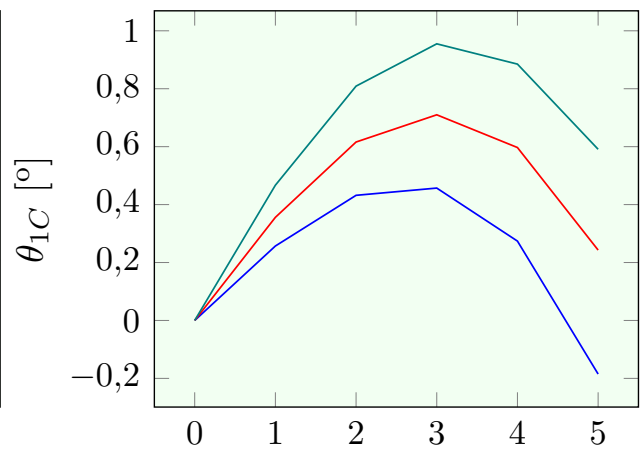

(d) $V_{H}[\mathrm{~m} / \mathrm{s}]$

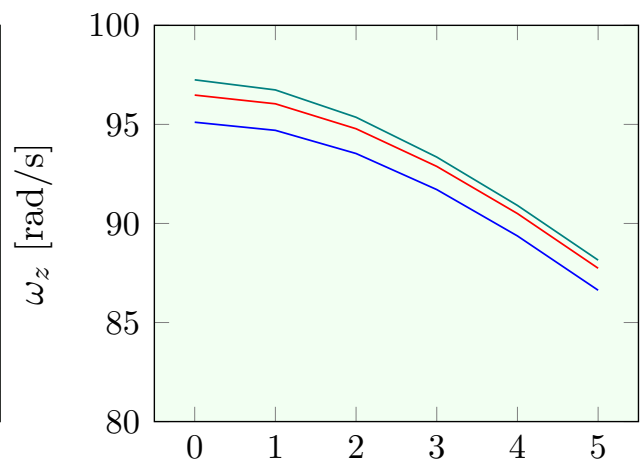

(f) $V_{H}[\mathrm{~m} / \mathrm{s}]$

$-w_{T}=8 \mathrm{~m} / \mathrm{s}-w_{T}=9 \mathrm{~m} / \mathrm{s}-w_{T}=10 \mathrm{~m} / \mathrm{s}$

Figura 46: Soluciones del sistema equilibrado 
incrementos distintos en el ángulo de paso. En el caso del ángulo de paso cíclico longitudinal $\left(\theta_{1 S}\right)$, se observa que es más sensible (tiene pendiente más pronunciada) a mayores velocidades de desplazamiento. El ángulo de paso cíclico lateral $\left(\theta_{1 C}\right)$ muestra dos regiones de mayor sensibilidad $\left(V_{H}<2 \mathrm{~m} / \mathrm{s}\right.$ y $\left.V_{H}>3 \mathrm{~m} / \mathrm{s}\right)$ y un rango prácticamente constante en los valores intermedios $\left(2 \mathrm{~m} / \mathrm{s}<V_{H}<3 \mathrm{~m} / \mathrm{s}\right)$.

Respecto al ángulo de paso colectivo (46e) se observa que disminuye conforme aumenta la velocidad de desplazamiento. También disminuye si se buscan velocidades de descenso $w_{T}$ menores. Se identifica una región de pendiente variable (para $V_{H}<2 \mathrm{~m} / \mathrm{s}$ ) y luego una variación lineal del mismo parámetro en función de la velocidad de desplazamiento horizontal $V_{H}$.

De la figura que representa la velocidad angular $\omega_{z}$ (figura 46f) se observa que la misma disminuye conforme aumenta la velocidad de traslación. El valor máximo se da para descensos puramente verticales $\left(V_{H}=0\right)$. Este comportamiento es analizado de forma global junto al diagnóstico de la solución dinámica para el vector de control seleccionado que se realiza en la siguiente sección.

Por ejemplo, si se toma el caso correspondiente a un desplazamiento puro en la dirección $x_{T}$, una velocidad de descenso $w_{T}=8 \mathrm{~m} / \mathrm{s}$ y una velocidad de desplazamiento horizontal $V_{H}=3 \mathrm{~m} / \mathrm{s}$, se tiene la siguiente solución:

$$
\mathbf{y}_{e}=\left[\begin{array}{c}
u \\
v \\
w \\
\omega_{z} \\
\Theta \\
\Phi \\
u_{T} \\
v_{T} \\
w_{T} \\
\beta_{T} \\
V_{H}
\end{array}\right]=\left[\begin{array}{c}
4,25 \mathrm{~m} / \mathrm{s} \\
0,16 \mathrm{~m} / \mathrm{s} \\
7,40 \mathrm{~m} / \mathrm{s} \\
91,7 \mathrm{rad} / \mathrm{s} \\
-0,16 \mathrm{rad} \\
0,02 \mathrm{rad} \\
3 \mathrm{~m} / \mathrm{s} \\
0 \mathrm{~m} / \mathrm{s} \\
8 \mathrm{~m} / \mathrm{s} \\
0 \mathrm{rad} \\
3 \mathrm{~m} / \mathrm{s}
\end{array}\right], \mathbf{u}_{C e}=\left[\begin{array}{c}
\theta_{0} \\
\theta_{1 C} \\
\theta_{1 S}
\end{array}\right]=\left[\begin{array}{c}
0,02 \mathrm{rad} \\
0,008 \mathrm{rad} \\
-0,13 \mathrm{rad}
\end{array}\right]
$$

Esta condición de equilibrio es la que se utiliza en el siguiente apartado como condición inicial para la resolución del problema dinámico completo. Para esta condición de equilibrio se tiene la siguiente evolución del ángulo de paso y del ángulo de ataque a lo largo de una vuelta: 


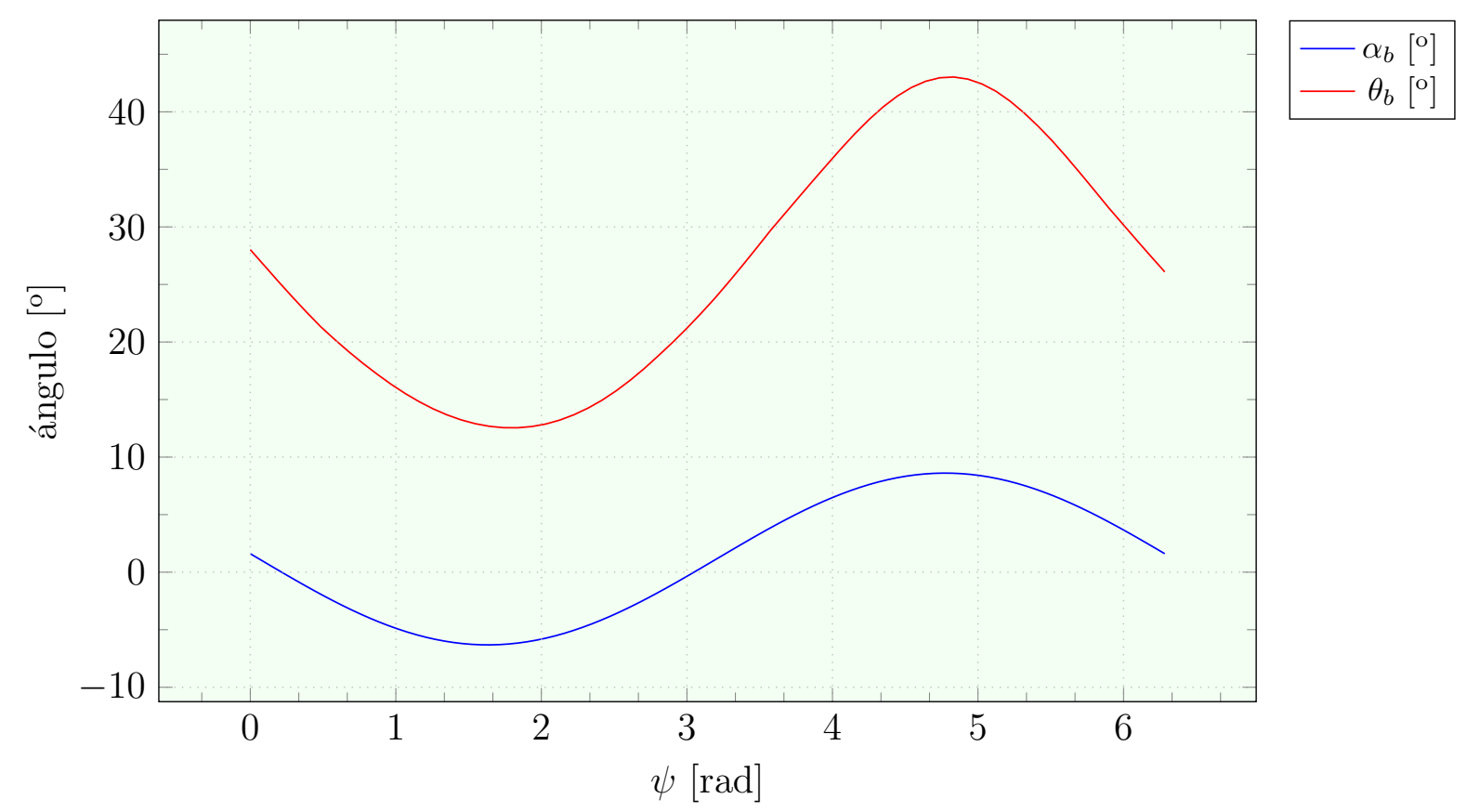

Figura 47: Ángulo de paso y de ataque de la pala del sistema equilibrado

En esta figura puede observarse como el ángulo de paso de la pala $\left(\theta_{b}\right)$ se encuentra en fase con el ángulo de ataque $\left(\alpha_{b}\right)$. En la primera media vuelta $(\psi \in$ $[0, \pi])$ la diferencia entre ambas magnitudes es menor que en la segunda media vuelta $(\psi \in[\pi, 2 \pi])$, posiblemente debido a la diferencia en la configuración aerodinámica que tiene la pala en el avance o el retroceso respecto a la dirección de desplazamiento ( $x_{T}$ en el caso particular bajo análisis).

\subsubsection{Solución del problema dinámico completo a partir de leyes de con- trol obtenidas en la solución del equilibrio}

Las configuraciones de control estudiadas en el apartado anterior son aquellas que se obtienen a partir de suponer el promedio azimutal de las acciones aerodinámicas que introducen las palas. Este factor representa una simplificación en el desarrollo del modelo que debe ser evaluada.

Si bien existen técnicas de optimización de trayectorias que pueden analizarse para ser implementadas en el sistema dinámico completo, queda fuera del alcance del presente estudio.

La pregunta que se pretende responder es si la ley de control constante determinada como solución del problema algebraico (108) da lugar a una solución de la dinámica completa tal que el vector de estado $\mathbf{x}_{12}$ tienda a la solución pretendida. 
No se busca determinar rigurosamente las condiciones de existencia y unicidad de la solución algebraica que determina la solución del modelo completo buscada si no ilustrar casos particulares en donde si lo sea.

El paso siguiente implica la evaluación en el sistema dinámico completo de la configuración de equilibrio obtenida, pudiendose eventualmente recalcular el vector de control con el objetivo de que el sistema no lineal reproduzca el comportamiento deseado.

El primer indicador que se puede utilizar para evaluar la bondad de la solución de equilibrio encontrada es el balance de fuerzas y momentos promediados sobre el pararrotor, que, como es natural esperar, debe ser nulo para todas sus componentes. En el siguiente cuadro se muestra la contribución de cada tipo de acción sobre el pararrotor según sus componentes:

\begin{tabular}{ccccccc}
\hline \hline Componente & $\bar{F}_{x_{A}}[\mathrm{~N}]$ & $\bar{F}_{y_{A}}[\mathrm{~N}]$ & $\bar{F}_{z_{A}}[\mathrm{~N}]$ & $\bar{M}_{x_{A}}[\mathrm{Nm}]$ & $\bar{M}_{y_{A}}[\mathrm{Nm}]$ & $\bar{M}_{z_{A}}[\mathrm{Nm}]$ \\
\hline gravitatoria & 0,6863 & 0,1272 & 2,6335 & 0 & 0 & 0 \\
inercial & $-0,0030$ & $-0,0026$ & -0.0090 & $-0,0003$ & $-0,00003$ & $-0,00007$ \\
aerodinámica & $-0,6856$ & $-0,1240$ & $-2,6338$ & $-0,0001$ & 0,0031 & $-0,00005$ \\
total & $-0,0003$ & 0,0006 & $-0,0093$ & $-0,0004$ & 0,00309 & 0 \\
\hline \hline
\end{tabular}

Cuadro 14: Fuerzas y momentos de equilibrio para $\beta_{T}=0, w_{T}=8 \mathrm{~m} / \mathrm{s}$ y $V_{H}=3 \mathrm{~m} / \mathrm{s}$

La configuración de control hallada para diversas condiciones de vuelo propuestas es utilizada en esta sección para la evaluación del desempeño del pararrotor en su modelo completo.

\subsubsection{Solución de la dinámica del pararrotor para desplazamiento la- teral con velocidades de descenso y traslación variables}

En virtud de ilustrar este comportamiento se continúa con el estudio de los casos ejemplificados en el apartado 5.3.3, donde se utilizaron las condiciones de vuelo que se mostradas en el cuadro 13. En este apartado, se particulariza el análisis a los parámetros presentados en el siguiente cuadro:

\begin{tabular}{cl}
\hline \hline Parámetro & Valor \\
\hline$\beta_{T}$ & $0 \mathrm{rad}$ \\
$w_{T}$ & $8 \mathrm{~m} / \mathrm{s}$ \\
$V_{H}$ & $0 \mathrm{a} 3 \mathrm{~m} / \mathrm{s}$ \\
\hline \hline
\end{tabular}

Cuadro 15: Elementos prefijados del vector de estado 
El vector solución presentado en (109) es el que permite obtener el conjunto de condiciones iniciales para el vector de estado y el vector de control constante para resolver el sistema dinámico completo (98). De este modo, resulta el siguiente conjunto de condiciones iniciales:

$$
\begin{gathered}
\mathbf{x}=\left[\begin{array}{c}
\mathbf{0}_{5} \\
\frac{\omega_{z, e}}{4} \\
\mathbf{0}_{5} \\
r_{G_{3, i}}
\end{array}\right] \\
\mathbf{u}_{C_{V_{H}=0}}=\left[\begin{array}{c}
0,101 \mathrm{rad} \\
0,00 \mathrm{rad} \\
0,00 \mathrm{rad}
\end{array}\right], \mathbf{u}_{C_{V_{H}=1}}=\left[\begin{array}{c}
0,091 \mathrm{rad} \\
0,004 \mathrm{rad} \\
-0,038 \mathrm{rad}
\end{array}\right], \\
\mathbf{u}_{C_{V_{H}=2}=}=\left[\begin{array}{c}
0,063 \mathrm{rad} \\
0,007 \mathrm{rad} \\
-0,081 \mathrm{rad}
\end{array}\right], \mathbf{u}_{C_{V_{H}=3}}=\left[\begin{array}{c}
0,021 \mathrm{rad} \\
0,008 \mathrm{rad} \\
-0,133 \mathrm{rad}
\end{array}\right]
\end{gathered}
$$

En donde se indica que la velocidad inicial del centro de masas es nula, como así también las componentes $\omega_{x} \mathrm{y} \omega_{y}$ de la velocidad angular $\left.\boldsymbol{\omega}\right|_{A}$. Los ángulos de Euler son nulos y la posición inicial del centro de masas es $\mathbf{r}_{i}^{G}=-1000 \mathbf{k}_{T}$. El subíndice en el vector de control $\mathbf{u}_{C}$ indica la velocidad de desplazamiento horizontal para la cual corresponde.

Las figuras 48 muestran el estado dinámico del pararrotor para la ley de control obtenida a partir de la solución del sistema (108). Representa la solución del sistema (33) para las condiciones de equilibrio halladas durante un intervalo de tiempo $\left(t_{s}\right)$ de 40 segundos.

Se observa que el pararrotor describe la trayectoria prevista (figura 48a), con un desplazamiento lateral en la dirección $y_{T}$ menor al $2 \%$ respecto al que tiene según la dirección $x_{T}$. Es convenienta aclarar, para la facilitación en la interpretación de la figura 48a, que la escala del eje vertical es distinta a la del eje horizontal. El alcance lateral según la dirección $x_{T}$, dado por la componente $r_{G_{1}}$ del vector de posición del centro de masas, es prácticamente igual al producto $V_{H} \cdot t_{s}$. Por ejemplo, si la velocidad de desplazamiento es $V_{H}=3 \mathrm{~m} / \mathrm{s}$, la distancia horizontal alcanzada en vuelo de $t_{s}=40 \mathrm{~s}$ es $x_{T} \approx 120 \mathrm{~m}$. Esto significa que, según la velocidad de desplazamiento horizontal deseada, se puede alcanzar la posición final buscada.

Como es lógico, a medida que se configura el vector de control para incrementar la velocidad de desplazamiento $V_{H}$ se obtienen mayores alcances. En la figura 48b se observa que para el mismo tiempo de caída se obtienen desplazamientos de hasta $120 \mathrm{~m}$. 


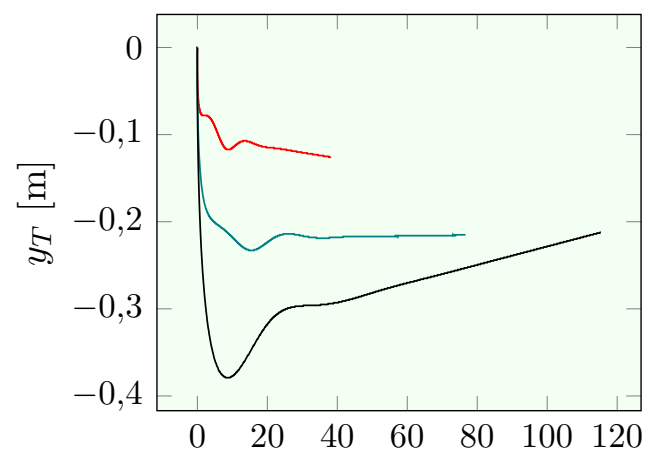

(a) $x_{T}[\mathrm{~m}]$

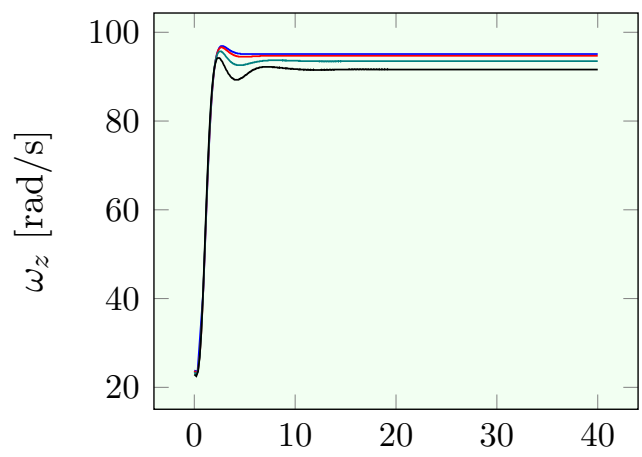

(c) $t[\mathrm{~s}]$

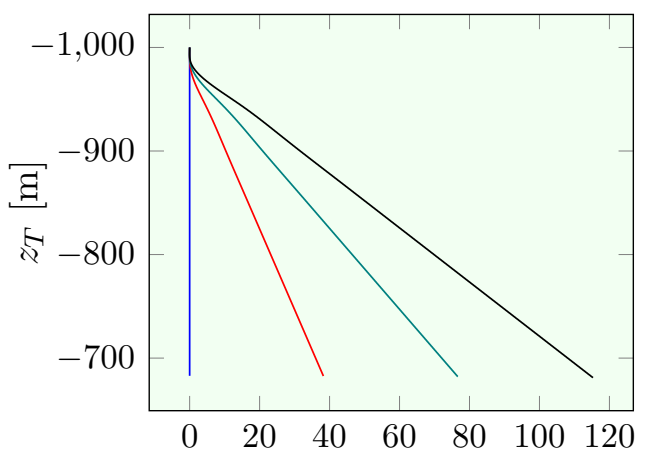

(b) $x_{T}[\mathrm{~m}]$

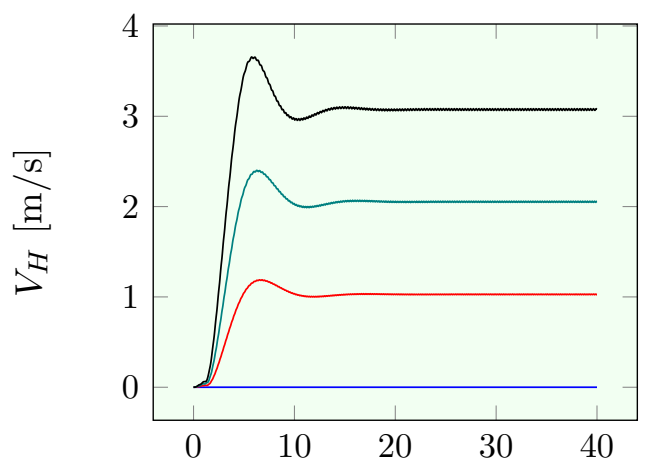

(d) $t[\mathrm{~s}]$

$$
-V_{H}=0 \mathrm{~m} / \mathrm{s}-V_{H}=1 \mathrm{~m} / \mathrm{s}-V_{H}=2 \mathrm{~m} / \mathrm{s}-V_{H}=3 \mathrm{~m} / \mathrm{s}
$$

Figura 48: Soluciones del sistema canónico con condiciones de control del sistema equilibrado 
La velocidad angular (figura 48c) presenta un comportamiento transitorio que varía su magnitud en función de la velocidad de desplazamiento pero que alcanza su equilibrio en aproximadamente el mismo intervalo de tiempo para todos los casos analizados. Por otra parte, también se observa un comportamiento dinámico de alta frecuencia, asociado a las fluctuaciones propias del momento en la dirección $z_{A}$.

Por último, en la figura 48d se comprueba que la velocidad de desplazamiento que adquiere el pararrotor es la buscada.

Para completar el análisis se calculan soluciones dinámicas para distintas configuraciones de equilibrio, a fin de evaluar el impacto de cada variable de estado en la solución que alcanza el sistema. Las figuras siguientes (figura 49) muestran valores convergidos del desempeño de $\mu_{z_{A}}, w_{T}$ y $\alpha_{b}$ en función de la velocidad de desplazamiento $V_{H}$ para distintos valores de ángulo de paso colectivo.

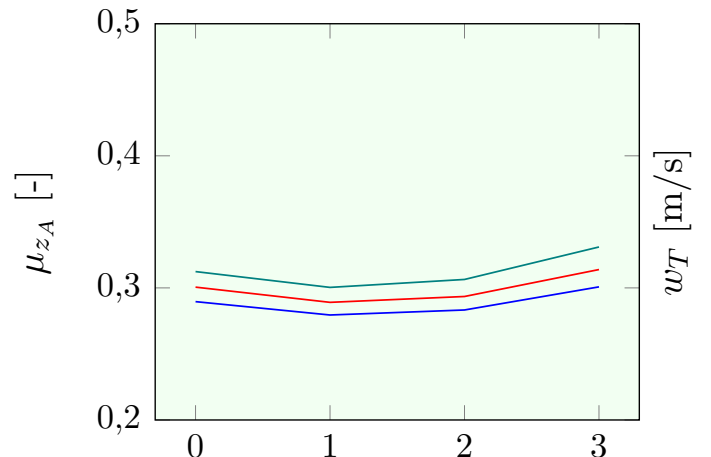

(a) $V_{H}[\mathrm{~m} / \mathrm{s}]$

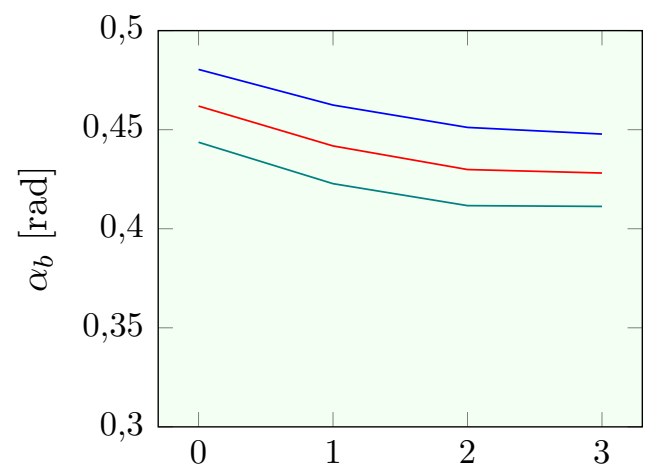

(c) $V_{H}[\mathrm{~m} / \mathrm{s}]$

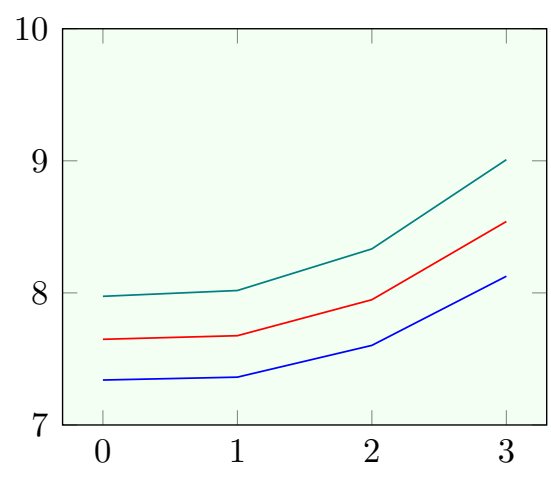

(b) $V_{H}[\mathrm{~m} / \mathrm{s}]$

$$
-\theta_{0}=2\left[^{\circ}\right]-\theta_{0}=4\left[^{\circ}\right]-\theta_{0}=6\left[^{\circ}\right]
$$

Figura 49: Valores caracterísiticos de distintos parámetros en el estado de equilibrio

Se observa que para un ángulo de paso colectivo determinado (figura 49a), $\mu_{z_{A}}$ varía levemente en función de la velocidad de traslación. Es decir que al 
aumentar la velocidad de desplazamiento $V_{H}$, la velocidad angular se modifica. El hecho de que disminuya el ángulo de paso colectivo al incrementarse la velocidad de traslación manteniendo constante la velocidad de descenso (figura 49b) confirma este comportamiento.

Este fenómeno puede interpretarse como que el sistema compensa el aumento del ángulo de paso (o la disminución del ángulo de ataque) de las palas mediante variaciones en $\omega_{z}$, de modo de mantener constante la fuerza de tracción generada para equilibrar al peso y mantener la operación del pararrotor en régimen de autorrotación. Esta compensación mantiene el equilibrio de fuerzas, manteniendo la velocidad de descenso $\left(w_{T}\right)$ deseada.

Desde otro punto de vista, se puede analizar el comportamiento del pararrotor en un régimen de descenso vertical ( $u_{T}$ y $v_{T}$ nulas) para distintas velocidades de descenso $w_{T}$. En la figura 50 se observa como disminuye el ángulo de ataque de la pala a medida que se incrementa la velocidad de descenso.

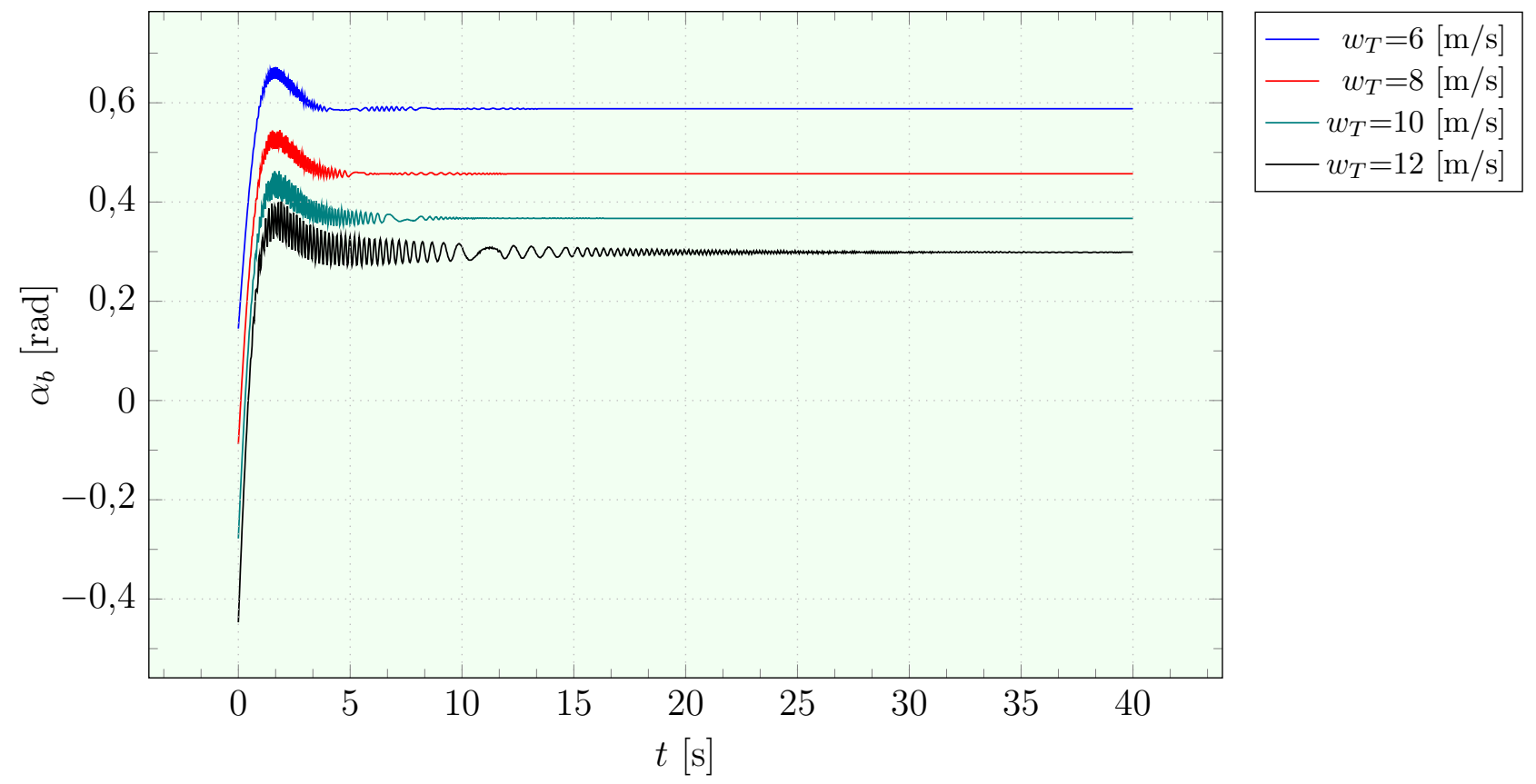

Figura 50: Ángulo de ataque de la pala $b$ en el sistema equilibrado

Según los trabajos de referencia [5] y [34], los coeficientes aerodinámicos pueden suponerse lineales para valores del ángulo de ataque $\alpha_{b}$ hasta $40^{\circ}(0,7 \mathrm{rad})$. Este valor se alcanza para velocidades de descenso $w_{T}=6 \mathrm{~m} / \mathrm{s}$, a partir de las cuales el ángulo de ataque disminuye y el modelo lineal implementado en la resolución tiene validez.

Atendiendo a esta observación, se puede explicar la disminución en $\alpha_{b}$ 
con el aumento de $w_{T}$ considerando que, para mantener el balance de fuerzas sobre el pararrotor aumentando la velocidad de descenso, es necesario reducir el ángulo de ataque para que la fuerza aerodinámica resultante se mantenga constante y persista el equilibrio que plantea la condición de autorrotación.

\subsubsection{Solución de la dinámica del pararrotor para distintas trayectorias de desplazamiento}

Por último, se presenta el caso de que se requiera la descripción de trayectorias laterales y longitudinales. Se calculan en primer término los vectores de estado extendido $\left(\mathbf{y}_{e}\right)$ y de control $\left(\mathbf{u}_{C_{e}}\right)$ en equilibrio, para luego ser utilizados en la solución de la dinámica completa.

El cuadro siguiente muestra las condiciones de vuelo que se reproducen:

\begin{tabular}{cc}
\hline \hline Parámetro & Valor \\
\hline$\beta_{T}$ & {$[45-90-135-180]\left[^{\circ}\right]$} \\
$w_{T}$ & $8 \mathrm{~m} / \mathrm{s}$ \\
$V_{H}$ & $3 \mathrm{~m} / \mathrm{s}$ \\
\hline \hline
\end{tabular}

Cuadro 16: Elementos prefijados del vector de estado

Para estas condiciones se tienen la siguientes soluciones de equilibrio:

- $\beta_{T}=45^{\circ}$

$$
\mathbf{y}_{e}=\left[\begin{array}{c}
u \\
v \\
w \\
\omega_{z} \\
\Theta \\
\Phi \\
u_{T} \\
v_{T} \\
w_{T} \\
\beta_{T} \\
V_{H}
\end{array}\right]=\left[\begin{array}{c}
2,9 \\
3,1 \\
7,4 \\
91,7 \\
-0,09 \\
0,13 \\
2,1 \\
2,1 \\
8 \\
0 \\
3
\end{array}\right], \mathbf{u}_{C_{e}}=\left[\begin{array}{c}
\theta_{0} \\
\theta_{1 C} \\
\theta_{1 S}
\end{array}\right]=\left[\begin{array}{c}
0,02 \\
0,1 \\
-0,08
\end{array}\right]
$$

- $\beta_{T}=90^{\circ}$ 


$$
\mathbf{y}_{e}=\left[\begin{array}{c}
u \\
v \\
w \\
\omega_{z} \\
\Theta \\
\Phi \\
u_{T} \\
v_{T} \\
w_{T} \\
\beta_{T} \\
V_{H}
\end{array}\right]=\left[\begin{array}{c}
-0,1 \\
4,2 \\
7,4 \\
91,7 \\
0,02 \\
0,16 \\
0 \\
3 \\
8 \\
0,5 \pi \\
3
\end{array}\right], \mathbf{u}_{C_{e}}=\left[\begin{array}{c}
\theta_{0} \\
\theta_{1 C} \\
\theta_{1 S}
\end{array}\right]=\left[\begin{array}{c}
0,02 \\
0,13 \\
0,008
\end{array}\right]
$$

- $\beta_{T}=135^{\circ}$

$$
\mathbf{y}_{e}=\left[\begin{array}{c}
u \\
v \\
w \\
\omega_{z} \\
\Theta \\
\Phi \\
u_{T} \\
v_{T} \\
w_{T} \\
\beta_{T} \\
V_{H}
\end{array}\right]=\left[\begin{array}{c}
-3,15 \\
2,8 \\
7,4 \\
91,7 \\
0,13 \\
0,09 \\
-2,1 \\
2,1 \\
8 \\
0,75 \pi \\
3
\end{array}\right], \mathbf{u}_{C_{e}}=\left[\begin{array}{c}
\theta_{0} \\
\theta_{1 C} \\
\theta_{1 S}
\end{array}\right]=\left[\begin{array}{c}
0,02 \\
0,08 \\
0,10
\end{array}\right]
$$

- $\beta_{T}=180^{\circ}$

$$
\mathbf{y}_{e}=\left[\begin{array}{c}
u \\
v \\
w \\
\omega_{z} \\
\Theta \\
\Phi \\
u_{T} \\
v_{T} \\
w_{T} \\
\beta_{T} \\
V_{H}
\end{array}\right]=\left[\begin{array}{c}
-4,25 \\
-0,1 \\
7,4 \\
91,7 \\
0,16 \\
-0,02 \\
-3 \\
0 \\
8 \\
\pi \\
3
\end{array}\right], \mathbf{u}_{C_{e}}=\left[\begin{array}{c}
\theta_{0} \\
\theta_{1 C} \\
\theta_{1 S}
\end{array}\right]=\left[\begin{array}{c}
0,02 \\
-0,008 \\
0,13
\end{array}\right]
$$

Debe aclararse que las velocidades se expresan en $[\mathrm{m} / \mathrm{s}]$, los ángulos en 
$[\mathrm{rad}]$ y las velocidades angulares en $[\mathrm{rad} / \mathrm{s}]$.

En base a estas soluciones de equilibrio se obtienen las siguientes figuras, que indican la evolución de las variables cinemáticas del pararrotor para cada una de las configuraciones propuestas:

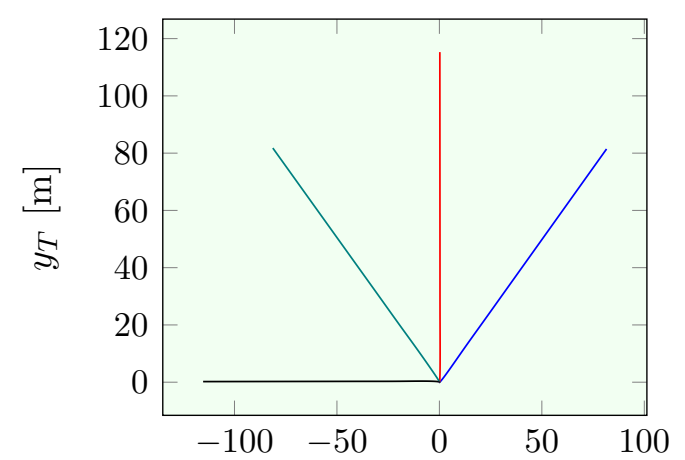

(a) $x_{T}[\mathrm{~m}]$

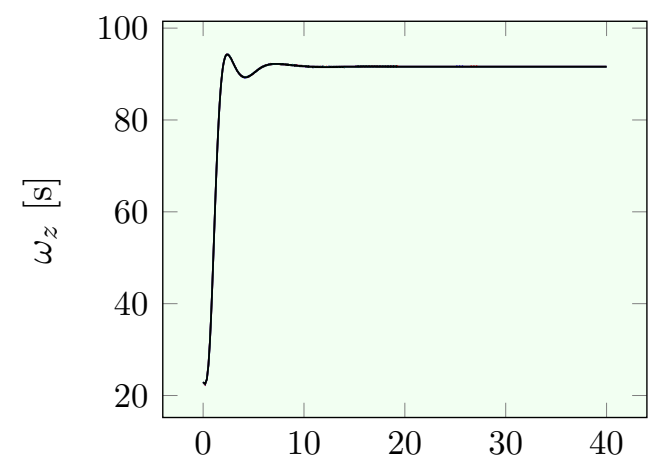

(c) $t[\mathrm{~s}]$

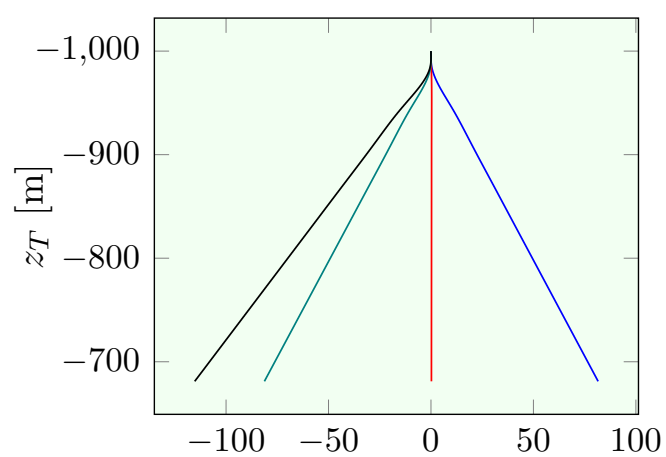

(b) $x_{T}[\mathrm{~m}]$

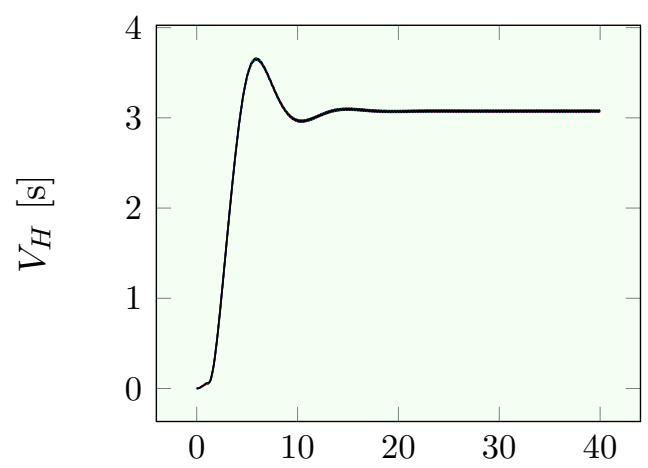

(d) $t[\mathrm{~s}]$

$-\beta_{T}=45^{\circ}-\beta_{T}=90^{\circ}-\beta_{T}=135^{\circ}-\beta_{T}=180^{\circ}$

Figura 51: Variables cinemáticas del pararrotor para distintas trayectorias

La observación de las figuras 51 permite comprobar que el modelo simplificado para la obtención del vector de control equilibrado obtiene valores del vector de control que generan la realización de la trayectoria deseada.

En la figura que representa el movimiento en el plano horizontal (figura 51a), resultan bien definidas las trayectorias para los ángulos deseados. El descenso del pararrotor (figura 51b) sigue distintos perfiles en virtud de la trayectoria requerida y, en función de esto, alcanza desplazamientos laterales mayores.

La velocidad angular (figura 51c) es representativa del movimiento cualitativo del pararrotor. Las curvas correspondientes a los desplazamientos quedan completamente superpuestas. Si se analiza el caso para el movimiento puramente 
horizontal o vertical (según la figura), esto puede explicarse debido a que el vector de control necesario para realizar una de las trayectorias intercambia con respecto al necesario para realizar la otra trayectoria sus primera y segunda componentes entre sí, ya que cada una de las componentes (lateral o longitudinal) domina el movimiento del pararrotor según el plano correspondiente. El mismo análisis explica el comportamiento de la velocidad angular para las trayectorias que corresponden a $\beta_{T}=45^{\circ}$ y $\beta_{T}=135^{\circ}$.

Naturalmente, la velocidad de desplazamiento horizontal (figura 51d) es la misma para todos los casos, por lo que no se observan diferencias según la trayectoria.

\subsection{Conclusiones del capítulo}

A partir del desarrollo del presente capítulo puede afirmarse que es posible hallar configuraciones operativas próximas al equilibrio para un pararrotor.

Se comprueba también que se obtienen soluciones de equilibrio a partir de suponer acciones promediadas en la vuelta, y que estas soluciones determinan el comportamiento deseado al ser utilizadas para resolver la ecuación de la dinámica del pararrotor. El análisis de estas soluciones brinda elementos de diagnóstico que explican el comportamiento general del pararrotor.

Partiendo del sistema de ecuaciones de la dinámica del pararrotor, desarrollada en su forma canónica, se obtienen soluciones del vector de estado en función del vector de control que determinan la trayectoria buscada para el centro de masas del pararrotor de manera unívoca. Como se observa en los casos de análisis presentados, existen configuraciones del vector de control que determinan la trayectoria deseada: es posible describir trayectorias rectas en el plano $x_{T}-y_{T}$ a partir de fijar el vector de control $\mathbf{u}_{C}$, incluso con componentes de viento atmosférico distintas de cero. Además, las componentes del vector de control presentan un comportamiento no lineal respecto a la velocidad de desplazamiento, que permite establecer, según el caso, acciones de control de distinta naturaleza.

Con estas conclusiones, aparece un abánico de posibilidades de desarrollo del estudio de las trayectorias que no se brinda en el presente trabajo pero establece basamento para abordar el estudio de la estabilidad del sistema, la optimización de la trayectorias y el problema del control en tiempo real de los pararrotores. El estudio de la estabilidad, en el caso de la utilización de la variación cíclica del paso de las palas, puede orientarse inicialmente hacia la linealización del sistema alrededor de un punto de equilibrio, en el que la tasa de cambio temporal del vector de estado sea proporcional al vector de estado a través de una matriz de estabilidad periódica. 


\section{Conclusiones}

\subsection{Introducción}

El objetivo primordial del presente trabajo fue el estudio del desplazamiento lateral de un pararrotor mediante variaciones cíclicas del paso de las palas. Se abordó este problema de modo integral, desglosando su análisis entre los aspectos experimentales y teóricos. El pequeño alargamiento de las palas de los pararrotores convierten a este estudio en un caso particular dentro del régimen de autorrotación, ya que se desarrollan efectos tridimensionales que en otros casos de estudio no son considerados.

El presente capítulo recoge las principales conclusiones desarrolladas en cada capítulo, describiendo su interrelación, los aportes novedosos y planteando líneas futuras para la profundización en el estudio de los pararrotores.

La presente tesis brinda continuidad en las investigaciones y desarrollos sobre pararrotores, y, particularmente, da cuenta de los efectos que provoca la variación cíclica del ángulo de paso de las palas en el régimen de operación de los mismos.

\subsubsection{Modelo teórico}

El modelo teórico, desarrollado a partir de las ecuaciones de NewtonEuler para un pararrotor, relaciona las fuerzas y momentos que actúan sobre el modelo bajo estudio. Tal como se menciona en el correspondiente capítulo, se desglosó el modelado de los efectos inerciales, aerodinámicos y gravitatorios, obteniéndose representaciones matemáticas que permitieron analizar el efecto de la modificación de las variables de estado y de control a partir de modelos simplificados obtenidos mediante desarrollos en serie de Taylor. El modelo dinámico completo, representado sintéticamente en la ecuación (41), fue resuelto a partir de la obtención de la formulación canónica del sistema de ecuaciones. Se obtuvo una representación canónica de las ecuaciones de Newton-Euler, junto a las condiciones de ligadura cinemática y la 
ecuación de trayectoria del centro de masas del pararrotor, que fue implementada en un código de resolución numérica. En particular, se obtuvo la solución del sistema de ecuaciones reducido al modelo en túnel de viento.

El modelo simplificado, obtenido a partir de los desarrollos en series de Taylor para los efectos inerciales y aerodinámicos, también fue resuelto y sus resultados fueron comparados con los obtenidos por el modelo completo. Las principales conclusiones que se pueden comentar son:

- la velocidad angular $(\Omega)$ del modelo simplificado excede en un $20 \%$ a la que se obtiene mediante el modelo completo, posiblemente debido a efectos de segundo orden no contemplados en el modelo aerodinámico.

- el tiempo de establecimiento de la solución estacionaria es mayor para el modelo simplificado.

- los momentos inerciales ocurren cuando se aplica un ángulo de paso cíclico. Estos momentos son, para $\theta_{1 S}$ y $\theta_{1 C}$, lineales según los ejes $x_{A}$ e $y_{A}$ y cuadráticos según $z_{A}$.

- los momentos aerodinámicos según $x_{A}$ e $y_{A}$ son dominados por la variación cíclica del paso de las palas.

- el momento en el eje $z_{A}$ depende del ángulo de paso colectivo, y del coeficiente $\mu_{z_{A}}$.

La validación del modelo teórico sirvió de base para la extracción de conclusiones adicionales a partir de la interpretación del mismo:

- se demostró la pequeña relación entre los órdenes de magnitud de los momentos de origen inercial con los aerodinámicos.

- se obtuvieron valores del momento en el punto de acoplamiento $E_{b}$, que pueden ser utilizados en el diseño de los acoples y los servomecanismos de operación.

Basado en las anteriores conclusiones parciales, puede decirse que es posible controlar fuerzas y momentos sobre el pararrotor mediante la variación cíclica del ángulo de paso de las palas. Esta conclusión responde afirmativamente a la pregunta de si es posible controlar la trayectoria del pararrotor utilizando una estrategia de control sobre el ángulo de paso cíclico de las palas.

\subsubsection{Modelo experimental}

Se diseñó y construyó un modelo experimental sobre el que se estudió el efecto de las variaciones cíclicas del paso de las palas en la fuerza lateral generada 
sobre un banco de pruebas ad hoc.

Los parámetros seleccionados para el análisis del comportamiento dinámico del pararrotor en el túnel de viento fueron la velocidad angular $\Omega$ y la fuerza lateral $F$.

Se observa que el incremento en el ángulo de paso cíclico provoca un aumento en la fuerza lateral $F$ que genera el pararrotor. Esto se debe a que los ángulos de ataque a los que opera el pararrotor aumentan con el ángulo de paso cíclico, traduciéndose en valores medios del momento sobre el cuerpo del pararrotor mayores.

Puede decirse que la disminución de la velocidad angular según el aumento en el ángulo de paso cíclico se debe al incremento en la resistencia ante mayores ángulos de ataque. Este dato complementa investigaciones previas, donde se analizó el comportamiento del parámetro $\mu_{z_{A}}$ para ángulos de paso cíclico nulo. En estos ensayos, $\mu_{z_{A}}$ disminuye conforme aumenta $\theta_{0}$.

\subsubsection{Simulación de trayectorias}

El estudio de las trayectorias de descenso permite establecer que existen configuraciones del vector de control que se traducen en estados de operación cercanos al equilibrio. En particular, es posible definir un vector de control para describir una trayectoria deseada.

El modelo de acciones promediadas en la vuelta resulta aceptable. Las leyes de control obtenidas a partir de las soluciones de equilibrio son utilizadas en el modelo dinámico completo y brindan soluciones próximas al equilibrio para las variables de estado.

Se observa que los parámetros de $\theta_{1 S}$ y $\theta_{1 C}$ tienen un comportamiento no lineal en función de la velocidad de desplazamiento y del ángulo de dirección horizontal. Existen distintas regiones donde la pendientes de las curvas cambian de signo, por lo tanto la naturaleza de la acción de control depende del régimen de operación en el que se encuentra el pararrotor. El parámetro $\theta_{0}$ tiene un comportamiento no lineal para valores bajos de $V_{H}$, y pude aproximarse lineal para $V_{H}>=2$ $\mathrm{m} / \mathrm{s}$.

\subsubsection{Futuras líneas de investigación}

En primer lugar, resulta de interés la profundización del desarrollo teórico, puntualizando en aspectos que fueron simplificados en el actual trabajo: 
- el estudio y la implementación de modelos aerodinámicos de mayor complejidad, suponiendo coeficientes de sustentación y resistencia que representen con mayor fidelidad el comportamiento tridimensional del flujo.

- el estudio y la implementación de modelos para la velocidad inducida, mejorando el modelo de disco actuador y la teoría de cantidad de movimiento utilizada.

- el estudio y la implementación de modelos aerodinámicos para los efectos que introduce el cuerpo cilíndrico del pararrotor.

En cuanto al desarrollo experimental, resulta interesante desarrollar técnicas de medición que apunten a obtener mayor información del comportamiento de las variables medidas (velocidad angular y fuerza lateral) a lo largo de una vuelta. Por ejemplo:

- implementación de un sistema de medición de la velocidad angular a lo largo de la vuelta, para poder comparar dichos perfiles con los modelos teóricos y perfeccionar los estudios realizados.

- implementación de un sistema de medición de fuerzas de 6 ejes, para la adquisición de las fuerzas y momentos en los 6 grados de libertad que tiene el pararrotor como sólido rígido.

También se pueden trazar líneas tendientes a la implementación de este tipo de estrategias de control en modelos de vuelo. El desarrollo de un mecanismo de variación cíclica del ángulo de las palas en un modelo de vuelo plantea ya de por sí desafíos experimentales, ya que en vuelo, el pararrotor (como cualquier otro aparato volador) no dispone de puntos de soporte en los que fijar un plato oscilante.

La simulación de trayectorias plantea perspectivas amplias de desarrollo. El enfoque trabajado en esta tesis resulta en un aporte novedoso en su utilización sobre pararrotores, desde el que pueden desprenderse distintas alternativas de desarrollo:

- el diseño y la implementación de un sistema de control de trayectoria en tiempo real, sensible a posibles perturbaciones externas.

- la continuidad en el estudio de la estabilidad, incorporando el análisis de matrices periódicas en función de los parámetros de control cíclicos.

- el estudio de técnicas de optimización de trayectorias, para la determinación de controles cíclicos que den lugar a trayectorias de descenso de determinadas características. 


\subsection{Aportes originales de la tesis}

Los aportes originales de este trabajo pueden condensarse en:

- el desarrollo de un modelo dinámico analítico del pararrotor, contemplando el efecto de las variaciones cíclicas en el ángulo de paso de las palas,

- la implementación en un código de resolución numérica del modelo analítico completo,

- el desarrollo de modelos simplificados para las fuerzas y momentos que aparecen en este tipo de dispositivos,

- el estudio experimental del efecto de las variaciones cíclicas en el paso de las palas sobre parámetros relevantes,

- la identificación de acciones de control para la obtención de determinadas trayectorias. 


\section{Anexos}

\subsection{Caracterización aerodinámica de las palas}

En este apartado se aborda el problema vinculado a la determinación de los coeficientes aerodinámicos necesarios para la evaluación del modelo teórico. Es necesario destacar que la utilización de las palas de bajo alargamiento introduce efectos no lineales que deben ser evaluados. Para el comportamiento de la posición del centro de presiones según la cuerda del ala desde el borde de ataque se adopta la distribución propuesta en [35], que modela la distribución de la diferencia de presiones para cualquier tipo de ala mediante la siguiente expresión:

$$
\Delta C_{p}(x)=\frac{-\sin (\pi n)}{\pi n} C_{L}\left(\frac{1-x}{x}\right)^{n}
$$

El parámetro $n$, en el caso de $A R<1$, varía según la ley:

$$
n=1-\sqrt{\frac{A R}{8}}
$$

y la posición del centro de presiones asociada a este modelo resulta:

$$
x_{c p}=\frac{1-n}{2} \cdot 100 \%
$$

Cabe observar que la ecuación (116) representa una familia de funciones de distribución de carga a lo largo de la cuerda que incluye los casos extremos, cuyos resultados son conocidos: la posición del centro de presiones se encuentra al $25 \%$ de la cuerda cuando $\mathrm{AR} \rightarrow \infty$, y es 0 cuando $\mathrm{AR} \rightarrow 0$.

El gráfico siguiente muestra la distribución de la diferencia de presiones para distintos ángulos de ataque: 


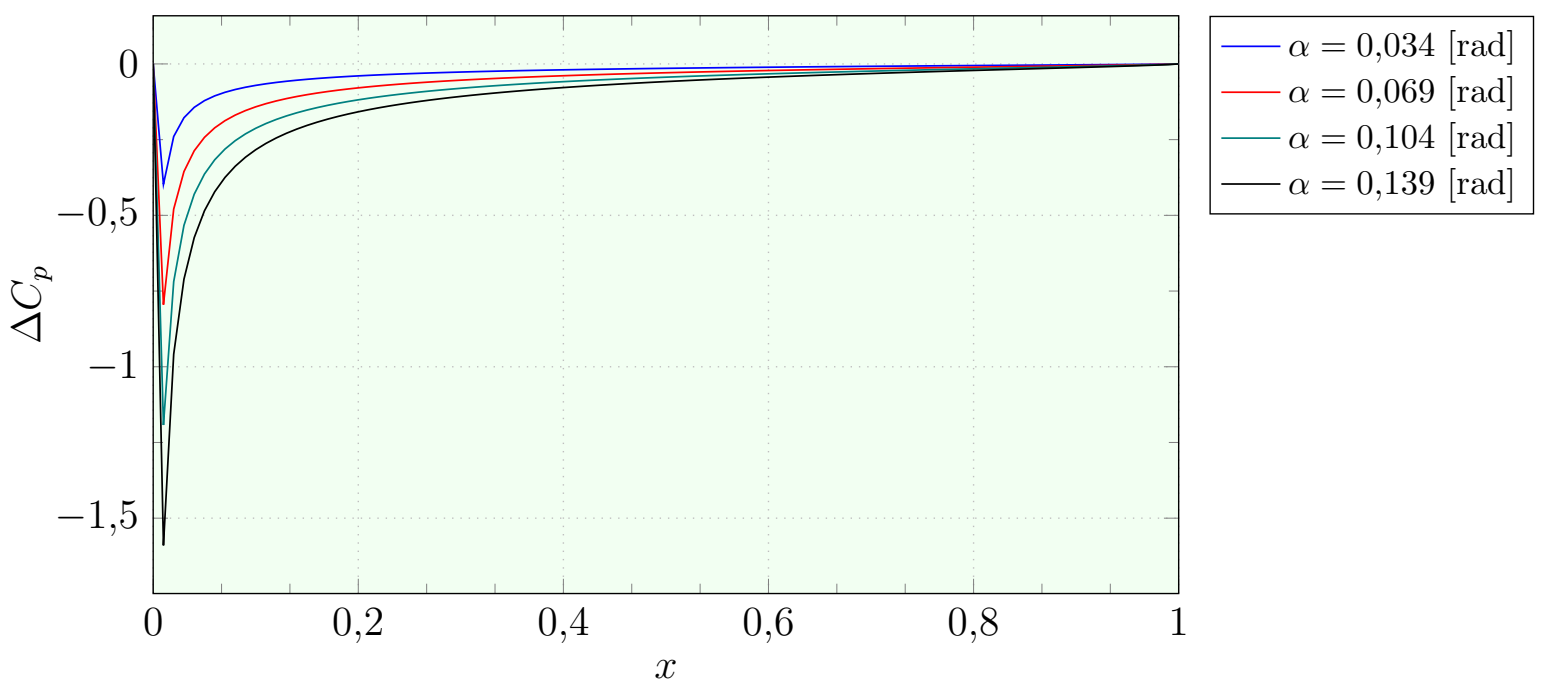

Figura 52: Distribución del coeficiente de presiones sobre una placa plana.

Existen distintos modelos para la representación de los coeficientes aerodinámicos $C_{D}$ y $C_{L}$ [6]. Si bien las palas de pequeño alargamiento presentan un comportamiento no lineal para sus coeficientes aerodinámicos, se puede calcular la pendiente inicial de la curva $C_{l \alpha}$ vs $\alpha$ a partir del ajuste realizado en la curva de sustentación. Esta pendiente es comparada con otros modelos lineales. Para la pendiente del coeficiente de sustentación, el primer modelo se deriva de la teoría de línea sustentadora de Prandtl:

$$
C_{L \alpha}=\frac{C_{l \alpha}}{1+\frac{C_{l \alpha}}{\pi A R}(1+\tau)}[1 / \mathrm{rad}]
$$

Donde $\tau$ es el coeficiente de eficiencia de Glauert, que varía entre 0,05 y 0,25 . El valor de $C_{l \alpha}$ es $C_{l \alpha}=5,374[1 / \mathrm{rad}]$ que corresponde al valor promedio de los experimentos realizados en [6] para placas planas infinitas. Otro modelo es el de Lowry y Polhamus, que es más preciso para $\mathrm{AR} \leq 2$ :

$$
C_{L \alpha}=\frac{2 \pi A R}{2+\sqrt{\frac{A R^{2}}{\eta^{2}}\left(1+\tan ^{2} \Lambda\right)+4}}[1 / \mathrm{rad}]
$$

Por último, se presenta el modelo de Hoerner y Borst, que para alas rectangulares delgadas de bajo alargamiento es:

$$
C_{L \alpha}=\left[\frac{36,5}{A R}+2 A R\right]^{-1}
$$

La utilización de estos modelos implica asumir un comportamiento lineal para la sustentación. Polhamus [6] propone el siguiente modelo no lineal para el 
coeficiente de sustentación: ${ }^{2}$

$$
C_{L}=K_{p} \sin \alpha \cos ^{2} \alpha+K_{v} \cos \alpha \sin ^{2} \alpha
$$

donde $K_{p}$ es un factor que depende del alargamiento, el ángulo de flecha y la forma del borde de ataque; y $K_{v}$ un factor aproximadamente constante cercano a $\pi$. En el caso de la estimación del coeficiente de resistencia, la siguiente ecuación no lineal fue propuesta por Lamar:

$$
C_{D}=C_{D 0}+K_{p} \sin ^{2} \alpha \cos \alpha+K_{v} \sin ^{3} \alpha
$$

Sin embargo, el ajuste a los datos experimentales de este modelo no resultó adecuado. Un mejor ajuste se encuentra utilizando la ecuación clásica de resistencia inducida:

$$
C_{D}=C_{D 0}+K\left[C_{L}\right]^{2}
$$

siendo $K$ el factor de resistencia inducida, parámetro que depende del alargamiento, la forma de la pala y de la forma del borde de ataque.

Ortiz et. al [34] presenta una distribución para el coeficiente de resistencia según el alargamiento en la que se alcanzan ángulos de ataque de $45^{\circ}$. Para una pala como la del pararrotor bajo estudio $(\mathrm{AR}=0,63)$, se tiene la siguiente distribución:

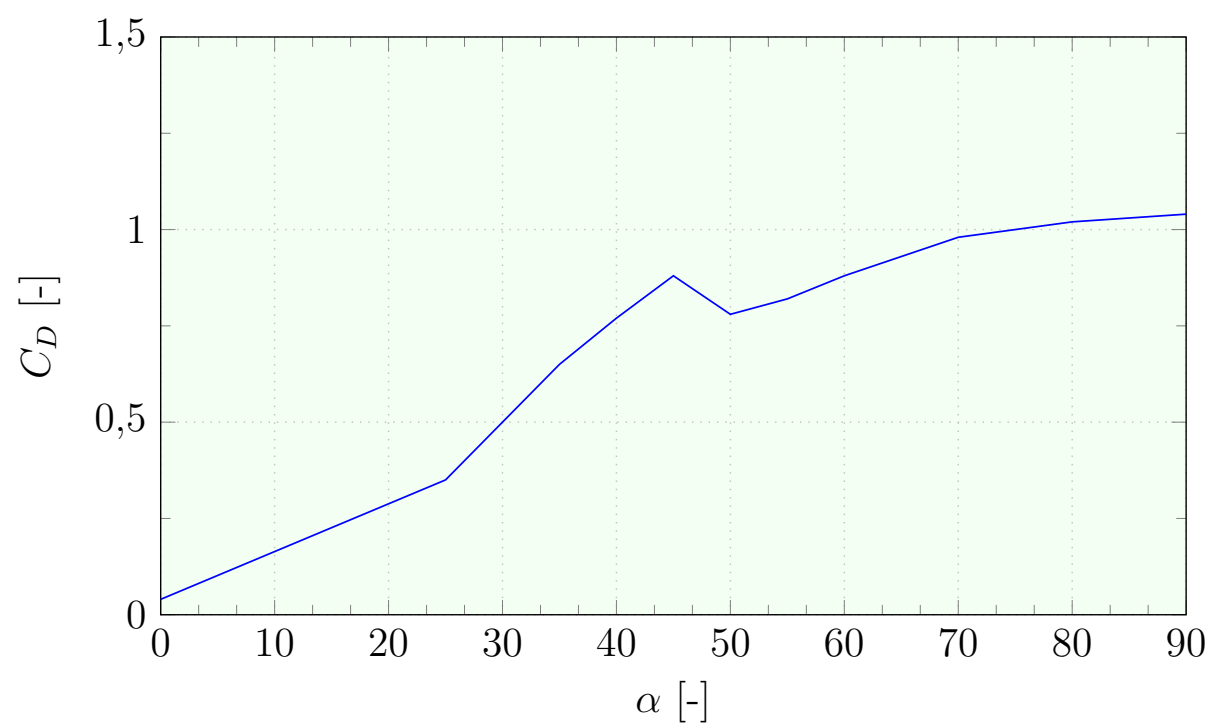

Figura 53: Modelo de coeficiente de resistencia [34]

En principio se analiza la implementación de un modelo aerodinámico simple, en el que los coeficientes de sustentación y resistencia sean los siguientes:

$$
C_{L}(\alpha, \beta)=C_{L_{\alpha}} \alpha
$$

\footnotetext{
${ }^{2}$ si bien esta expresión es desarrollada para alas tipo delta, la elección adecuada de los coeficientes permite utilizarla para alas rectangulares
} 


$$
C_{D}(\alpha, \beta)=C_{D}
$$

La variedad de métodos que se han desarrollado para la caracterización aerodinámica de las palas de bajo alargamiento muestra que es un aspecto sensible a tener en cuenta para el modelo aerodinámico del pararrotor. Sobre todo teniendo en consideración que el régimen de autorrotación en el que operan estos dispositivos establece una configuración aerodinámica que no es estudiada profundamente en la bibliografía de referencia, por lo que estos parámetros deben ser ajustados atentos a los resultados experimentales.

\subsection{Obtención de expresiones matemáticas para el desarrollo en serie de Taylor del modelo en túnel de viento}

El presente apartado tiene como objetivo describir la obtención de las expresiones correspondientes para las fuerzas y momentos, tanto inerciales como aerodinámicas o gravitatorias. En el capítulo 3 se obtienen dichas expresiones de forma simplificada, a partir de las cuales se realizan observaciones que permiten categorizar los efectos que introducen los parámetros de control. A continuación se describe la obtención de dichas ecuaciones para el caso de los términos inerciales, seguida de la descripción de la obtención de los términos aerodinámicos y los gravitatorios.

\subsubsection{Obtención de términos inerciales}

La obtención de expresiones matemáticas para los términos de origen inercial se divide según los cuerpos que conforman un pararrotor. Primero se obtiene la formulación correspondiente para el cuerpo del pararrotor, y a continuación, las expresiones para las palas del pararrotor.

\subsubsection{Fuerzas y momentos inerciales del cilindro del pararrotor}

Las variables de estado, definidas en la sección 3.4, aparecen en las expresiones de las fuerzas y momentos inerciales a través de las aceleraciones y velocidades que las definen. En este apartado, de desarrolla de modo general la obtención de las expresiones matemáticas, para ser utilizadas en el modelo dinámico completo y, también, para la realización de desarrollos en series de Taylor a partir de la asignación de ordenes de magnitud para cada una de las variables.

Las fuerzas inerciales que genera el cilindro del pararrotor, denominado $H$, son originadas debido a las aceleraciones lineales del mismo. Para cuantificarlas, pueden definirse como: 


$$
\mathbf{F}_{H}^{i}=-M_{H} \cdot \mathbf{a}^{G}
$$

La aceleración del centro de masas del cilindro del pararrotor se determina a partir de calcular la derivada absoluta respecto al tiempo de la velocidad del centro de masas del pararrotor expresada en el sistema $\mathrm{A} 1 \mathrm{~b}\left(\left.\mathbf{V}^{G}\right|_{A 1 b}\right)$, y expresándola en el sistema A:

$$
\left.\mathbf{a}^{G}\right|_{A 1 b}=\frac{\left.d \mathbf{V}^{G}\right|_{A 1 b}}{d t}+\left.\left.\boldsymbol{\omega}\right|_{A 1 b} \wedge \mathbf{V}^{G}\right|_{A 1 b}
$$

La expresión de esta aceleración en el sistema A se realiza mediante la transformación siguiente:

$$
\left.\mathbf{a}^{G}\right|_{A}=\left.\mathbf{T}_{A A 1 b} \mathbf{a}^{G}\right|_{A 1 b}
$$

Reemplazando en la expresión (126) se obtiene la representación en función de las variables de estado para las fuerzas, que permite obtener los coeficientes $\mathbf{M}_{H}^{F}$ necesarios para la formulación canónica de la ecuación de Newton realizada en el apartado 3.9.1 .

Por medio de la ecuación de Euler pueden cuantificarse los momentos inerciales que genera el cilindro del pararrotor. En el caso general, se tiene la siguiente formulación:

$$
M_{H}^{i}=-\frac{d \mathbf{h}_{H}}{d t}
$$

Asimismo, el momento cinético del cilindro del pararrotor en ejes A1b puede expresarse como:

$$
\mathbf{h}_{H}=\left.I_{H}\right|_{A 1 b} \cdot \boldsymbol{\omega}_{A 1 b}
$$

La derivada absoluta de esta ecuación, expresada en ejes A1b, es la que permite obtener expresiones para los coeficientes $\mathbf{M}_{H}^{M}$ en función de las variables de estado, que son utilizadas en el apartado 3.9.2.

\subsubsection{Fuerzas y momentos inerciales de las palas}

Para las palas, el procedimiento es el mismo que para el caso del cilindro del pararrotor. En primer lugar, se busca determinar analíticamente la fuerza inercial que introducen. Para esto se busca la expresión correspondiente a la aceleración del 
centro de masas de la pala $b\left(\mathbf{a}^{G B b}\right)$, a partir de la derivada absoluta de la velocidad del centro de masas:

$$
\left.\mathbf{a}^{G B b}\right|_{B_{b}}=\frac{\left.d \mathbf{V}^{G B b}\right|_{B_{b}}}{d t}+\left.\left.\boldsymbol{\omega}_{b}\right|_{B_{b}} \wedge \mathbf{V}^{G B b}\right|_{B_{b}}
$$

Su expresión, en el sistema de referencia A, se obtiene premultiplicando por las matrices de transformación correspondientes:

$$
\left.\mathbf{a}^{G B b}\right|_{A}=\left.\left[\mathbf{T}_{A A 1 b}\right]\left[\mathbf{T}_{A 1 b B_{b}}\right] \mathbf{a}^{G B b}\right|_{B b}
$$

La fuerza inercial se define como:

$$
\mathbf{F}_{b}^{i}=-M_{p} \cdot \mathbf{a}^{G B b}
$$

La fuerza inercial resultante de las dos palas se calcula sumando cada una de las contribuciones de las mismas:

$$
\mathbf{F}_{B}^{i}=\mathbf{F}_{1}^{i}+\mathbf{F}_{2}^{i}
$$

A partir del desarrollo de esta ecuación se pueden identificar los términos de la submatriz $\mathbf{M}_{B}^{F}$, utilizados en el apartado 3.9.1.

La expresión general de los momentos inerciales que introducen las palas se obtienen a partir de definir el momento cinético respecto al punto $E_{b}$ (punto de acoplamiento de la pala $b$ ):

$$
\left.\mathbf{h}_{b}\right|_{B_{b}}=\left.I_{B} \cdot \boldsymbol{\omega}_{b}\right|_{B_{b}}
$$

El momento inercial se relaciona con la derivada temporal absoluta de $\left.\mathbf{h}_{b}\right|_{B b}$ como se muestra en la siguiente ecuación:

$$
\mathbf{M}_{B_{b}}^{i, E_{b}}=-\frac{\left.d \mathbf{h}_{b}\right|_{B b}}{d t}-\left.\boldsymbol{\omega}_{B b} \wedge \mathbf{h}_{b}\right|_{B b}
$$

Para obtener su expresión en ejes A es necesario premultiplicar por la matriz $\left[\mathbf{T}_{A A 1 b}\right]\left[\mathbf{T}_{A 1 b B_{b}}\right]$ :

$$
\mathbf{M}_{A}^{i, E_{b}}=\left[\mathbf{T}_{A A 1 b}\right]\left[\mathbf{T}_{A 1 b B_{b}}\right] \mathbf{M}_{B_{b}}^{i, E_{b}}
$$

Finalmente, para lograr su expresión respecto al centro de masas del pararrotor (punto $\mathrm{G}$ ), se añaden los momentos que hacen las fuerzas inerciales respecto a $\mathrm{G}$ :

$$
\mathbf{M}_{b, A}^{i, G}=\mathbf{M}_{A}^{i, E_{b}}+\left(\left.\left.\mathbf{G E}_{b}\right|_{A} \wedge \mathbf{F}_{b}^{i}\right|_{A}\right)
$$


Nuevamente, se llega a una expresión a partir de la cual se pueden extraer los coeficientes $\mathbf{M}_{B}^{M}$ en función de las variables de estado para la expresión canónica desarrollada en 3.9.2 .

Las expresiones desarrolladas para las fuerzas y momentos inerciales de las palas y el cilindro se encuentran presentadas en forma vectorial debido a la complejidad y extensión que presentan según sus componentes. A los efectos de obtener expresiones que permitan el análisis cualitativo de la influencia de los parámetros que la componen, se realiza el desarrollo en series de Taylor que se presenta en el apartado 3.11.1, particularmente para el caso del modelo en túnel de viento.

Suponer el pararrotor montado en el túnel de viento implica realizar una serie de simplificaciones en las condiciones operativas que se traducen, matemáticamente, en expresiones más sencillas. Se puede asumir que la velocidad angular $\boldsymbol{\omega}$ tiene solamente una componente, es decir $\boldsymbol{\omega}=\omega_{z} \mathbf{k}_{A}$. También se asume que la velocidad del centro de masas es nula $\left(\mathbf{V}^{G}=\mathbf{0}\right)$, que la velocidad del viento tiene solamente componente según el eje del túnel de viento $\left(\mathbf{U}_{W}=\mathbf{U} \mathbf{k}_{A}\right)$ y que la velocidad inducida es nula $\left(\mathbf{v}_{i}=\mathbf{0}\right)$. Por otro lado, la geometría del pararrotor se puede simplificar, suponiendo que las palas se encuentran contenidas en el plano que contiene al centro de masas (por lo tanto el centro de presiones de las mismas, punto $P_{b}$ ).

Estas simplificaciones permiten encontrar la siguiente expresión para las fuerzas inrciales, aplicadas en el centro de masas de la pala y expresadas en el sistema A:

$$
\begin{gathered}
\left.F_{b, x_{A}}^{i}=-M_{b}\left[-\cos \psi_{b} \frac{d \psi_{b}}{d t} \Omega\left(e+x_{G B}\right)-\sin \psi_{b} \frac{d \Omega}{d t}\left(e+x_{G B}\right)\right)\right] \\
\left.F_{b, y_{A}}^{i}=-M_{b}\left[-\sin \psi_{b} \frac{d \psi_{b}}{d t} \Omega\left(e+x_{G B}\right)+\cos \psi_{b} \frac{d \Omega}{d t}\left(e+x_{G B}\right)\right)\right] \\
F_{b, z_{A}}^{i}=0
\end{gathered}
$$

Del mismo modo se obtiene la expresión de los momentos inerciales en el sistema de referencia A:

$$
\begin{aligned}
M_{x_{A}}^{i, E_{b}}= & -I_{x_{B}}\left[\cos \psi_{b} \frac{d \psi_{b}}{d t} \sin \theta_{b} \cos \theta_{b} \Omega+\sin \psi_{b} \frac{d \theta_{b}}{d t} \cos ^{2} \theta_{b} \Omega(t)\right. \\
& +\sin \psi_{b} \frac{d \Omega}{d t} \sin \theta_{b} \cos \theta_{b}-\sin \psi_{b} \frac{d \theta_{b}}{d t} \sin ^{2} \theta_{b} \Omega \\
& +\frac{d^{2} \theta_{b}}{d t} \cos \psi_{b}-\sin \psi_{b} \frac{d \theta_{b}}{d t} \frac{d \psi_{b}}{d t}
\end{aligned}
$$




$$
\begin{aligned}
M_{y_{A}}^{i, E_{b}}= & -I_{x_{B}}\left[\sin \psi_{b} \frac{d \psi_{b}}{d t} \sin \theta_{b} \cos \theta_{b} \Omega-\cos \psi_{b} \frac{d \theta_{b}}{d t} \cos ^{2} \theta_{b} \Omega(t)\right. \\
& -\cos \psi_{b} \frac{d \Omega}{d t} \sin \theta_{b} \cos \theta_{b}-\cos \psi_{b} \frac{d \theta_{b}}{d t} \sin ^{2} \theta_{b} \Omega \\
+ & \frac{d^{2} \theta_{b}}{d t} \sin \psi_{b}+\cos \psi_{b} \frac{d \theta_{b}}{d t} \frac{d \psi_{b}}{d t} \\
M_{z_{A}}^{i, E_{b}}= & -\left(\cos ^{2} \theta_{b} I_{x_{B}}+I_{y_{B}}\right) \frac{d \Omega}{d t}+2 I_{x_{B}} \Omega \cos \theta_{1} \sin \theta_{b} \frac{d \theta_{b}}{d t} \\
& -M_{b}\left[x_{G B_{b}} e \cos \psi_{b}\left(-\sin \psi_{b} \frac{d \psi_{b}}{d t} \Omega+\cos \psi_{b} \frac{d \Omega}{d t}\right)\right. \\
& -\sin \psi_{b G B_{b}} e\left(-\cos \psi_{b} \frac{d \psi_{b}}{d t} \Omega-\sin \psi_{b} \frac{d \Omega}{d t}\right)
\end{aligned}
$$

Sobre las ecuaciones (139) y (140) se realizó el desarrollo en series de Taylor multivariable, definiendo los siguientes órdenes de magnitud para cada una de las variables seleccionadas:

\begin{tabular}{cc}
\hline \hline Parámetro & Orden de magnitud \\
\hline$\theta_{0}[\mathrm{rad}]$ & 1 о 2 \\
$\theta_{1 S}[\mathrm{rad}]$ & 1 о 2 \\
$\theta_{1 C}[\mathrm{rad}]$ & 1 о 2 \\
$\mu_{z_{A}}[-]$ & 1 о 2 \\
$e[\mathrm{~m}]$ & 1 \\
$x_{G B}$ e $y_{G B}[\mathrm{~m}]$ & 1 \\
\hline \hline
\end{tabular}

Cuadro 17: Órdenes de magnitud para los desarrollos en series de Taylor de fuerzas y momentos inerciales

Las ecuaciones (78), (79) y (80) se obtienen realizando el desarrollo en series de Taylor con los órdenes de magnitud del cuadro 17.

\subsubsection{Obtención de términos aerodinámicos}

Para el cuerpo cilíndrico del pararrotor se supone que las fuerzas y momentos aerodinámicos son nulos, tal como se justifica en el apartado 5.3.1.

Las fuerzas aerodinámicas (ecuaciones (58) y (59)), generadas en el régimen de autorrotación del pararrotor, se encuentran expresadas en el sistema de referencia viento, ya que, naturalmente, es más sencillo hallar las expresiones de las mismas en este sistema de referencia. La transformación al sistema de referencia de la pala se obtiene premultiplicando por las matrices correspondientes, tal como se 
observa en la ecuación (60). La expresión que se obtiene al desarrollar dicha ecuación es la siguiente:

$$
\begin{gathered}
F_{b, x_{B_{b}}}^{a}=0 \\
F_{b, y_{B_{b}}}^{a}=\frac{1}{2} \rho S\left[\left(\left(\cos \theta_{b} \Omega y_{P_{b}}\right)^{2}+\left(-\cos \theta_{b} \Omega\left(e+x_{P_{b}}\right)-\sin \theta_{b} U_{W}\right)^{2}\right.\right. \\
+\left(\sin \theta_{b} \Omega\left(e+x_{P_{b}}\right)-\frac{d \theta_{b}}{d t} y_{P_{b}}-\cos \theta_{b} U_{W}\right)^{2}\left(\cos \alpha_{b} C_{D}+\sin \alpha_{b} C_{L_{\alpha_{b}}}\right) \\
F_{b, z_{B_{b}}}^{a}=\frac{1}{2} \rho S\left[\left(\left(\cos \theta_{b} \Omega y_{P_{b}}\right)^{2}+\left(-\cos \theta_{b} \Omega\left(e+x_{P_{b}}\right)-\sin \theta_{b} U_{W}\right)^{2}\right.\right. \\
+\left(\sin \theta_{b} \Omega\left(e+x_{P_{b}}\right)-\frac{d \theta_{b}}{d t} y_{P_{b}}-\cos \theta_{b} U_{W}\right)^{2}\left(\sin \alpha_{b} C_{D}+\cos \alpha_{b} C_{L_{\alpha_{b}}}\right)
\end{gathered}
$$

El momento aerodinámico de las palas se calcula multiplicando el vector posición del centro de presiones $\left(\mathbf{E}_{b} \mathbf{P}_{b}\right)$ por la fuerza aerodinámica (ecuación (141)). La expresión, de forma compacta, se presenta a continuación:

$$
\mathbf{M}_{B_{b}}^{a, E_{b}}=\mathbf{E}_{b} \mathbf{P}_{b} \wedge \mathbf{F}_{b, B_{b}}^{a}
$$

El desarrollo de la ecuación resulta en las siguientes expresiones según las componentes:

$$
\begin{aligned}
M_{x_{B_{b}}}^{a, E_{b}}= & \frac{1}{2} \rho S y_{P_{b}}\left[\left(\left(\cos \theta_{b} \Omega y_{P_{b}}\right)^{2}+\left(-\cos \theta_{b} \Omega\left(e+x_{P_{b}}\right)-\sin \theta_{b} U_{W}\right)^{2}\right.\right. \\
& +\left(\sin \theta_{b} \Omega\left(e+x_{P_{b}}\right)-\frac{d \theta_{b}}{d t} y_{P_{b}}-\cos \theta_{b} U_{W}\right)^{2}\left(\sin \alpha_{b} C_{D}+\cos \alpha_{b} C_{L_{\alpha_{b}}}\right) \\
M_{y_{B_{b}}}^{a, E_{b}}= & \frac{1}{2} \rho S x_{P_{b}}\left[\left(\left(\cos \theta_{b} \Omega y_{P_{b}}\right)^{2}+\left(-\cos \theta_{b} \Omega\left(e+x_{P_{b}}\right)-\sin \theta_{b} U_{W}\right)^{2}\right.\right. \\
& +\left(\sin \theta_{b} \Omega\left(e+x_{P_{b}}\right)-\frac{d \theta_{b}}{d t} y_{P_{b}}-\cos \theta_{b} U_{W}\right)^{2}\left(\sin \alpha_{b} C_{D}+\cos \alpha_{b} C_{L_{\alpha_{b}}}\right)
\end{aligned}
$$




$$
\begin{aligned}
M_{z_{B_{b}}}^{a, E_{b}}= & \frac{1}{2} \rho S x_{P_{b}}\left[\left(\left(\cos \theta_{b} \Omega y_{P_{b}}\right)^{2}+\left(-\cos \theta_{b} \Omega\left(e+x_{P_{b}}\right)-\sin \theta_{b} U_{W}\right)^{2}\right.\right. \\
& +\left(\sin \theta_{b} \Omega\left(e+x_{P_{b}}\right)-\frac{d \theta_{b}}{d t} y_{P_{b}}-\cos \theta_{b} U_{W}\right)^{2}\left(\cos \alpha_{b} C_{D}+\sin \alpha_{b} C_{L_{\alpha_{b}}}\right)
\end{aligned}
$$

El conjunto de ecuaciones (141) y (143) son el punto de partida para el desarrollo en series de Taylor del apartado 3.11.1. En estas ecuaciones, aparecen las variables de estado y de control sobre las que interesa analizar la relevancia del orden de magnitud. Para completar el estudio, se definieron los siguientes parámetros adimensionales:

- $\mu_{z_{A}}=\frac{U_{0}}{\Omega R_{b}}$, relación de velocidades,

- $X_{P}=\frac{x_{P_{b}}}{R_{b}}$, posición adimensional del centro de presiones de la pala.

El cuadro siguiente muestra los órdenes de magnitud utilizados para el análisis en serie de Taylor:

\begin{tabular}{cc}
\hline \hline Parámetro & Orden de magnitud \\
\hline$\theta_{0}$ & 1 \\
$\theta_{1 S}$ & 1 \\
$\theta_{1 C}$ & 1 \\
$\mu_{z_{A}}$ & 1 o 2 \\
$e$ & 0 \\
$X_{P_{b}}$ & 1 \\
$y_{P_{b}}$ & 0 \\
\hline \hline
\end{tabular}

Cuadro 18: Órdenes de magnitud para los desarrollos en series de Taylor de fuerzas y momentos aerodinámicos.

Finalmente, el desarrollo en series de Taylor para las fuerzas y momentos aerodinámicos, resulta en las ecuaciones (81), (82) y (83).

\subsubsection{Obtención de términos gravitatorios}

Los efectos de los términos gravitatorios son presentados a continuación.

Por un lado, se representa el peso del cilindro del pararrotor en el sistema de referencia A: 


$$
\mathbf{F}_{H}^{g}=\left[\begin{array}{c}
-M_{H} \sin \Theta g \\
M_{H} \sin \Phi \cos \Theta g \\
M_{H} \cos \Phi \cos \Theta g
\end{array}\right]
$$

Las fuerzas gravitatorias que introduce la pala $b$, expresadas en el sistemad de referencia $A$, se muestran a continuación:

$$
\mathbf{F}_{b}^{g}=\left[\begin{array}{c}
-M_{b} \sin \Theta g \\
M_{b} \sin \Phi \cos \Theta g \\
M_{b} \cos \Phi \cos \Theta g
\end{array}\right]
$$

Los momentos gravitatorios de las dos palas respecto al centro de masas del pararrotor (punto $G$ ) se expresan del siguiente modo:

$$
\mathbf{M}_{A}^{g, G}=\left[\begin{array}{c}
M_{b} \cos \Theta g\left[\cos \psi\left(e+x_{G B_{b}}\right)-L \sin \Phi\right] \\
M_{b} g[-\cos \psi \cos \Phi \cos \Theta e-L \sin \Theta] \\
M_{b} g\left[(\cos \psi \sin \Phi \cos \Theta+\sin \Theta \sin \psi)\left(e+x_{G B_{b}}\right)\right]
\end{array}\right]
$$

Con estas expresiones se representa el efecto gravitatorio de cada componente del pararrotor en la ecuación canónica de la dinámica. 


\section{Referencias}

[1] W. Bollay, "A non-linear wing theory and its application to rectangular wings of small aspect ratio," ZAMM-Journal of Applied Mathematics and Mechanics/Zeitschrift für Angewandte Mathematik und Mechanik, vol. 19, no. 1, pp. 21-35, 1939.

[2] D. Kuchemann, "A simple method for calculating the span and chordwise loading on straight and swept wings of any given aspect ratio at subsonic speeds.," tech. rep., DTIC Document, 1952.

[3] B. Carmichael, "Low reynolds number airfoil survey, volume 1," 1981.

[4] T. Mueller, "Aerodynamic measurements at low reynolds numbers for fixed wing micro-air vehicles (development and operation of uavs for military and civil applications)(course held at the von kaman institute for fluid dynamics, belgium, 1999)," 2000.

[5] A. Pelletier and T. J. Mueller, "Low reynolds number aerodynamics of lowaspect-ratio, thin/flat/cambered-plate wings," Journal of Aircraft, vol. 37, no. 5, pp. 825-832, 2000.

[6] G. E. Torres and T. J. Mueller, "Low aspect ratio aerodynamics at low reynolds numbers," AIAA journal, vol. 42, no. 5, pp. 865-873, 2004.

[7] A. Rosen and D. Seter, "Vertical autorotation of a single-winged samara," Journal of applied mechanics, vol. 58, no. 4, pp. 1064-1071, 1991.

[8] D. Seter and A. Rosen, "Stability of the vertical autorotation of a single-winged samara," Journal of applied mechanics, vol. 59, no. 4, pp. 1000-1008, 1992.

[9] P. Crimi, "Analysis of samara-wing decelerator steady-state characteristics," Journal of Aircraft, vol. 25, no. 1, pp. 41-47, 1988.

[10] B. Roccia, S. Preidikman, L. Ceballos, and J. Massa, "Estudio de la aerodinámica no lineal e inestacionaria de semillas voladoras autorrotantes," Mecánica 
Comptacional Vol. XXXIII,Number 18. Multiphysics (B), pp. 2247-2266, 2014.

[11] K. Fregene, D. Sharp, C. Bolden, J. King, C. Stoneking, and S. Jameson, "Autonomous guidance and control of a biomimetic single-wing mav," in AUVSI Unmanned Systems Conference, pp. 1-12, Assoc. for Unmanned Vehicle Systems International Arlington, VA, 2011.

[12] S. Kang, J. Wang, and J. Shan, "Stability analysis of a visibility-reduced monocopter," Proceedings of the Institution of Mechanical Engineers, Part G: Journal of Aerospace Engineering, vol. 230, no. 4, pp. 653-667, 2016.

[13] D. Borgstrom, L. Paulsson, and L. Karlsen, "Aerodynamics of a rotating body descending from the separation position of an artillery munition shell," in 11 th Aerodynamic Decelerator Systems Technology Conference. San Diego, CA, US: AIAA, 1991.

[14] K.-F. Doherr and H. Schilling, "Nine-degree-of-freedom simulation of rotating parachute systems," Journal of Aircraft, vol. 29, no. 5, pp. 774-781, 1992.

[15] D. Levin and Z. Shpund, "Dynamic investigation of the angular motion of a rotating body-parachute system," Journal of aircraft, vol. 32, no. 1, pp. 93-99, 1995.

[16] D. Levin and Z. Shpund, "Canopy geometry effect on the aerodynamic behavior of cross-type parachutes," Journal of aircraft, vol. 34, no. 5, pp. 648-652, 1997.

[17] N. Peyada, A. Singhal, and A. Ghosh, "Trajectory modeling of a parafoil in motion using analytically derived stability derivative at high angle of attack," in 19th AIAA Aerodynamic Decelerator Systems Technology Conference and Seminar, p. 2511, 2007.

[18] A. Calise, D. Preston, and G. Ludwig, "Modeling for guidance and control design of autonomous guided parafoils," in 19th AIAA Aerodynamic Decelerator Systems Technology Conference and Seminar, p. 2560, 2007.

[19] W. PEPPER, "A new rotating parachute design having high performance," Journal of Spacecraft and Rockets, vol. 23, no. 2, pp. 222-224, 1986.

[20] V. J. Nadal Mora, Comportamiento aerodinámico de sondas atmosféricas en entornos aeroportuarios. PhD thesis, ETSI Aeronauticos, 2005.

[21] J. Piechocki, Estudio de la Dinámica del Vuelo de un Decelerador Aerodinámico Basado en el Concepto de Pararrotor. PhD thesis, Facultad de Ingeniería, UNLP, 2012.

[22] J. F. Martiarena, V. N. Mora, and J. Piechocki, "Experimental study of the 
effect of blade curvature and aspect ratio on the performance of a rotary-wing decelerator," Aerospace Science and Technology, vol. 43, pp. 471-477, 2015.

[23] S. Taamallah, X. Bombois, and P. M. Van den Hof, "Trajectory planning and trajectory tracking for a small-scale helicopter in autorotation," Control Engineering Practice, vol. 58, pp. 88-106, 2017.

[24] Q. Li, Y. Kamada, T. Maeda, J. Murata, K. Iida, and Y. Okumura, "Fundamental study on aerodynamic force of floating offshore wind turbine with cyclic pitch mechanism," Energy, vol. 99, pp. 20-31, 2016.

[25] M. Takao, Y. Kamada, Q. Li, et al., "Experimental investigation of the cyclic pitch control on a horizontal axis wind turbine in diagonal inflow wind condition," Energy, vol. 134, pp. 269-278, 2017.

[26] B. C. McKinney and G. C. Hudson, "Launch vehicle with engine mounted on a rotor," Dec. 1 1998. US Patent 5,842,665.

[27] Á. C. Tejero, Teoría de los helicópteros. Escuela Técnica Superior de Ingenieros Aeronáuticos, Universidad Politécnica de Madrid, 2009.

[28] J. Diebel, "Representing attitude: Euler angles, unit quaternions, and rotation vectors," Matrix, vol. 58, no. 15-16, pp. 1-35, 2006.

[29] J. Piechocki, V. N. Mora, and Á. Sanz-Andrés, "Numerical simulation of pararotor dynamics: Effect of mass displacement from blade plane," Aerospace Science and Technology, vol. 55, pp. 400-408, 2016.

[30] G. D. Padfield, Helicopter flight dynamics. John Wiley \& Sons, 2008.

[31] V. F. Zaitsev and A. D. Polyanin, Handbook of exact solutions for ordinary differential equations. CRC press, 2002.

[32] J. Figueroa, "Guía bip/iso para la expresión de la incertidumbre en las mediciones," Centro Nacional de Metrología. México, 1994.

[33] A. Roshko, "Experiments on the flow past a circular cylinder at very high reynolds number," Journal of Fluid Mechanics, vol. 10, no. 3, pp. 345-356, 1961.

[34] X. Ortiz, D. Rival, and D. Wood, "Forces and moments on flat plates of small aspect ratio with application to pv wind loads and small wind turbine blades," Energies, vol. 8, no. 4, pp. 2438-2453, 2015.

[35] D. Kuchemann, "A simple method for calculating the span and chordwise loading on straight and swept wings of any given aspect ratio at subsonic speeds.," tech. rep., DTIC Document, 1952. 
[36] T. Mueller, "Aerodynamic measurements at low reynolds numbers for fixed wing micro-air vehicles (development and operation of uavs for military and civil applications)(course held at the von kaman institute for fluid dynamics, belgium, 1999)," 2000.

[37] D. Levin and Z. Shpund, "Dynamic investigation of the angular motion of a rotating body-parachute system," Journal of aircraft, vol. 32, no. 1, pp. 93-99, 1995.

[38] J. G. Leishman, "Development of the autogiro: a technical perspective," Journal of Aircraft, vol. 41, no. 4, pp. 765-781, 2004.

[39] S. Houston and R. Brown, "Rotor-wake modeling for simulation of helicopter flight mechanics in autorotation," Journal of aircraft, vol. 40, no. 5, pp. 938-945, 2003.

[40] H. Y. Kim, D. J. Sheen, and S. O. Park, "Numerical simulation of autorotation in forward flight," Journal of aircraft, vol. 46, no. 5, pp. 1642-1648, 2009.

[41] C. Gallaway and W. Hankey, "Free-falling autorotating plate-a coupled fluid and flight mechanics problem," Journal of Aircraft, vol. 22, no. 11, pp. 983987, 1985.

[42] S. Houston, "Validation of a rotorcraft mathematical model for autogyro simulation," Journal of Aircraft, vol. 37, no. 3, pp. 403-409, 2000.

[43] A. R. Bramwell, D. Balmford, and G. Done, Bramwell's helicopter dynamics, ISBN 071313353 8. Elsevier, 2001.

[44] B. Obradovic, G. Ho, R. Barto, K. Fregene, and D. Sharp, "A multi-scale simulation methodology for the samarai monocopter uav," in AIAA Modeling and Simulation Technologies Conference, p. 5012, 2012.

[45] C.-T. Chen, Linear system theory and design, ISBN 019511777 8. Oxford University Press, Inc., 1995. 